\title{
ON THE RAMSEY PROPERTY OF FAMILIES OF GRAPHS
}

\author{
N. SAUER
}

\begin{abstract}
For graphs $A$ and $B$ the relation $A \rightarrow(B)_{r}^{1}$ means that for every $r$-coloring of the vertices of $A$ there is a monochromatic copy of $B$ in $A$. $\operatorname{Forb}\left(G_{1}, G_{2}, \ldots, G_{n}\right)$ is the family of graphs which do not embed any one of the graphs $G_{1}, G_{2}, \ldots, G_{n}$, a family $\mathscr{F}$ of graphs has the Ramsey property if for every graph $B \in \mathscr{F}$ there is a graph $A \in \mathscr{F}$ such that $A \rightarrow(B)_{r}^{1}$. Nešetřil and Rödl (1976) have proven that if either both graphs $G$ and $K$ are two-connected or the complements of both graphs $G$ and $K$ are two-connected then $\operatorname{Forb}(G, K)$ has the Ramsey property. We prove that if $\bar{G}$ is disconnected and $K$ is disconnected then $\operatorname{Forb}(G, K)$ does not have the Ramsey property, except for four pairs of graphs $(G, K)$.

A family $F$ of finite graphs is an age if there is a countable graph $G$ whose set of finite induced subgraphs is $\mathscr{F}$. We characterize those pairs of graphs $(G, H)$ for which $\operatorname{Forb}(G, H)$ is not an age but has the Ramsey property.
\end{abstract}

\subsection{INTRODUCTION}

In this paper we shall investigate the Ramsey properties of hereditary classes of finite graphs. For two graphs $A$ and $B$, and a positive integer $r$, let $A \rightarrow(B)_{r}^{1}$ mean that for every $r$-coloring of the vertices of $A$, there is a monochromatic copy of $B$ induced in $A$. We say that a class $\mathscr{F}$ of graphs has the Ramsey property (or is Ramsey, or has the partition property), if for every $B \in \mathscr{F}$, there exists $A \in \mathscr{F}$ so that $A \rightarrow(B)_{2}^{1}$. (It is a simple exercise to show that for any $r \in \omega$, if a class of graphs is a Ramsey class for two colors, it is a Ramsey class for $r$ colors.)

A class $\mathscr{F}$ of graphs is called hereditary if for any $G \in \mathscr{F}$, every induced subgraph $H$ of $G$ is also an element of $\mathscr{F}$. For any collection $\mathscr{G}$ of graphs, Forb $(\mathscr{G})$ is the set of all (finite) graphs none of which contains any element of $\mathscr{G}$ as an induced subgraph. It is easy to see that for any collection $\mathscr{G}$ of graphs, Forb $(\mathscr{G})$ is hereditary, and with a little more work, one can prove that any hereditary class $\mathscr{F}$ is $\operatorname{Forb}(\mathscr{G})$ for some collection $\mathscr{G}$ of graphs. (The set of finite graphs not in $\mathscr{F}$ will do for $\mathscr{G}$ and if one wishes to be a bit more economical the set of finite graphs not in $\mathscr{F}$ with a minimal number of vertices.)

For example, the class of all complete graphs is a hereditary class and forbids the graph consisting of two nonadjacent vertices.

Received by the editors June 3, 1991 and, in revised form, February 28, 1994.

1991 Mathematics Subject Classification. Primary 05C55; Secondary 05C15.

Key words and phrases. Graph-Ramsey theory, vertex coloring, excluded induced subgraphs.

This research has been supported by NSERC grant 69-1325. 
Problem 1. For which finite families $\mathscr{G}$ does Forb $(\mathscr{G})$ have the Ramsey property?

This problem in full generality seems to be very hard and the goal of this paper is to contribute to answering the problem when $\mathscr{G}$ consists of two graphs.

We may note here that, since induced graphs are used in these discussions, (dual) statements regarding partitions can be made in the complement, and thus one can show that $\operatorname{Forb}\left(G_{1}, G_{2}, \ldots, G_{n}\right)$ is Ramsey if and only if $\operatorname{Forb}\left(\overline{G_{1}}, \overline{G_{2}}, \ldots, \overline{G_{n}}\right)$ is Ramsey. In [1], the following has been shown.

Theorem 1. If $G_{1}, G_{2}, \ldots, G_{n}$ are all two-connected (or all the complements $\overline{G_{1}}, \overline{G_{2}}, \ldots, \overline{G_{n}}$ are two-connected) then $\operatorname{Forb}\left(G_{1}, G_{2}, \ldots, G_{n}\right)$ is Ramsey.

In the case of a single forbidden graph, if either $G$ or $\bar{G}$ is two-connected, then $\operatorname{Forb}(G)$ is Ramsey. Those remaining graphs (which are neither twoconnected, nor are their complements) have been identified in [4], and results (see [4], [5], [6]) have been obtained regarding whether or not $\operatorname{Forb}(G)$ is then Ramsey. For example, both Forb $\left(P_{2}\right)$ and Forb $\left(P_{3}\right)$ are Ramsey, however, for a large portion (in some sense, almost all) of the remaining cases, $\operatorname{Forb}(G)$ is not Ramsey.

Most of the cases where $\operatorname{Forb}(G)$ is not Ramsey were proved using concepts of amalgamation (see next section). These concepts will again play a role when examining $\operatorname{Forb}(G, H)$, the main topic of this paper; we define one of them now.

An embedding of a graph $G$ into a graph $H$ is an injective map $\phi: V(A) \rightarrow$ $V(B)$ so that $(\phi(x), \phi(y))$ is an edge in $H$ if and only if $(x, y)$ is an edge in $G$. We say that a class of graphs $\mathscr{F}$ has the joint-embedding property (or is updirected) if for any $A$ and $B$ in $\mathscr{F}$, there exists a $C \in \mathscr{F}$ and embeddings $\alpha: A \rightarrow C, \beta: B \rightarrow C$.

It turns out [4] that the classes $\operatorname{Forb}(G)$ are updirected, however this is no longer (necessarily) the case for classes of the form $\operatorname{Forb}(G, H)$ (where neither of $G$ or $H$ embeds the other). The main result of this paper completely classifies the Ramsey classes for which $\operatorname{Forb}(G, H)$ is not updirected. (The case where $\operatorname{Forb}(G, H)$ is updirected is still open.)

Theorem 2. A family $\operatorname{Forb}(G, H)$ of graphs which is not updirected has the Ramsey property if and only if $(G, H)$ is one of the following three pairs:

(a) $G=P_{2}, H$ is the disjoint union of an edge and a vertex

$$
\text { ( } G=\text { qo }, H=\xi \circ) \text {. }
$$

(b) $G$ is a triangle with a pendant edge attached, $H$ is the disjoint union of a triangle and a vertex

$$
\left(G=\sigma_{0}, H=\sigma_{0}\right) \text {. }
$$

(c) $G=K_{1,3}, H$ is the disjoint union of $P_{2}$ and a vertex.

$$
\left(G=9 g, H=9 \rho_{0}\right) \text {. }
$$

Of the pairs $(G, H)$ not covered by Theorem 3, we will need only examine those for which $\bar{G}$ and $H$ are both disconnected by the following.

Lemma 1. If $\operatorname{Forb}(G, H)$ is not updirected, then either both $G$ and $\bar{H}$ are disconnected, or both $\bar{G}$ and $H$ are disconnected. 
The proof of Lemma 1 is not complicated, however we defer it to a later section. Of the pairs $(G, H)$ described in Lemma 1 , we completely determine those for which $\operatorname{Forb}(G, H)$ has the partition property; there are four such pairs.

Theorem 3. If $G$ and $H$ are graphs neither embeddable into the other, with $\bar{G}$ and $H$ disconnected, then $\operatorname{Forb}(G, H)$ has the Ramsey property if and only if $(G, H)$ is one of the following pairs:

(a) $G=P_{2}, H$ is the disjoint union of an edge and a vertex.

(b) $G$ is a triangle with a pendant edge attached, $H$ is the disjoint union of $a$ triangle and a vertex.

(c) $G=K_{1}, 3, H$ is the disjoint union of $P_{2}$ and a vertex.

(d) $G=K_{2}, H$ is the disjoint union of two vertices.

Since the class forbidding the pair in (d) of Theorem 4 is the trivial family consisting of one vertex (trivially seen to be updirected), Theorem 2 is a consequence of Theorem 3; hence we need only prove Theorem 3 . This proof is quite detailed and comprises most of this paper.

\subsection{Amalgamation and Ramsey Classes}

We say that a class of graphs $\mathscr{F}$ has the amalgamation property if for any $A$ and $B$ in $\mathscr{F}$, and any $a \in V(A), b \in V(B)$, there exists a $C \in \mathscr{F}$ and embeddings $\alpha: A \rightarrow C, \beta: B \rightarrow C$ such that $\alpha(a)=\beta(b)$. [There may or may not be edges between the remainder.] If furthermore, $\alpha$ and $\beta$ exist so that $(\alpha(V(A)) \backslash\{a\}) \cup(\beta(V(B)) \backslash\{b\})=\varnothing$, then we say that $\mathscr{F}$ has disjoint amalgamation (this might be more appropriately thought of as singlepoint amalgamation).

A rather interesting relationship [4], [15] between these properties is the following:

Theorem 4. If a hereditary family $\mathscr{F}$ which is updirected is also Ramsey, then it has the disjoint amalgamation property (and hence the amalgamation property).

It was also shown in [1] that Ramsey classes have the amalgamation property. Theorem 4 was the central tool in characterizing when Forb $(G)$ was Ramsey by showing when the family did not have disjoint amalgamation. We will need a slight modification of Theorem 4 whose proof is actually contained in the proof of Theorem 4 but not in the statement. H. Kierstead and V. Rödl gave a much better proof in an unpublished manuscript. The Kierstead, Rödl proof can be found in [15] and we will use Lemma 3 of [15] which we state as

Theorem 5. Let $\mathscr{F}$ be a family of graphs, $A \in \mathscr{F}$ with $a, b \in V(A)$ and $r=2 k-1$. If there is a graph $B \in \mathscr{F}$ with $B \rightarrow(A)_{r}^{1}$ then the graph $A$ can be disjointly amalgamated with $A$ on $a \simeq b$ into a graph $C \in \mathscr{F}$.

If $\mathscr{F}$ is a set of finite graphs with $B \in \mathscr{F}$ we say that $\mathscr{F}$ is chromatically unbounded for the graph $B$ if for every $r \in \omega$ there is a graph $A \in \mathscr{F}$ such that $A \rightarrow(B)_{r}^{1}$ (negation chromatically bounded). If $\mathscr{F}$ is chromatically unbounded for the edge $K_{2}$ then $\mathscr{F}$ is chromatically unbounded. If $\mathscr{F}=\operatorname{Forb}(\mathscr{G})$ for some graph $G$ we denote by $\mathscr{N}=\mathscr{N}_{G}$ the set of chromatically bounded graphs in $\operatorname{Forb}(G)$. With these notions we obtain the following corollary to Theorem 5. 
Corollary. If the graph $X$ is chromatically unbounded in the hereditary family $\mathscr{F}$ of graphs and if $X$ embeds the graphs $L$ and $R$ then $L$ can be disjointly amalgamated with $R$ on any pair of vertices into a graph $C \in \mathscr{F}$. In particular if $G$ is a graph and the graph $X \notin \mathscr{N}_{g}$ embeds the graphs $L$ and $R$ then $L$ can be disjointly amalgamated with $R$ on any pair of vertices into a graph $C \in \mathscr{F}$.

A set of distinct graphs is called an age if it is precisely the collection of copies of finite induced subgraphs of some (at most) countable graph. It is not difficult to show (see [8]) that a family of graphs is an age if it is hereditary and has the joint-embedding property. So Theorem 2 says that a family $\operatorname{Forb}(G, H)$ which is not an age has the Ramsey property in only three cases. The cases where $\operatorname{Forb}(G, H)$ is an age are not addressed in this paper.

Let $\mathfrak{M}$. be the set of all finite graphs $G$ which contain a cutpoint $u$ which is adjacent to all other vertices of $G$. Theorem 5.1 of [4] states that

Theorem 6. For every graph $G=\mathfrak{M}-P_{2}$ the family $\operatorname{Forb}(G)$ does not have the Ramsey property.

\subsection{Chromatic NUMBERS AND RAMSEY CLASSES}

A hypergraph $H$ is a set $E(H)$ of subsets of the set $V(H)$, the vertices of $H$. An m-uniform hypergraph $H$ is a set $E(H)$ of subsets of size $m$ of the set $V(H)$, the vertices of $H$. The set $E(H)$ is the set of edges of $H$. A graph is a 2-uniform hypergraph.

The chromatic number of a hypergraph $H$ is the smallest number of blocks into which $V(H)$ can be partitioned so that no edges of $H$ is a subset of one of the blocks. A cycle of length $n$ of the hypergraph $H$ is a sequence $E_{0}, E_{1}, \ldots, E_{n-1}$ of edges of $H$ and a sequence $x_{0}, x_{1}, \ldots, x_{n_{1}}$ of vertices of $H$ such that for each $i \in n, x_{i} \in E_{i} \cap E_{i+1}$. The girth of $H$ is the smallest number $g$ such that $H$ contains a cycle of length $g$. Note that if two edges of $H$ have at least two vertices in common they form a cycle of length two. The hypergraph $H$ is a forest if it does not contain any circuits.

We will use the theorem of Erdös and Hajnal [7], which says that for every triple of numbers $k, g, r$ there exists a $k$-uniform hypergraph $H$ with girth at least $g$ and chromatic number at least $r$.

If $A$ and $B$ are two graphs let hypergraph $(A, B)$ denote the hypergraph whose vertex set is the set of vertices of $A$ and whose edges are the vertex sets of copies of $B$ which are induced subgraphs of $A$. Clearly, if $A \rightarrow(B)_{r}^{1}$ holds, then the chromatic number of hypergraph $(A, B)$ is larger than $r$. The chromatic number of hypergraph $\left(A, K_{2}\right)$ is the chromatic number of $A$. Let $B$ be a graph, $H$ a $|V(B)|$-uniform hypergraph which does not contain any cycles of length two and $A$ a graph which we construct from the hypergraph $H$ by putting a copy of $B$ into every hyperedge of $H$. We will say that $A$ has been constructed from $H$ by inserting copies of $B$ into the hyperedges of $H$. Observe that if the chromatic number of $H$ is larger than $r$, then $A \rightarrow(B)_{r}^{1}$ holds. This then can be extended as follows: If $\left\{G_{0}, G_{1}, \ldots, G_{n-1}\right\}$ is a set of graphs such that for each $i \in n$ there is a two-connected subgraph of $G_{i}$ which cannot be embedded into the graph $B$, the chromatic number of $H$ is larger than $r$ and the girth of $H$ is sufficiently large then $A \in \operatorname{Forb}\left(G_{0}, G_{1}, \ldots, G_{n-1}\right)$ and 
$A \rightarrow(B)_{r}^{1}$. Such a graph $H$ exists by [7]. If all of the graphs $G_{i}$ are twoconnected then we obtain the Nešetřil and Rödl observation [1], which we have stated as Theorem 1.

Note that the set $\mathscr{F}$ of graphs has the Ramsey property if and only if it is chromatically unbounded for every graph $B \in \mathscr{F}$. In order to characterise Ramsey families of graphs it is therefore of interest to characterise for a given graph $B$ the sets $\mathscr{F}$ of graphs which are chromatically unbounded for $B$. Unfortunately even for $B=K_{2}$ this is a very difficult problem as we will explain in the next paragraph.

If no graph in a set $\left\{G_{1}, G_{2}, \ldots, G_{n}\right\}$ of graphs contains a complete graph then the set $\operatorname{Forb}\left(G_{1}, G_{2}, \ldots, G_{n}\right)$ contains arbitrary large complete graphs and hence is chromatically unbounded. On the other hand it follows from the fact that there are graphs of arbitrarily large chromatic number and arbitrarily large girth that if every graph in the set $\left\{G_{1}, G_{2}, \ldots, G_{n}\right\}$ of graphs contains a cycle, then the family $\operatorname{Forb}\left(G_{1}, G_{2}, \ldots, G_{n}\right)$ of graphs is chromatically unbounded. Hence, if a family $\operatorname{Forb}(G, H)$ of graphs is chromatically bounded then one of the two graphs $G$ and $H$, say $G$, must be a complete graph and the other one a forest. In this context the following conjecture of Gyárfás [10] and independently Sumner [11] is of interest: For every tree $T$ and number $n$ there is a number $r$ such that the chromatic number of every graph for $\operatorname{Forb}\left(T, K_{n}\right)$ is smaller than $r$. Note that to every forest $B$ there exists a tree $T$ such that $B$ is an induced subgraph of $T$. Hence, if the conjecture of Gyárfás and Sumner is correct and $B$ is a forest, then $\operatorname{Forb}\left(B, K_{n}\right)$ is chromatically bounded. The conjecture of Gyárfás and Sumner raises a very difficult problem. Even limited results require often quite ingenious methods. See [12], [13] and [14].

\subsection{NotATION USED IN PROOFS}

We only consider finite, undirected, simple graphs. If $G$ is a graph, $V(G)$ denotes the set of vertices of $G$ and $E(G)$ the set of edges of $G$. The edges of $G$ are two-element subsets of $V(G)$. If $H$ and $L$ are two subgraphs of $G$, then $E(H, L)$ is the set of edges of $G$ which contains at least one vertex of $H$ and at least one vertex of $L$. If $S \subset V(G), G \mid S$ will denote the graph $H$ with $S=V(H)$ in which the vertex $a$ is adjacent to the vertex $b$ if and only if $a$ is adjacent to $b$ in $G$. Such a graph $H$ is called an induced subgraph of $G$, it is induced by $S$. For $a \in V(G), G-a$ is the graph $G \mid(V(G)-a)$ and more generally, if $S \subset V(G), G-S$ is the graph $G \mid(V(G)-S)$. If $H$ is a subgraph of $G$ we will write $G-H$ instead of $G-V(H) . \bar{G}$ denotes the complement of $G$ and if $\mathscr{F}$ is a family of graphs then $\bar{F}=\{\bar{G}: G \in \mathscr{F}\}$. The family $\bar{F}$ will be called the dual family to the family of graphs $\mathscr{F}$. An injection $\alpha: V(G) \rightarrow V(H)$ is an embedding from the graph $G$ to the graph $H$ if for all vertices $a, b \in V(G),\{a, b\}$ is an edge of $G$ if and only if $\{\alpha(a), \alpha(b)\}$ is an edge of $H$. For two graphs $A$ and $G$ we say that the graph $A$ does not embed the graph $G$ if there is no embedding from $G$ into $A$. We will write $G \cong H$ to indicate that the graphs $G$ and $H$ are isomorphic (there is then an embedding $\alpha$ from $G$ to $H$ with $\alpha(V(G))=V(H))$.

If $G_{1}, G_{2}, \ldots, G_{n}$ are graphs then $\operatorname{Forb}\left(G_{1}, G_{2}, \ldots, G_{n}\right)$ is the family of graphs which do not embed any one of the graphs $G_{1}, G_{2}, \ldots, G_{n}$. Note that if the graph $G$ can be embedded into the graph $K$, then $\operatorname{Forb}(G, K)=\operatorname{Forb}(G)$. 
We will investigate families $\operatorname{Forb}(G, K)$ of graphs and assume that none of the graphs $G$ and $K$ can be embedded into the other. We will denote by $P_{n}$ the path with $n$ edges and by $K_{n}$ the complete graph on $n$ vertices. The empty graph is the graph with no vertices.

The theory of graphs with embeddings as morphisms has complementation of graphs as a natural dual theory. We will make use of this duality and speak of dual statements and definitions. So for example, the dual statement to "the vertices $a$ and $b$ are adjacent" is the statement "the vertices $a$ and $b$ are not adjacent". Let $G$ and $H$ be two graphs; then the graph $G+H$ is the disjoint union of (copies of) $G$ and $H$. We will say that $G$ and $H$ are components of $G+H$. If $G_{i}$ for $i \in s$ is a sequence of graphs, then $\sum_{i \in s+1} G_{i}=\sum_{i \in s} G_{i}+G_{s}$. The graph $G \oplus H$ is the complement of the graph $\bar{G}+\bar{H}$. That is, $G \oplus H$ is the disjoint union of $G$ and $H$ with all edges in between. Clearly $G \oplus H$ is the dual definition to the definition of $G+H$. A graph $L$ is disconnected if there are two nonempty graphs $G$ and $H$ such that $L=G+H$. The graph $L$ is connected if it is not disconnected. The subgraph $L_{1}$ of $L$ is a connected component of $L$ if $L_{1}$ is connected, not the empty graph and there is a graph $L_{2}$ such that $L=L_{1}+L_{2}$. For $1 \leq c \in \omega, L$ is $c$-connected, if for every set $S \in V(L)$ with $|S|<c, L-S$ is connected. Observe that the graph $L$ is 1 -connected just in case it is connected.

A block of $L$ is a maximal 2-connected induced subgraph of $L$ [2], [3]. Note that every two blocks of $L$ have at most one vertex in common and that every edge of $L$ is in exactly one block of $L$. Also, in a graph, there is no cycle of blocks. That is, the hypergraph of blocks does not contain a cycle. A dual block is the dual notion to the notion of a block. That means, a dual block of $L$ is the complement of a block of $\bar{L}$. A dual component of $L$ is the complement of a connected component of $\bar{L}$. The graph $X$ is a $Y$-tree if the set $\mathfrak{S}=\{S \subseteq V(X): X \mid S$ is a dual block of $X\}$ has the property that for every $S \in \mathfrak{S}$ there is an embedding from $X \mid S$ into $Y$.

\subsection{Preliminary Results}

Lemma 1. If $\operatorname{Forb}(G, H)$ is not updirected, then either both $G$ and $\bar{H}$ are disconnected, or both $\bar{G}$ and $H$ are disconnected.

Proof. Assume that the graphs $A \in \operatorname{Forb}(G, H)$ and $B \in \operatorname{Forb}(G, H)$ are witness to $\operatorname{Forb}(G, H)$ is not updirected. Then neither $A+B \in \operatorname{Forb}(G, H)$ nor $A \oplus B \in \operatorname{Forb}(G, H)$. This implies that one of the graphs $G$ and $H$, say $G$, can be embedded into $A+B$ but not into $A$ and not into $B$. Hence $G$ is disconnected.

Because $G$ cannot be embedded into $A$ or $B$ and $G$ is disconnected it cannot be embedded into $A \oplus B$. Hence $H$ can be embedded into $A \oplus B$. Because $H$ cannot be embedded into $A$ or $B$ it follows that $\bar{H}$ is disconnected.

One of the main tools used to establish Theorem 3 is the following observation:

Lemma 2. Let $\mathscr{F}$ be a nontrivial family of graphs which has the Ramsey property and for all $i \in n \in \omega$ let $A_{i} \in \mathscr{F}$. If there is a graph $J \in \mathscr{F}$ such that for all $i \in n, A_{i}$ can be embedded into $J$, then $A_{0}+A_{1}+\cdots+A_{n-1} \in \mathscr{F}$ or $A_{0} \oplus A_{1} \oplus \cdots \oplus A_{n-1} \in \mathscr{F}$. 
Proof. We prove first that if $J \in \mathscr{F}$ then $J+J \in \mathscr{F}$ or $J \oplus J \in \mathscr{F}$. If $|V(J)|=1$ then $J+J \in \mathscr{F}$ or $J \oplus J \in \mathscr{F}$ because $\mathscr{F}$ is not the trivial family and hence contains a graph with at least two vertices. We assume then that $|V(J)|>1$. Choose a graph $G \in \mathscr{F}$ such that $G \rightarrow(J)_{2}^{1}$, put $s=2^{|V(G)|}+2$ and $r=2^{|V(G)|}$. Let $H \in \mathscr{F}$ be such that $H \rightarrow(G)_{s}^{1}$ and let $G_{1}$ be a fixed copy of $G$ in $H$. Note that $H-G_{1} \rightarrow(G)_{r}^{1}$ because $G$ is not the singleton graph. If for some set $S \subseteq V\left(G_{1}\right)$ the graph $H \mid S$ does not contain a copy of $J$ then the graph $H \mid\left(V\left(G_{1}\right)-S\right)$ contains a copy $J_{1}$ of $J$ because $G \rightarrow(J)_{2}^{1}$. We associate now with each vertex $a \in V\left(H-G_{1}\right)$ the set $\gamma(a)$ of vertices in $V\left(G_{1}\right)$ which are adjacent to $a$. The kernel of $\gamma$ partitions $H-G_{1}$ into $r$ classes and hence there is a set $S \subseteq V\left(G_{1}\right)$ such that $\gamma^{-1}(S)$ contains a copy $G_{2}$ of $G$. If $H \mid S$ contains a copy of $J$ then because $G_{2}$ contains a copy of $J, H$ contains a copy of $J \oplus J$. If $H \mid S$ does not contain a copy of $J$ then $H \mid\left(V\left(G_{1}\right)-S\right)$ contains a copy of $J$ and hence, because $G_{2}$ contains a copy of $J, H$ contains a copy of $J+J$.

If $R \in \mathscr{F}$ we denote by $R \uplus R$ the graph $R+R \in \mathscr{F}$ and the graph $R \oplus R$ otherwise. Also $\amalg_{0} R=R$ and $\amalg_{m} R=\left(\amalg_{m-1} R\right) \uplus\left(\amalg_{m-1} R\right)$. Using this notation it follows that either $A_{0}+A_{1}+\cdots+A_{n-1}$ or $A_{0} \oplus A_{1} \oplus \cdots \oplus A_{n-1}$ can be embedded into $\amalg_{2 n} J$.

Lemma 3. If $G$ and $H$ are one of the following three pairs of graphs then $\operatorname{Forb}(G, H)$ has the Ramsey property but not the joint-embedding property. $G \cong P_{2}$ and $H \cong \bar{P}_{2}$ or $G \cong K_{1} \oplus\left(K_{1}+K_{2}\right)$ and $H \cong K_{1}+K_{3}$ or $G \cong$ $K_{1} \oplus\left(K_{1}+K_{1}+K_{1}\right)$ and $H \cong K_{1}+P_{2}$.

Proof. Case 1: $G \cong P_{2}$ and $H \cong \bar{P}_{2}$. Observe that if the graph $G \cong P_{2}$ cannot be embedded into a graph $R$, then the adjacency relation on $R$ is transitive. Hence $R$ is the sum (+) of complete graphs. A graph which is the sum of complete graphs and into which the graph $H \cong \bar{P}_{2}$ cannot be embedded is either a complete graph or the complement of a complete graph. Clearly then $\operatorname{Forb}(G, H)$ has the Ramsey property but not the joint-embedding property.

Case 2: $G \cong K_{1} \oplus\left(K_{1}+K_{2}\right)$ and $H \cong K_{1}+K_{3}$. Note that every triangle free graph is an element of $\operatorname{Forb}(G, H)$. We will argue that if $R \in \operatorname{Forb}(G, H)$ and $R$ contains a triangle, then $R$ is a complete $m$-partite graph for some $m \geq 3$. So, assume that $R \in \operatorname{Forb}(G, H)$ and that $R$ contains a triangle. Let $C$ be the largest complete subgraph of $R$ with $|V(C)|=m \geq 3$. If $x \in V(R-C)$ then, because $H$ cannot be embedded into $R, x$ is adjacent to some vertex of $C$. Because $G$ cannot be embedded into $R, x$ is adjacent to at least $m-1$ vertices of $C$. Because $C$ is a maximal complete subgraph of $R, x$ is adjacent to exactly $m-1$ vertices of $C$. No two vertices of $R$ which are adjacent to the same subset of vertices of $C$ can be adjacent, otherwise $C$ would not be maximal. Every vertex $x \in V(C)$ induces, together with those vertices of $C$ which are adjacent to $x$, a complete graph $C_{x}$ on $m$ vertices. Every vertex $y \in V\left(R-C_{x}\right)$ is adjacent with exactly $m-1$ vertices of $C_{x}$. Hence if for some two vertices $x, y \in V(R), C_{x} \neq C_{y}$, then $x$ and $y$ are adjacent. We conclude that $R$ is an $m$-partite graph in which the vertices $x$ and $y$ are in the same part if and only if $C_{x}=C_{y}$.

It follows that $\operatorname{Forb}(G, H)$ has the Ramsey property because the family of triangle free graphs has the Ramsey property and the family of complete $m$-partite graphs for $m \geq 3$ has the Ramsey property. There is no graph 
$J \in \operatorname{Forb}(G, H)$ such that both graphs $K_{3}$ and $K_{2}+K_{1}$ can be embedded into $J$, because $K_{2}+K_{1}$ cannot be embedded into any complete $m$-partite graph. Hence $\operatorname{Forb}(G, H)$ does not have the joint embedding property.

Case 3: $G \cong K_{1} \oplus\left(K_{1}+K_{1}+K_{1}\right)$ and $H \cong K_{1}+P_{2}$. The complement of the graph $G$ is the graph $K_{1}+K_{3}$ which is the graph $H$ from Case 2 and the complement of the graph $H$ is the graph $K_{1} \oplus\left(K_{1}+K_{2}\right)$ which is the graph $G$ from Case 2. Hence Case 3 is the complementary situation to Case 2.

Lemma 4. If $X, Y, Z$ are finite graphs which have pairwise no vertex in common and $\alpha$ is an embedding from $X+Y$ into $X+Z$ then there exists an embedding $\beta$ from $Y$ into $Z$. Also, if $X, Y, Z$ are finite graphs and $\alpha$ is an embedding from $X \oplus Y$ into $X \oplus Z$ then there exists an embedding $\beta$ from $Y$ into $Z$.

Proof. As the second assertion is the dual statement to the first, it suffices to prove the first assertion. For every vertex $y \in V(Y)$ there exists a power $p_{y}$ such that $\alpha^{p_{y}}(y) \in V(Z)$ because $\alpha$ is one to one and the graphs are finite. Let for every vertex $y \in V(Y), \beta(y)=\alpha^{p_{y}}(y)$. Vertices in the same connected component of $Y$ have the same power because adjacent vertices have the same power. Hence if the vertices $y_{1}$ and $y_{2}$ of $Y$ are adjacent, then $\beta\left(y_{1}\right)$ and $\beta\left(y_{2}\right)$ are also adjacent. If the vertices $y_{1}$ and $y_{2}$ of $Y$ are different, then $\beta\left(y_{1}\right)$ and $\beta\left(y_{2}\right)$ are different. Otherwise, consider the smallest two numbers $a$ and $b$ such that $\alpha^{a}\left(y_{1}\right)=\alpha^{b}\left(y_{2}\right)$. If both numbers $a$ and $b$ are not zero, then $\alpha^{a-1}\left(y_{1}\right) \neq \alpha^{b-1}\left(y_{2}\right)$ which is not possible because $\alpha$ is injective. But none of the two numbers $a, b$ can be zero, because no vertex of $Y$ is in the image of $\alpha$. Applying the above considerations to $\alpha^{-1}$ we conclude that if the vertices $y_{1}$ and $y_{2}$ of $Y$ are not adjacent, then $\beta\left(y_{1}\right)$ and $\beta\left(y_{2}\right)$ are also not adjacent. Hence $\beta$ is an embedding from $Y$ into $Z$.

Let $M$ be a nonempty graph and $H$ a $|V(M)|$-uniform hypergraph. With each hyperedge $E$ of $H$ we associate a one to one function $\varphi_{E}$ from $E$ onto $V(M)$. Let $L_{1}$ be the graph with vertex set $V\left(L_{1}\right)=V(H)$. Two vertices $a$, $b \in V\left(L_{1}\right)$ are not adjacent if and only if there is a hyperedge $E$ of $H$ such that $a, b \in E$ and $\varphi_{E}(a)$ is not adjacent to $\varphi_{E}(b)$ in $M$. We will say that the graph $L_{1}$ has been constructed from the hypergraph $H$ by embedding $M$ into the edges of $H$. Note that if the chromatic number of $H$ is larger than $r$, then $L_{1} \rightarrow(M)_{r}^{1}$.

Lemma 5. Let $M$ be a nonempty graph, $T$ a dual component of $M$ with a maximal number of vertices, $H$ a $|V(M)|$-uniform hypergraph with girth at least five and $L_{1}$ a graph constructed from the hypergraph $H$ by embedding $M$ into the edges of $H$. Let $z_{0}$ and $v$ be two different new vertices with $z_{0}$, $v \notin V(H) \cup V(M)$. Denote by $L$ the graph $L=z_{0} \oplus L_{1}$. Then $L \in \operatorname{Forb}(T+v)$. (It follows then that $L \in \operatorname{Forb}(M+v)$. Girth five is not really necessary here but will be needed later.)

Proof. Assume that there is an embedding $\alpha$ from $T+v$ into $L$. The vertex $v$ is in $T+v$ not adjacent to any vertex of $T$. Hence $\alpha(v) \neq z_{0}$, and $z_{0} \notin$ $V(\alpha(T))$. Observe that to every vertex $a \in V(T)$ there is an edge $E_{a}$ of $H$ which contains both vertices $\alpha(v)$ and $\alpha(a)$. Because the girth of $H$ is larger than two, there is only one such edge $E_{a}$ for every vertex $a \in V(T)$. If $a$, $b \in V(T)$ are two vertices for which $E_{a} \neq E_{b}$, then $\alpha(a)$ and $\alpha(b)$ are adjacent 
in $L$ because $H$ does not contain a cycle of length three. The vertices $a$ and $b$ are then adjacent in $T$. This then means that $T \mid \alpha\left(E_{a}\right)$ is a dual component of $T$ in contradiction to $\bar{T}$ being connected. Hence there is some edge $E$ of $H$ such that $V(\alpha(T)) \cup\{\alpha(v)\} \subseteq E$. There is an embedding $\varphi_{E}$ from $L \mid E$ to $M$. Observe that the complement of the graph $L \mid(V(\alpha(T)) \cup\{\alpha(v)\})$ is connected. Hence the complement of the graph $\varphi_{E}(L \mid(V(\alpha(T)) \cup\{\alpha(v)\}))$ is connected in contradiction to the choice of $T$ as a largest dual component of $M$.

Let $Q$ be a connected graph with a vertex $z \in V(Q)$. We say a subset $S \subseteq V(Q)$ is attached at the vertex $z$ (or briefly, attached) if $z \in S$ and $Q \mid S$ is connected. Note that the union of two attached subsets is again an attached subset and that $\{z\}$ and $V(Q)$ are attached subsets of $Q$. Let $\Xi_{z}(Q)$ be the number of attached subsets of $V(Q)$.

Lemma 6. Let $M$ be a nonempty graph and $u, v$ two vertices not in $M$. Put $D=v+M$, and $G=u \oplus D$ and let $Q \in \operatorname{Forb}(G)$ be a connected graph such that there is an embedding $\alpha$ from $D$ into $Q$ and $z$ a vertex in $V(Q)$. Let $H$ be $a|V(M)|$-uniform hypergraph of girth $g$ larger than or equal to five and chromatic number $\chi(H)>r=\Xi_{z}(Q)$. Assume that the graph $L_{1}$ has been constructed from the hypergraph $H$ by embedding $M$ into the edges of $H$ and that $L=z_{0} \oplus L_{1}$ for some vertex $z_{0}$ not in $L_{1}$ or $G$. Then, no graph $J \in \operatorname{Forb}(G)$ amalgamates $L$ and $Q$ disjointly on $z_{0} \simeq z$.

Proof. Assume to the contrary that there is such a graph $J \in \operatorname{Forb}(G)$ which amalgamates $L$ and $Q$ disjointly on $z_{0} \simeq z$. Without loss of generality we may assume that $L$ and $Q$ actually are induced subgraphs of $J$ and that $V(L) \cup V(Q)=V(J)$. For $a \in V(H)$ denote by $\Gamma(a)$ the maximal attached subset of $Q$ all of whose vertices are in $J$ adjacent to $a$. Because $a$ is adjacent to $z_{0} \simeq z$ and the union of two attached subsets is again an attached subset $\Gamma(a)$ is well defined for every $a \in V(H)$. The function $\Gamma$ induces an $r$-colouring of the vertices of $V(H)$. Note that if $S$ is an attached subset of $Q$ with $S \neq V(Q)$, then $\Gamma^{-1}(S)$ does not contain any hyperedge $E$ of $H$. Otherwise, if $b \in V(Q)-S$ and $b$ is adjacent to some vertex $c$ in $S, J \mid(E \cup\{c\} \cup\{b\})$ is an isomorphic copy of $G$. If there is no $a \in V(H)$ with $\Gamma(a)=V(Q), \Gamma$ would be a good $r-1$ colouring of $H$, contrary to the definition of $H$. This leads us to the desired contradiction because if for some vertex $a \in V(H)$, $\Gamma(a)=V(Q)$, then $J \mid(V(\alpha(D)) \cup\{a\})$ is an isomorphic copy of $G$.

\subsection{Proof of THEOREMS}

We are now in the position to prove the main theorem subject to the lemmas proven in the next chapters. The statements and proofs of some of those lemmas is technical. The present section shows how those lemmas are being used to establish the main results.

Theorem 3. If $G$ and $H$ are graphs neither embeddable into the other, with $\bar{G}$ and $H$ disconnected, then $\operatorname{Forb}(G, H)$ has the Ramsey property if and only if $(G, H)$ is one of the following pairs:

(a) $G=P_{2}, H$ is the disjoint union of an edge and a vertex. $\left(H=\overline{P_{2}}\right.$. $)$

(b) $G$ is a triangle with a pendant edge attached, $H$ is the disjoint union of a triangle and a vertex. $\left(G=K_{1} \oplus\left(K_{1}+K_{2}\right), H=K_{1}+K_{3}\right.$.) 
(c) $G=K_{1,3}, H$ is the disjoint union of $P_{2}$ and a vertex. ( $G=K_{1} \oplus\left(K_{1}+\right.$ $\left.K_{1}+K_{1}\right), H=K_{1}+P_{2}$.)

(d) $G=K_{2}, H$ is the disjoint union of two vertices. $\left(H=K_{1}+K_{1}\right.$.)

Proof. Let $\mathscr{E}_{1}=\operatorname{Forb}\left(P_{2}, \overline{P_{2}}\right), \mathscr{E}_{2}=\operatorname{Forb}\left(K_{1} \oplus\left(K_{1}+K_{2}\right), K_{1}+K_{3}\right), \mathscr{E}_{3}=$ $\operatorname{Forb}\left(K_{1} \oplus\left(K_{1}+K_{1}+K_{1}\right), K_{1}+P_{2}\right)$ and $\mathscr{E}_{4}=\operatorname{Forb}\left(K_{2}, K_{1}+K_{1}\right)$. Note that the family $\mathscr{E}_{1}$ and the trivial family $\mathscr{E}_{4}$ are self dual while the family $\mathscr{E}_{3}$ is dual to the family $\mathscr{E}_{2}$. It follows from Lemma 3 and the observation that the trivial family has the Ramsey property, that in each of the exceptional four cases, $\operatorname{Forb}(G, H)$ has the Ramsey property. We have to prove that $\operatorname{Forb}(G, H)$ does not have the Ramsey property in all the other cases. Assume that $G=$ $G_{0} \oplus G_{1} \oplus \cdots \oplus G_{m-1}=\bigoplus_{i \in m} G_{i}$ with $m \geq 2$ and for each $i \in m, \bar{G}_{i}$ is connected. Also, $H=H_{0}+H_{1}+\cdots+H_{n}=\sum_{i \in n} H_{i}$ with $n \geq 2$ and for each $i \in n, H_{i}$ is connected. We may assume that all of the graphs in $\left\{G_{i}: i \in\right.$ $m\} \cup\left\{H_{j}: j \in n\right\}$ have pairwise no vertex in common. It follows from Lemma 2 that if there is a graph $J \in \operatorname{Forb}(G, H)$ such that for all $i \in m$ and $j \in n$ there is an embedding from $G_{i}$ and an embedding from $H_{j}$ into $J$, then $\operatorname{Forb}(G, H)$ does not have the Ramsey property. From now on we will assume that there is no graph $J \in \operatorname{Forb}(G, H)$ such that for all $i \in m$ and $j \in n$ there is an embedding from $G_{i}$ and an embedding from $H_{j}$ into $J$. Many of the subsequent arguments will be by contradiction. The contradiction will be obtained by constructing a graph $J \in \operatorname{Forb}(G, H)$ such that for all $i \in m$ and $j \in n$ there is an embedding from $G_{i}$ and an embedding from $H_{j}$ into $J$. Under the assumption that there is no such graph $J$ and the assumption that $\operatorname{Forb}(G, H)$ has the Ramsey property we will analyse the structure of the graphs $G$ and $H$ and eliminate more and more possibilities until only the four exceptions remain.

If $\mathscr{G}$ is a family of graphs, then the family $\mathscr{B} \subseteq \mathscr{G}$ of graphs is a base of $\mathscr{G}$ if and only if for every graph $R \in \mathscr{G}$ there is a graph $B \in \mathscr{B}$ such that $R$ can be embedded into $B$ and if $B$ and $C$ are two different graphs in $\mathscr{B}$ then $B$ cannot be embedded into $C$. For $I \subset m$ and $L \subset n$ with $0<|I|<m$ and $0<|L|<n$ let $D^{I}=\bigoplus_{i \in I} G_{i}, C^{I}=G-D^{I}, A^{L}=\sum_{j \in L} H_{j}$ and $B^{L}=H-A^{L}$. We will prove over several of the following paragraphs that there exist subsets $I \subset m$ and $L \subset n$ with $0<|I|<m$ and $0<|L|<n$ such that the four graphs $A^{L}, B^{L}, C^{I}, D^{I}$ have a base containing at most two graphs. We will prove this assertion by contradiction. That is, we will assume for a contradiction that there are no two subsets $I \subset m$ and $L \subset n$ with $0<|I|<m$ and $0<|L|<n$ such that the four graphs $A^{L}, B^{L}, C^{I}$, $D^{I}$ have a base containing at most two graphs.

Choose an $I_{0} \subset m$ and $L_{0} \subset n$ with $0<\left|I_{0}\right|<m$ and $0<\left|L_{0}\right|<n$. Let $A=A^{L_{0}}, B=B^{L_{0}}, C=C^{I_{0}}$ and $D=D^{I_{0}}$. Note that $H=A+B, G=$ $C \oplus D$ and that none of the graphs $A, B, C, D$ is the empty graph. The set $\{A, B, C, D\}$ of graphs cannot have a base containing only one graph because this graph could then be taken as a graph $J$ into which all the component graphs $G_{i}$ and $H_{j}$ can be embedded. If there is an embedding $\alpha$ from $H$ into $D+C$ then there is a subset $L_{1} \in n$ with $0<\left|L_{1}\right|<n$ such that the four graphs $\sum_{j \in L_{1}} H_{j}, H-\sum_{j \in L_{1}} H_{j}, C, D$ have the base $C, D$. To see this, let $L_{1}=\left\{j \in n: V\left(\alpha\left(H_{j}\right)\right) \subseteq V(D)\right\} . \quad L_{1} \neq n$ and $L_{1} \neq \varnothing$ because otherwise $H$ could be embedded into $D$ or $C$ and hence into $G$. Hence, 
because we assume that the set $\{A, B, C, D\}$ does not have a base consisting of two elements, there is no embedding from $H$ into $D+C$. "There is no embedding from $G$ into $A \oplus B$ " is the dual statement.

In this paragraph and the next we will show that there is no embedding from one of the graphs in $\{A, B, C, D\}$ into one of the other three graphs in $\{A, B, C, D\}$. For a contradiction, assume that there is an embedding from one of the graphs in $\{A, B, C, D\}$ into one of the other three graphs in $\{A, B, C, D\}$. Say, there is an embedding $\beta$ from $C$ into one of the graphs in $A, B, D$. It suffices to investigate this case as the other cases arise either by renaming the graphs in $A, B, C, D$ or by duality. Let the graph $J$ be given by $J=D+(A \oplus B)$. Clearly all the component graphs $G_{i}$ and $H_{j}$ can be embedded into $J$. We will prove that $J \in \operatorname{Forb}(G, H)$ which is the desired contradiction. Let us assume to the contrary that there is an embedding $\alpha$ from $G$ into $J$. If $\alpha$ maps a vertex $c$ of $C$ into $D$, then $\alpha$ embeds all of $D$ into $D$ because $c$ is in $G$ adjacent to every vertex of $D$ and no vertex of $A \oplus B$ is adjacent to any vertex of $D$. But $\alpha$ would then embed one more vertex than $D$ contains into $D$, which is impossible because $\alpha$ is injective. If $\alpha$ embeds a vertex of $D$ into $D$, then $\alpha$ embeds all of $C$ into $D$, which according to the previous argument is impossible. This implies that $\alpha$ embeds $G$ into $A \oplus B$ which we have already ruled out. Hence $J \in \operatorname{Forb}(G)$.

We prove next that $J \in \operatorname{Forb}(H)$. Assume to the contrary that there is an embedding $\alpha$ from $H$ into $J$. If $\alpha$ maps a vertex $a_{1}$ of $A$ into $A$ and some other vertex $a_{2}$ of $A$ into $B$, then $\alpha$ would have to embed $B$ into $D$ which would make $A, D$ into a base of $\{A, B, C, D\}$. We assume therefore that $\alpha$ does not map a vertex $a_{1}$ of $A$ into $A$ and some other vertex $a_{2}$ of $A$ into $B$. Likewise we can assume that $\alpha$ does not map a vertex $b_{1}$ of $B$ into $A$ and some other vertex $b_{2}$ of $B$ into $B$. If $\alpha$ maps a vertex of $A$ into $A$ it cannot map a vertex of $B$ into $B$ and if $\alpha$ maps a vertex of $A$ into $B$ it cannot map a vertex of $B$ into $A$. Hence $\alpha$ embeds $A+B=H$ into $A+D$ or $\alpha$ embeds $A+B$ into $B+D$. We may assume without loss of generality that $\alpha$ embeds $A+B$ into $A+D$. It follows then from Lemma 4 that there is an embedding from $B$ into $D$. Again $\{A, D\}$ would then be a base of $\{A, B, C, D\}$. Hence $J \in \operatorname{Forb}(G, H)$ and we can conclude that there is no embedding from one of the graphs in $\{A, B, C, D\}$ into one of the other three graphs in $\{A, B, C, D\}$. This leaves us with the case that there is no embedding from one of the graphs in $\{A, B, C, D\}$ into some other one of the graphs in $A, B, C, D$. Also, there is no embedding from $G$ into $A \oplus B$ and there is no embedding from $H$ into $A+B$. Consider the graph $J$ with vertex set $V(J)=V(G) \cup V(H)$. The edges of $J$ are such that $J|(V(A) \cup V(B))=A \oplus B, J|(V(C) \cup V(D))=C+D, J \mid V(A) \cup V(C))=A+C$, $J|(V(A) \cup V(D))=A \oplus D, J|(V(B) \cup V(D))=B+D, J \mid(V(B) \cup V(C))=B \oplus C$. Clearly all the component graphs $G_{i}$ and $H_{j}$ can be embedded into $J$. We will prove that $J \in \operatorname{Forb}(G, H)$ which is the final contradiction and eliminates this case as well. To this end it suffices to prove that $J \in \operatorname{Forb}(H)$. The proof that $J \in \operatorname{Forb}(G)$ is the dual argument.

We assume to the contrary that $\alpha$ is an embedding from $H$ into $J$. There cannot be two vertices $x$ and $y$ of $A$ such that $\alpha(x)$ and $\alpha(y)$ are in different graphs of $A, B, C, D$. To see this we consider the six pairs of graphs in $A$, 
$B, C, D$. If $\alpha(x) \in V(A)$ and $\alpha(y) \in V(B)$ then every vertex of $J$ is adjacent to either $\alpha(x)$ or $\alpha(y)$. But no vertex of $\alpha(B)$ can be adjacent to any vertex of $\alpha(A)$. If $\alpha(x) \in V(A)$ and $\alpha(y) \in V(C)$ then $\alpha$ embeds $B$ into $A+C$. The graph $B$ cannot be embedded into $A$ or $C$ and hence $\alpha$ maps at least one vertex of $B$ into $C$ and at least one vertex of $B$ into $A$. This in turn implies that $\alpha$ maps $A$ into $A+C$. Using Lemma 4, it follows that $\alpha$ embeds $B$ into $C$. If $\alpha(x) \in V(A)$ and $\alpha(y) \in V(D)$ then $\alpha$ embeds $B$ into $C$. The situation in which the vertex $\alpha(x) \in V(B)$ and $\alpha(y) \in V(A)$ is of course the same as $\alpha(x) \in V(A)$ and $\alpha(y) \in V(B)$. If $\alpha(x) \in V(B)$ and $\alpha(y) \in V(C)$ then $\alpha$ embeds $B$ into $D$. If $\alpha(x) \in V(B)$ and $\alpha(y) \in V(D)$ then $\alpha$ embeds $B$ into $B+D$. Again $\alpha$ must map at least one vertex of $B$ into $B$ and at least one vertex of $B$ into $D$. Hence $\alpha$ embeds $A$ into $B+D$. Then the map $\alpha$ embeds $A+B=H$ into $B+D$ and hence by Lemma 4 embeds $A$ into $D$. If $\alpha(x) \in V(C)$ and $\alpha(y) \in V(D)$ then $\alpha$ maps $B$ into $C+D$. Hence it maps one vertex of $B$ into $C$ and one vertex of $B$ into $D$. Then $\alpha$ maps $H=A+B$ into $C+D$. We conclude that $\alpha$ embeds $A$ into one of the graphs of $\{A, B, C, D\}$. Likewise we get that $\alpha$ embeds $B$ into one of the graphs of $\{A, B, C, D\}$. Because of our assumption such that there is no embedding from one of the graphs in $\{A, B, C, D\}$ into some other one of the graphs in $\{A, B, C, D\}, \alpha$ would embed $A$ into $A$ and $B$ into $B$. This is impossible because in $H$ no vertex of $A$ is adjacent to any vertex of $B$ while in $J$ every vertex of $A$ is adjacent to every vertex of $B$.

We have proven that the set $\{A, B, C, D\}$ has a base consisting of two graphs. Up to renaming there are only three possibilities. The base is $\{A, B\}$ or $\{C, D\}$ or $\{A, D\}$. If there is a graph $J \in \operatorname{Forb}(G, H)$ such that both graphs in the base can be embedded into $J$, then for all $i \in m$ and $j \in n$ there is an embedding from $G_{i}$ and an embedding from $H_{j}$ into $J$. Hence we can assume that there is no graph $J \in \operatorname{Forb}(G, H)$ such that both graphs in the base can be embedded into $J$. This implies in particular that none of the two graphs of the base can be embedded into the other.

Case 1: $\{A, B\}$ is the base of $\{A, B, C, D\}$. Let us first consider the possibility that $G=C \oplus D \cong K_{1} \oplus K_{1}=K_{2}$. Then $H$ and every other graph in $\operatorname{Forb}(G)$ consists of a set of pairwise not adjacent vertices. A graph in $\operatorname{Forb}(G, H)$ contains then at most $|V(H)|-1 \geq 1$ vertices. Hence if $|V(H)|=$ 2, $\operatorname{Forb}(G, H)$ is the trivial family consisting only of the singleton graph and if $|V(H)|>2, \operatorname{Forb}(G, H)$ is not Ramsey. The graph $H$ contains at least two vertices because otherwise either $A$ or $B$ would be the empty graph. We assume from now on that $|V(G)| \geq 3$.

We will prove that one of the two graphs $C$ or $D$, say $C$, consists of a single vertex $u$ and that the other graph, $D$, contains an isolated vertex $v$. Observe that in this case the vertex $u$ would be adjacent to every other vertex of $G$ and that the vertex $v$ would be only adjacent to the vertex $u$. Let $a \in V(A)$ and $b \in V(B)$ be two vertices. Consider the graph $J$ with vertex set $V(J)=V(A) \cup V(B)$. The edges of $J$ are such that $J \mid V(A)=A$ and $J \mid V(B)=B$. The vertex $x \in V(A)$ is adjacent to the vertex $y \in V(B)$ if and only if $x=a$ and $y=b$. Clearly, the graphs $A$ and $B$, and hence also the graphs $C$ and $D$, can be embedded into the graph $J$. The graph $J \in \operatorname{Forb}(H)$, because $H$ and $J$ have the same number of vertices but cannot be isomorphic because $J$ has one more edge than $H$. We will prove that $G$ 
cannot be embedded into $J$ unless it contains a vertex, say $u$, which is adjacent to every other vertex of $G$ and a vertex, say $v$, which is only adjacent to the vertex $u$. Let $\alpha$ be an embedding from $G$ into $J$. Because $|V(G)| \geq 3$ there is some vertex of $G$ which does not get mapped by $\alpha$ to $a$ or $b$. Assume without loss of generality that there is a vertex $d \in V(D)$ such that $a \neq \alpha(d) \in V(A)$. The embedding $\alpha$ then embeds $C$ into $A$. If for some vertex $c \in V(C)$, $\alpha(c) \neq a$, then $\alpha$ would embed $D$ and hence $G=C \oplus D$ into $A$. But then $G$ would have an embedding into $H$. Hence $C$ consists of a single vertex $u$ and $\alpha(u)=a$. If $G, u$ is adjacent to every other vertex of $G$. The only vertex of $B$ which is adjacent to $a=\alpha(u)$ is the vertex $b$. The graph $G$ cannot be embedded into $A$ and hence $\alpha(G)$ contains at least one vertex of $B$. Hence the vertex $v=\alpha^{-1}(b)$ is in $G$ only adjacent to the vertex $u$. It follows that $G \in \mathfrak{M}$.

We have argued that the conditions of Lemma 7 are satisfied and hence Lemma 7 eliminates all graphs $G$ except for $G \cong P_{2}$. If $R$ is a graph in Forb $\left(P_{2}\right)$ then the adjacency relation in $R$ is transitive. Hence $R$ is the sum $(+)$ of complete graphs. In particular $H$ is the sum of complete graphs. It is not possible that $H \cong K_{1}+K_{1}$ because otherwise $H$ could be embedded into $G \cong P_{2}$. If $H$ contains $s \geq 3$ connected components let $r \in \omega$ be larger than the number of vertices in any of the connected components of $H$. Let $R$ be the sum of $s-1$ complete graphs with $r$ vertices each and note that $R \in \operatorname{Forb}(G, H)$. It is not difficult to see that there is no graph $L \in \operatorname{Forb}(G, H)$ such that $L \rightarrow(R)_{2}^{1}$. (Color every vertex of the largest component of $L$ red and every other vertex blue. There is no embedding of $R$ into the red vertices because $R$ contains more than one component. If there is an embedding from $R$ into the blue vertices then there is an embedding from $H$ into $L$.) The number $s$ is at least 2 because $H$ is the sum of two nonempty graphs. If $s=2$, let $t \geq p$ be the number of vertices of the two-connected components of $H$. There is no graph $L \in \operatorname{Forb}(G, H)$ such that $L \rightarrow\left(K_{t-1}+K_{t-1}\right)_{2}^{1}$. (Color exactly one vertex of every component red.)

This completes the discussion of Case 1 . We note that in Case 1 the only family $\operatorname{Forb}(G, H)$ which has the Ramsey property is the trivial family $\mathscr{E}_{4}$ consisting only of the singleton graph. This family is clearly self-dual.

Case 2: $\{C, D\}$ is the base of $\{A, B, C, D\}$. This case is dual to Case 1 . Hence, in Case 2 , the only family $\operatorname{Forb}(G, H)$ which has the Ramsey property is the self-dual family $\mathscr{E}_{4}$.

Case 3: $\{A, D\}$ is the base of $\{A, B, C, D\}$. Recall that we assume that there is no graph $J \in \operatorname{Forb}(G, H)$ such that the four graphs $\{A, B, C, D\}$ can be embedded into $J$ and hence that there is not graph $J \in \operatorname{Forb}(G, H)$ such that the two graphs $\{A, D\}$ can be embedded into $J$. Also, we wish to eliminate all those pairs $G, H$ of graphs for which $\operatorname{Forb}(G, H)$ does not have the Ramsey property. Here $H=A+B$ and $G=D \oplus C$. From Lemma 8 we get then that the graphs $B$ and $C$ are both the singleton graph and (i) or (ii) holds:

(i) $D$ is of the form $D=M+K_{1}$ are there is an embedding from $M$ into $A$.

(ii) $A$ is of the form $A=M \oplus K_{1}$ and there is an embedding from $M$ into $D$. 
The statement (i) is dual to statement (ii) and hence it is sufficient to determine all the Ramsey families $\operatorname{Forb}(G, H)$ satisfying (i) and then to take their complements to determine all the Ramsey families $\operatorname{Forb}(G, H)$ satisfying (ii). We will assume that $D$ is of the form $D=M+K_{1}$ and there is an embedding from $M$ into $A$. We will denote the isolated vertex of $D$ by $v$, the vertex of $G$ which is adjacent to all the other vertices of $G$ by $u$ and the single vertex in $B$ by $w$. Observe that $V(C)=\{u\}$ and that $V(B)=\{w\}$. Because $M$ can be embedded into $A$ we get that $|V(A)| \geq|V(M)|$. If $|V(A)|=|V(M)|$ then $A \cong M$ and hence $M$ and therefore $D$ embeds $A$ contrary to our assumptions. Hence $|V(A)|>|V(M)|$. Lemma 18 tells us that if $|V(M)|=1$, then $\operatorname{Forb}(G, H)$ has the Ramsey property if and only if $\operatorname{Forb}(G, H)=\mathscr{E}_{1}$. We assume therefore from now on that $|V(M)|>1$.

Assume that $A \cong P_{2}$ and $M$ consists of a set of pairwise not adjacent vertices. The graph $M$ can be embedded into $A$ and hence $M \cong \bar{K}_{2}$. Then $G \cong K_{1} \oplus\left(K_{1}+K_{1}+K_{1}\right)$ and $H \cong K_{1}+P_{2}$ and hence $\operatorname{Forb}(G, H)=\mathscr{E}_{3}$. We can therefore assume that if $A \cong P_{2}$ then $M$ does not consist of a set of pairwise not adjacent vertices. Then according to Lemma 10 there is a connected graph $R \in \operatorname{Forb}(G)$ into which $D$ can be embedded but $A$ cannot be embedded and hence we deduce from Lemma 11 that $A$ is an $M$-tree. Note that therefore, if $M$ is a complete graph then $A$ is a complete graph.

We investigate first the case $|V(M)|=2$. If $V(M)=\{p, x\}$ and the vertices $p$ and $x$ are not adjacent then, because $M$ can be embedded into $A, A$ contains two nonadjacent vertices $a$ and $y$. The function $\delta=\{(x, y)\}$ is an embedding from $M-p$ into $A$ such that no vertex of $\delta(M-p)$ is adjacent to the vertex $a$. Because $M$ is not complete, we deduce from Lemma 12 that in this case $\operatorname{Forb}(G, H)=\mathscr{E}_{3}$. If $M$ is the complete graph on two vertices we get from Lemma 15 that $|V(A)|<4$. If $|V(A)|=3$ then $\operatorname{Forb}(G, H)=\mathscr{E}_{2}$. If $|V(A)|=2$ then the graph $Y$ can be embedded into the graph $G$. If $|V(A)|=1$ then $M$ could not be embedded into $A$.

We can now assume that $|V(M)|>2$ and hence that $|V(A)|>|V(M)|>2$. We deduce from Lemma 13 that in the only remaining cases $M$ is a complete graph. Hence $A$ is a complete graph as well. Using Lemma 14 and Lemma 15 we get $|V(A)|=2 m-1$. Lemma 16 then restricts us to the case $|V(M)|=3$ and $|V(A)|=5$, which is then dealt with in Lemma 17 .

\subsection{Proof of Lemma 7 to settle Case 1}

Lemma 7. Let $G \in \mathfrak{M}-P_{2}$ with cutpoint $u$ adjacent to all other vertices of $G$ be such that $G-u$ contains a connected component which consists of the single element $v$. The graphs $A, B \in \operatorname{Forb}(G)$ are such that there is no graph $J \in \operatorname{Forb}(G, A+B)$ which embeds both $A$ and $B$. Then $\operatorname{Forb}(G, A+B)$ is not Ramsey.

Proof. Assume to the contrary that $\operatorname{Forb}(G, A+B)$ is Ramsey. Let $M=G-$ $u-v, D=G-u, n=V(G)$ and $\mathscr{N}=\mathscr{N}_{\mathscr{G}}$ the set of chromatically bounded graphs in $\operatorname{Forb}(G)$. We will show in the paragraph after the next that $N \in \mathcal{N}$ if and only if $N$ embeds $A+B$ and hence that under the assumptions of the present lemma $\mathscr{N} \neq \varnothing$. (In any case according to Theorem 5.1 of [4] (see Theorem 6 of this paper), $\operatorname{Forb}(G)$ is not Ramsey and hence $\mathscr{N} \neq \varnothing$.)

First we deal with a few special cases, namely the cases that $n \leq 3$. Clearly 
$n \leq 1$ is not possible because $G$ contains the two different vertices $u$ and $v$. If $n=2, G \cong K_{2}$ then $A+B$ is a graph without edges which in turn implies that $\operatorname{Forb}(G, A+B)$ is a finite set of graphs and hence does not have the Ramsey property. If $n=3$ then $G \cong P_{2}$ in contradiction to $G \in \mathfrak{M}-P_{2}$.

We will prove that $A+B$ is the unique graph in $\mathscr{N}$ with the smallest number of vertices. This will follow if we can show that $N \in \mathcal{N}$ if and only if there is an embedding from $A+B$ into $N$. So, assume that there is an embedding from $A+B$ into $N \in \operatorname{Forb}(G)$. If $N \notin \mathscr{N}$, then by Theorem $5, N$ can be disjointly amalgamated with $N$ on any pair of vertices of $N$ into a graph $J \in \operatorname{Forb}(G)$. Hence $A$ can be amalgamated with $B$ on any pair of vertices into a graph $J \in \operatorname{Forb}(G)$ with $|V(J)| \leq|V(A)|+|V(B)|-1$. This would mean that $J \in \operatorname{Forb}(G, A+B)$ contrary to the assumption that there is no graph $J \in \operatorname{Forb}(G, A+B)$ into which both graphs $A$ and $B$ can be embedded. If on the other hand for some $N \in \mathcal{N}$ there is no embedding from $A+B$ into $N$ then $N \in \operatorname{Forb}(G, A+B)$. Because we assumed that $\operatorname{Forb}(G, A+B)$ has the Ramsey property there is for every $r \in \omega$ a graph $J \in \operatorname{Forb}(G, A+B) \subseteq \operatorname{Forb}(G)$ with $J \rightarrow(N)_{r}^{1}$, in contradiction to the definition of $\mathscr{N}$.

From here on we will proceed as follows. To every connected graph $R \in$ $\operatorname{Forb}(G)$, which embeds the graph $D=G-u$, we will construct a graph $L=R_{L}$ such that the graphs $R$ and $L$ cannot be disjointly amalgamated to a graph $J \in \operatorname{Forb}(G)$ on a given pair of vertices. Using the corollary to Theorem 5 we will conclude that $L+R \in \mathscr{N}$. Hence $A+B$ can be embedded into $L+R$. We will show that $A$ can be embedded into one of the two graphs $L$ and $R$, and $B$ into the other one. Say $A$ can be embedded into $L$ and $B$ into $R$. Using different graphs $R$ and the uniqueness of $A+B$ as the smallest graph in $\mathcal{N}$ we will determine the graph $B$. Then changing $R$ again we will determine that a different graph is equal to $B$ and hence arrive at a contradiction.

We construct for $r \in \omega$ the graph $L^{r} \in \operatorname{Forb}(G)$ as follows. Let $H$ be a $|V(M)|$-uniform hypergraph of girth $g$ larger than or equal to five and chromatic number $\chi(H)>r . L_{1}^{r}(H)$ is a graph constructed from the hypergraph $H$ by embedding $M$ into the edges of $H$. (For definitions see the paragraph before Lemma 5). Let $z_{0}$ and $v$ be two different vertices with $z_{0}, v \notin V(H) \cup V(M)$. Then $L^{r}=z_{0} \oplus L_{1}^{r}(H)$. Let $T$ be a dual component of $M$ with a maximal number of vertices. By Lemma 5 there is no embedding from $T+v$ into $L^{r}$. Hence $L^{r} \in \operatorname{Forb}(G-u)$ and hence $L^{r} \in \operatorname{Forb}(G)$. We will say that $H$ is the underlying hypergraph of $L^{r}$.

Let $Q \in \operatorname{Forb}(G)$ be a connected graph such that there is an embedding $\alpha$ from $G-u=D$ into $Q$ and $z$ a vertex in $V(Q)$. Let $r>\Xi_{z}(Q)$ be any number and put $Q_{L}=L^{r}=L$. It follows from Lemma 6 that if $J$ is any graph into which $L$ and $Q$ can be disjointly amalgamated on $z_{0} \simeq z$, then $J \notin \operatorname{Forb}(G)$. Later on we will refer to this fact as $\operatorname{stm}(J ; L, Q, z)$.

Next we will construct the graph $R$. If $G$ is not a star, or equivalently, if $\bar{M}$ is not a complete graph, choose a set $S \in V(M)$ in such a way that every connected component of $M$ has exactly one of its vertices in $S$. Let $z$ and $x$ be two vertices not in $G$. The graph $R \in \operatorname{Forb}(G)$ has vertex set $V(M) \cup\{x, z\}$. The edges of $R$ are such that $R \mid V(M)=M$. The vertex $z$ is in $R$ adjacent to the vertex $y \in V(R)$ if and only if $y \in S \cup\{x\}$. The vertex $x$ is only adjacent to the vertex $z$. Observe that the degree of a vertex of $R$ is 
at most $n-2$. The vertex $u$ has in $G$ degree $n-1$ and hence $R=\operatorname{Forb}(G)$. The graph $G-u=D$ can be embedded into $R$ because $R \mid(V(M) \cup\{x\})$ is isomorphic to $D$.

If $\bar{M}$ is complete, that is if $G$ is a star, let $x, y, z$ be three vertices not in $G$ and $w$ a vertex in $M$. We define $R \in \operatorname{Forb}(G)$ to be the graph with vertex set $V(M) \cup\{x, y, z\}$. The edges of $R$ are such that $R \mid V(M)=M$. The vertex $z$ is in $R$ adjacent to every vertex of $M$. The vertex $x$ is adjacent to $w$ and to $y$. The vertex $y$ is only adjacent to $x$. Observe again that the degree of a vertex of $R$ is at most $n-2$ because $2 \geq n-1$. The vertex $u$ has in the graph $G$ degree $n-1$ and hence $R \in \operatorname{Forb}(G)$. The graph $G-u=D$ can be embedded into $R$ because $R \mid(V(M) \cup\{y\})$ is isomorphic to $G-u$.

Let $L=R_{L}$ and note that $L+R=\mathscr{N}$. Otherwise we get from the corollary to Theorem 5 that $L+R$ could be disjointly amalgamated with $L+R$ on any pair of vertices to a graph $J \in \operatorname{Forb}(G)$. But then $L$ could be disjointly amalgamated with $R$ on $z_{0} \simeq z$ to a graph $J \in \operatorname{Forb}(G)$ contrary to $\operatorname{stm}(J ; L, R, z)$. Therefore, $A+B$ can be embedded into $L+R$. We will prove that $A$ can be embedded into one of the two graphs $L$ and $R$, and $B$ into the other one.

Assume that $\left\{A_{i}: i \in s\right\}$ is the set of connected components of $A$ and $\left\{B_{j}: j \in t\right\}$ is the set of connected components of $B$.

Claim 1. For $i \in s$ and $j \in t$ there is no graph $T \in \operatorname{Forb}\left(G, A_{i}+B_{j}\right)$ such that $T$ embeds $A_{i}$ and $B_{j}$.

Proof. If for some $i \in S$ and $j \in t$ there were a graph $T \in \operatorname{Forb}\left(G, A_{i}+B_{j}\right)$ such that both $A_{i}$ and $B_{j}$ can be embedded into $T$, we could assume, by restricting $T$ to the appropriate subgraph, that $V(T)=V\left(A_{i}\right) \cup V\left(B_{j}\right)$ and that $|V(T)| \leq\left|V\left(A_{i}\right)\right|+\left|V\left(B_{j}\right)\right|$. But then both the graphs $A$ and $B$ could be embedded into the graph $J \in \operatorname{Forb}(G, A+B)$ with $J=T+\left(A-A_{i}\right)+\left(B-B_{j}\right)$. The graph $G$ cannot be embedded into the graph $J$ because $G$ is connected and cannot be embedded into any of the connected components of $J$. If the graph $A+B$ could be embedded into $J$, then because $|V(A+B)| \geq|V(J)|$, $|V(A+B)| \cong|V(J)|$. But then $T \cong A_{i}+B_{j}$ contrary to $T \in \operatorname{Forb}\left(G, A_{i}+B_{j}\right)$. Hence there is no graph $T \in \operatorname{Forb}\left(G, A_{i}+B_{j}\right)$ such that both $A_{i}$ and $B_{j}$ can be embedded into $T$.

Claim 2. For $i \in s$ and $j \in t$ the graph $A_{i}$ does not embed the graph $B_{j}$ and $B_{j}$ does not embed $A_{i}$.

Proof. If, let us say, $A_{i}$ can be embedded into $B_{j}$ then $J=B_{j} \epsilon$ Forb $\left(G, A_{i}+B_{j}\right)$ and both graphs $A_{i}$ and $B_{j}$ can be embedded into $J$.

Claim 3. For $i \in s$ and $j \in t,\left|V\left(A_{i}+B_{u}\right)\right|>|V(G)|$.

Proof. If $\left|V\left(A_{i}+B_{j}\right)\right|<|V(G)|$ then $A_{i} \oplus B_{j} \in \operatorname{Forb}\left(G, A_{i}+B_{j}\right)$. If $\left|V\left(A_{i}+B_{j}\right)\right|=|V(G)|$ and because $A_{i} \oplus B_{j} \notin \operatorname{Forb}\left(G, A_{i}+B_{j}\right)$ then as we have shown above $G \cong A_{i} \oplus B_{j}$. Observe that neither $A_{i}$ nor $B_{j}$ are singleton graphs, otherwise they could be embedded into the other contrary to Claim 2 . Hence the graph $T$ which is equal to $A_{i} \oplus B_{j}$ minus an edge from $A_{i}$ to $B_{j}$ is a graph in $\operatorname{Forb}\left(A_{i}+B_{j}\right)$ and we conclude as before that $G$ is also isomorphic to $T$, a contradiction which establishes Claim 3 .

For $i \in s$ and $j \in t$ we will show that $L \in \operatorname{Forb}\left(G, A_{i}+B_{j}\right)$. Let us assume to the contrary that $\alpha$ is an embedding from $A_{i}+B_{j}$ into $L$. Because $z_{0}$ is adjacent to every other vertex of $L$, it cannot be in the image of $\alpha$. The graph $A_{i}$ is not the singleton graph, otherwise it could be embedded into $B_{j}$ 
contrary to Claim 2. The graph $B_{j}$ is not the singleton graph, otherwise it could be embedded into $A_{i}$. Assume that $a, b \in V\left(A_{i}\right)$ and $c, d \in V\left(B_{j}\right)$ are four vertices. Then each of the four pairs $\{\alpha(a), \alpha(c)\},\{\alpha(a), \alpha(d)\}$, $\{\alpha(b), \alpha(c)\}$ and $\{\alpha(b), \alpha(d)\}$ is contained in some hyperedge of $H$. The girth of $H$ is at least five and hence all four vertices $\alpha(a), \alpha(b), \alpha(c), \alpha(d)$ are contained in some hyperedge $E$ of $H$. Hence $V\left(\alpha\left(A_{i}+B_{j}\right)\right) \subseteq E$ and therefore $\left|V\left(A_{i}+B_{j}\right)\right|<|V(G)|$ contrary to Claim 3 .

Next we prove that also $R \in \operatorname{Forb}\left(G, A_{i}+B_{j}\right)$. Assume to the contrary that $\alpha$ is an embedding from $A_{i}+B_{j}$ into $R$. The graph $R$ is connected and the graph $A_{i}+B_{j}$ is disconnected. Hence, $\left|V\left(A_{i}+B_{j}\right)\right| \leq|V(R)|-1 \leq n=|V(G)|$, by the construction of the graph $R$.

For all $i \in s$ and $j \in t$ we have established that both graphs $L$ and $R$ are elements of $\operatorname{Forb}\left(G, A_{i}+B_{j}\right)$ and that $A+B$ can be embedded into $L+R$. This implies that $A$ can be embedded into one of the two graphs $L$ and $R$, and $B$ into the other one. To be specific let us assume that $A$ can be embedded into $L$ and $B$ into $R$. Note that no connected component of $B$ can be embedded into $L$.

The previous argument shows that $A$ can be embedded into $L^{r}$ for every number $r$ with $r>\Xi_{z}(R)$ and no connected component of $B$ can be embedded into $L^{r}$. This in turn means that if $Q \in \operatorname{Forb}(G)$ is any connected graph such that $G-u=D$ can be embedded into $Q$, then $B$ can be embedded into $Q$. In order to see this choose a vertex $z \in V(Q)$ and a graph $L=L^{r}$ such that $r>\max \left(\Xi_{z}(Q), \Xi_{z}(R)\right)$. Then $L+Q \in \mathscr{N}$ and hence $A+B$ can be embedded into $L+Q$. The graph $A$ can be embedded into $L$ and no connected component of $B$ together with $A$ can be embedded into $L$. Hence $B$ can be embedded into $Q$.

Claim 4. The graph $D$ can be embedded into the graph $B$ and hence $n \leq$ $|V(B)|$.

Proof. Observe that if $r \in \omega$ and that if $P$ is any graph such that $D$ cannot be embedded into $P$, then $L^{r} \oplus P \in \operatorname{Forb}(D)$ and hence $L^{r} \oplus P \in$ Forb $(G)$. We will use this idea to show that $D$ can be embedded into $B$. If not, $L^{r} \oplus B \in \operatorname{Forb}(G)$ and because $L^{r} \oplus B \in \operatorname{Forb}(A+B)$ it follows that $L^{r} \oplus B \in \operatorname{Forb}(G, A+B)$. Then both $A$ and $B$ can be embedded into $J=$ $L^{r} \oplus B \in \operatorname{Forb}(G, A+B)$ because $A$ can be embedded into $L^{r}$. But this is contrary to the assumptions. If $D \cong B$, then every connected component of $M$ is a connected component of $B$. The subgraph $M$ of $D$ can be embedded into $L$ and hence a connected component of $B$ could be embedded into $L$. We conclude that $n \leq|V(B)|$.

Case 1. The graph $G$ is not a star.

If $G$ is not a star, then $|V(R)|=n$. The graph $R$ embeds $B$ and hence $R$ and $B$ are isomorphic. Assume first that $M$ contains at least two edges. The graph $G$ contains in this case at least two more edges than $R$. Let $Q$ be a graph obtained from $R$ by adding in $R$ one more edge from $z$ to $M$. The graph $Q$ is not isomorphic to $R$ and does not embed $G$ but $Q$ embeds $D$. Hence $Q$ embeds $B$ and because $B$ and $Q$ have the same number of vertices, $B$ and $Q$ are isomorphic. But this is impossible because $R$ and $Q$ are not isomorphic.

We assume next that $M$ contains exactly one edge $\{a, b\}$. One of $a$ and $b$, say $b$, is an element of $S$. That is $M$ consists of $n-4$ isolated vertices and the 
edge from $a$ to $b$, and $D$ consists of $n-3$ isolated vertices and the edge from $a$ to $b$. Let $\{x, y, z\}$ be a set of three vertices with $V(M) \cap\{x, y, z\}=$ $\varnothing$ and $Q$ the graph with $V(Q)=V(M) \cup\{x, y, z\}$ and edgeset such that $V(Q) \mid M=M$, the vertex $z$ is adjacent to all vertices in $V(M)-\{a\}$, the vertex $x$ is adjacent to the vertices $a$ and $y$, and $y$ is only adjacent to the vertex $x$. There are no other adjacencies. The graph $G$ embeds a triangle and $Q$ does not embed a triangle. Hence $Q \in \operatorname{Forb}(G)$. On the other hand $Q$ embeds $D$. We deduce that $Q$ embeds $B$. The graph $B$ is isomorphic to $R$ and hence contains a vertex of degree $n-2$. The graph $Q$ does not contain a vertex of degree $n-2$, unless $n-2=2$. If $n=4, G=u \oplus(M+v)$ and $M$ is the edge $\{a, b\}$, and because $B$ is isomorphic to $R, B \cong P_{3}$. Observe that because $M$ is complete, $L$ is complete and hence $A$ is a complete graph. Put $|V(A)|=m$. If $m \leq 2, A$ and of course $B$ can be embedded into $B \in \operatorname{Forb}(G, A+B)$. Assume therefore that $m \geq 3$.

In the case where the graph $G$ is not a star we have reduced the possibilities for the graphs $G, A$ and $B$ to the case where $G \cong\left(K_{2}+v\right) \oplus u, B \cong P_{3}$, $M \cong K_{2}$ and $A$ is a complete graph on $m \geq 3$ vertices. We assume that we are in exactly this situation and want to prove that $\operatorname{Forb}(G, A+B)$ does not have the Ramsey property. First we will show that if a vertex $a$ of a graph $W \in \operatorname{Forb}(G)$ is in a connected component of $W$ which contains a triangle $\Gamma$, then $a$ is adjacent to at least one vertex of $\Gamma$. We proceed by considering the shortest path from the triangle to the vertex $a$. If the path has length one, $a$ is adjacent to a vertex of $\Gamma$. If $c, b, a$ is the shortest path from $\Gamma$ to $a$ with $c \in V(\Gamma)$, there is a vertex $d \in V(\Gamma)$ with $d \neq c$ which is adjacent to $b$. Otherwise $G$ can be embedded into $W$. But then $\{c, d, b, a\}$ span a copy of $G$ unless $c$ or $d$ is adjacent to $a$. Hence there is no vertex in $W$ which has distance two from $\Gamma$ which proves that $a$ is adjacent to at least one vertex of $\Gamma$.

Also, if an edge $\{a, b\}$ of $W$ is in a connected component of $W$ which contains a triangle $\Gamma$, then the edge $\{a, b\}$ is contained in some triangle of $W$. If $a$ is a vertex of the triangle $\Gamma$, there is a vertex $d \in V(\Gamma)$ which is adjacent to $b$, otherwise $G$ can be embedded into $W$. Then $\{a, b, d\}$ form a triangle. If neither $a$ nor $b$ are in $\Gamma$, then there is an edge from $a$ to some vertex $d$ of $\Gamma$ and we already proved that there is then some triangle $\Lambda$ containing the edge $\{a, d\}$. The edge $\{a, b\}$ is contained in some triangle because $a$ is a vertex of the triangle $\Lambda$.

Finally we need to know that if a connected component $C$ of $W$ contains a triangle then the graph $K_{1}+K_{2}$ cannot be embedded into $C$. Assume to the contrary that $C \mid(\{a, b, c\}) \cong K_{1}+K_{2}$ with $a$ adjacent to $b$. There is then a vertex $d$ adjacent to $a$ and $b$ to form the triangle $\{a, b, d\}$. The vertex $c$ is adjacent to at least one of the vertices of this triangle $\{a, b, d\}$ and because $c$ is not adjacent to $a$ or $b, c$ is adjacent to $d$ only. But the vertices $a, b$, $c, d$ span a copy of $G$.

The graph $K_{1}+K_{2}+K_{3}$ is a graph of the family $\operatorname{Forb}\left(G, K_{m}+P_{3}\right)$. For $D \in \operatorname{Forb}\left(G, K_{m}+P_{3}\right)$ colour all of the vertices of $D$ which are in a connected component of $D$ containing a triangle red and all other vertices blue. $K_{1}+$ $K_{2}+K_{3}$ cannot be embedded into one of the colour classes. Hence, the family $\operatorname{Forb}\left(G, K_{m}+P_{3}\right)$ of graphs is not Ramsey.

Case 2. The graph $G$ is a star. 
The remaining case is that the graph $G$ is a star. Remember that $n>3$. In this case the graph $R$ contains $n+1$ elements, $|V(B)| \geq n$ and $R$ embeds $B$. Hence $|V(B)|=n$ or $|V(B)|=n+1$ and $B$ is isomorphic to $R$. Assume first that $|V(B)|=n$. The graph $B$ is then isomorphic to $R-a$ for some vertex $a \in V(R)$. The vertex $a$ cannot be equal to $z$ because $R-z$ contains a singleton vertex which can be embedded into $L$. This is in contradiction to a previous observation that no connected component of $B$ can be embedded into $L$. If $a \in V(M)$ then $R-a$ does not embed $D$. If $a=x$, then $\{y\}$ is a connected component of $R-a \cong B$ which can be embedded into $L$. If $a=y$ then $R-a$ does not embed $D$, contrary to Claim 4. Hence we are left with the situation that $B$ is isomorphic to $R$.

If the graph $B$ is isomorphic to the graph $R$ consider the path $Q$ with $2 n-3$ vertices. It is connected and $D=G-u \cong \bar{K}_{n-1}$ can be embedded into it. Because $G$ is not isomorphic to $P_{2}$, because $G \in \mathfrak{M}-P_{2}$ by assumption, $G$ cannot be embedded into $Q$. If $n>4, B \cong R$ cannot be embedded into the path $Q$, because $R$ contains the vertex $z$ which has degree $n-2$. Hence we can assume that $G$ is a star with four vertices. If $G$ is a star with four vertices let $Q$ be the graph on the five vertices $\{a, b, c, d, e\}$ in which the vertices $a, b, c$ span a triangle and $d, e$ are vertices of degree one, $d$ being adjacent to $a$ and $e$ to $b$. The graph $Q$ does not contain $B \cong P_{4}$, the path of length four, nor the star on four vertices as spanning subgraphs, but it is connected and contains three pairwise nonadjacent vertices and therefore $D$. Hence $B \cong Q$ which is not possible because $P_{4} \cong Q$.

\subsection{A PRELIMINARY CHARACTERIZATION OF THE GRAPHS WHICH SATISFY CASE 2}

Lemma 8. Let $A, B, C, D$, none of them the empty graph, be four graphs in the family $\operatorname{Forb}(A+B, C \oplus D)$ of graphs. If there is no graph $J \in \operatorname{Forb}(A+B, C \oplus D)$ into which both graphs $A$ and $D$ can be embedded then the graphs $B$ and $C$ are both the singleton graph and (i) or (ii) holds:

(i) $D$ is of the form $D=M+K_{1}$ and there is an embedding from $M$ into $A$.

(ii) $A$ is of the form $A=M \oplus K_{1}$ and there is an embedding from $M$ into $D$.

Proof. Note that there cannot be an embedding from $A$ into $D$ and that there cannot be an embedding from $D$ into $A$.

Let $A_{1}$ be an induced subgraph of $A$ such that there is an embedding $\beta$ from $A_{2}=A-A_{1}$ into $D$ and a vertex $a \in V\left(A_{1}\right)$ such that for all vertices $x \in V\left(A_{1}-a\right)$ and $y \in V\left(A_{2}\right), x$ is not adjacent to $y$. Such a situation always exists. Just take $A$ for $A_{1}$ and any vertex in $A$ for $a$. Choose, under the above conditions, the pair $A_{1}, a$ so that first $\left|V\left(A_{1}\right)\right|$ is minimal and then so that the number of edges from $a$ to $A_{2}$ is maximal. Observe that if $a$ is adjacent to a vertex of $A_{2}$ then $a$ is a cutpoint of the connected component of $A$ containing $a$. If $a$ is not adjacent to any vertex of $A_{2}$, then $A_{1}$ is connected due to the minimality of $\left|V\left(A_{1}\right)\right|$. Let $J$ be the graph with vertex set $V(J)=$ $V\left(A_{1}\right) \cup V(D)$. The edges of $J$ are such that $J\left|V\left(A_{1}\right)=A_{1}, J\right| V(D)=D$, and such that the function $\sigma$ from $A$ into $J$, with $\sigma$ restricted to $A_{1}$ being the identity function and $\sigma$ restricted to $A_{2}$ the embedding $\beta$, is an embedding 
from $A$ into $J$. Also, for $x \in V\left(A_{1}-a\right)$ and $y \in V(D)-V\left(\beta\left(A_{2}\right)\right), x$ and $y$ are not adjacent but $a$ is adjacent to every vertex $y \in V(D)-V\left(\beta\left(A_{2}\right)\right)$.

We prove first that there is no embedding $\alpha$ from $A+B$ into $J$. For a contradiction, let $\alpha$ be such an embedding. The vertex $a$ is an element of $V(\alpha(A))$. Otherwise, if $a \notin V(\alpha(A))$ and because $\alpha$ does not embed $A$ into $D$ it would follow that $\alpha$ embeds a component $T$ of $A$ into $A_{1}-a$. For any vertex $a_{1}$ of $T, \alpha$ embeds $A-T$ into $D$ and for all vertices $x \in V\left(T-a_{1}\right)$ and $y \in V(A-T), x$ is not adjacent to $y$. This violates the minimality of $A_{1}$. This implies that for any vertex $b \in V(B), \alpha(b) \in V\left(A_{1}\right)-a$, or $\alpha(b) \in V(D)$ and $\alpha(b)$ is not adjacent to $a$. If $\alpha(b) \in V\left(A_{1}\right)-a, \alpha$ embeds $V(A)$ into $V\left(A_{1}-\alpha(b)\right) \cup V(D)$ in contradiction to the minimality of $A_{1}$. If $\alpha(b) \in V(D)$ and $\alpha(b)$ is not adjacent to $a$ then again because of the minimality of $A_{1}, V\left(A_{1}\right) \subseteq V(\alpha(A))$. Then the number of edges in $A$ between $a$ and $A_{2}$ is smaller than the number of edges in $\alpha(A)$ between $a$ and $\alpha(A)-A_{1}$ in contradiction to the assumption on the maximality of the number of edges between $a$ and $A_{2}$.

Next we will investigate the conditions under which $C \oplus D$ can be embedded into $J$. Assume that $\alpha$ is an embedding from $C \oplus D$ into $J$. Let $c \in V(C)$. If $\alpha(c) \in V\left(A_{1}-a\right)$, then because $c$ is in $C \oplus D$ adjacent to every vertex of $D, \alpha$ would embed $D$ into $A_{1}$. Hence no vertex of $C$ is mapped by $\alpha$ into $A_{1}-a$. If $\alpha(c) \in V(D)$ no vertex of $D$ can be mapped by $\alpha$ into $A_{1}-a$, which implies that $\alpha$ maps $V(C \oplus D)$ into $V(D) \cup\{a\}$. Using cardinality considerations we conclude that this is only possible if $|V(C)|=1$. The remaining possibility is $|V(C)|=1$ with $\alpha(c)=a$. In any case we can conclude that $|V(C)|=1$ holds.

By dualising the above considerations to the complements we get that also $|V(B)|=1$ holds. (The conditions of the lemma we are proving are self-dual. Hence for the dual argument we would break $D$ into $D_{1}$ and $D_{2}=D_{1}-D_{2}$ such that $\ldots)$. We will assume that $V(C)=\{u\}$ and that $V(B)=\{w\}$.

Before continuing the proof of the present lemma we need some more notation. Let $s$ be the largest number such that there is a graph $S$ with $|V(S)|=s$, an embedding $\alpha$ from $S$ into $A$, and an embedding $\delta$ from $S$ into $D$. Let $\mathfrak{T}$ be the set of triples of the form $(S, \alpha, \delta)$ such that $S$ is a graph with $|V(S)|=s, \alpha$ an embedding from $S$ into $A$ and $\delta$ an embedding from $S$ into $D$. The triple $(S, \alpha, \delta)$ is $A$-sided if there is a vertex $a \in V(A)-V(\alpha(S))$ which is adjacent to all of the vertices in $V(\alpha(S))$. The triple $(S, \alpha, \delta)$ is $D$ sided if there is a vertex $d \in V(D)-V(\delta(S))$ which is not adjacent to any of the vertices in $V(\delta(S))$. The A-sided triple $(S, \alpha, \delta)$ is minimally $A$ sided if the number of vertices in $V(\delta(S))$ which are adjacent to all vertices in $V(D)-V(\delta(S))$ is minimal over all $A$-sided triples of $\mathfrak{T}$. The $D$-sided triple $(S, \alpha, \delta)$ is minimally $D$-sided if the number of vertices in $V(\alpha(S))$ which are not adjacent to any of the vertices in $V(A)-V(\alpha(S))$ is minimal over all $D$-sided triples of $\mathfrak{T}$.

We associate with each triple $(S, \alpha, \delta) \in \mathfrak{T}$ a set of graphs $\operatorname{amalg}(S, \alpha, \delta)$. The graph $J$ is an element of $\operatorname{amalg}(S, \alpha, \delta)$ if and only if there are two embeddings $\alpha_{0}: A \rightarrow J$ and $\delta_{0}: D \rightarrow J$ such that:

(i) $\alpha_{0} \circ \alpha=\delta_{0} \circ \delta$,

(ii) $V\left(\alpha_{0}(A-\alpha(S))\right) \cap V\left(\delta_{0}(D-\delta(S))\right)=\varnothing$,

(iii) $V(J)=V\left(\alpha_{0}(A)\right) \cup V\left(\delta_{0}(D)\right)$. 


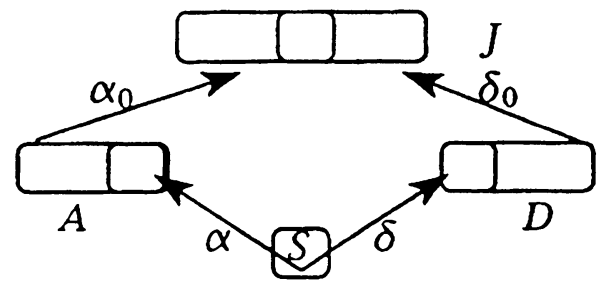

Observe that if $K_{0} \cong K$ and $G_{0} \cong G$ then $\operatorname{Forb}\left(K_{0}, G\right)=\operatorname{Forb}(K, G)$. This means that in proving that the graph $J \in \operatorname{amalg}(S, \alpha, \delta)$ is a graph in Forb $(A+B, C \oplus D)$ we can assume without loss of generality that all of the embeddings $\alpha, \delta, \alpha_{0}$ and $\delta_{0}$ are identity maps. In order to simplify the notation we will make this assumption in such a proof.

We will now prove that if $\mathfrak{T}$ contains an $A$-sided triple then $|V(A)|-s=$ 1 or $|V(D)|-s=1$. Observe first that if $|V(A)|-s=0$ then $A$ can be embedded into $D$ and if $|V(D)|-s=0$ then $D$ can be embedded into $A$. Let $(S, \alpha, \delta)$ be a minimally $A$-sided triple in $\mathfrak{T}$ and assume to the contrary that $|V(A)-V(\alpha(S))|=|V(A)|-s \geq 2$ and that $|V(D)-V(\delta(S))|=|V(D)|-s \geq 2$. Let the graph $J \in \operatorname{amalg}(S, \alpha, \delta)$ be such that each vertex of $A-S$ is not adjacent in $J$ to some vertex of $D-S$. Also, each vertex of $D-S$ is adjacent to some vertex of $A-S$. This can be arranged because $|V(A)|-S \geq 2$ and $|V(D)|-s \geq 2$.

To arrive at a contradiction we will show that $J \in \operatorname{Forb}(A+B, C \oplus D)$. Remember that $|V(B)|=|V(C)|=1,\{w\}=V(B),\{u\}=V(C)$ and let $H=A+B$ and $G=C \oplus D$. Observe that if $\varphi$ is an embedding from $A$ into $J$ then, because of the maximality of $|V(S)|, A-S$ is in the image of $\varphi$. Hence if $\varphi$ is an embedding from $H$ into $J$, then $\varphi(w) \notin V(A-S)$. Also, $\varphi(w) \notin V(D-S)$ because every vertex of $D-S$ is adjacent to some vertex of $A-S$. The vetex $\varphi(w)$ is not an element of $V(S)$ because $(S, \alpha, \delta)$ is $A$-sided and hence there is a vertex of $A-S$ adjacent to all vertices of $S$. This means that there is no embedding from $H$ into $J$. If $\varphi$ is an embedding from $G$ into $J$, then $\varphi(u) \notin V(D-S)$. This is so because the maximality of $S$ implies that $V(D-S) \subseteq V(\varphi(D))$. Also, $\varphi(u) \notin V(A-S)$ because very vertex of $A-S$ is not adjacent to some vertex of $D-S$. This leaves the possibility that $\varphi(u) \in V(S)$. The vertex $\varphi(u)$ of $S$ is then adjacent to all of the vertices in $D-S$ but $\varphi(u)$ is not a vertex of $\varphi(D)$. The triple $(\varphi(D)-(D-S)$, identity, $\left.\varphi^{-1} \mid(\varphi(D)-(D-S))\right) \in \mathfrak{T}$ is $A$-sided because $\varphi(u)$ is adjacent to every vertex of $\varphi(D)-(D-S)$. But $\varphi(D)-(D-S)$ contains fewer vertices adjacent to every vertex of $D-S$ because no vertex of $A-S$ is adjacent to every vertex of $D-S$ and $\varphi(u)$ is not a vertex of $\varphi(D)-(D-S)$. This implies that $(S, \alpha, \delta)$ is not a minimally $A$-sided triple in $\mathfrak{T}$.

We have proven that if $\mathfrak{T}$ contains an $A$-sided triple then $|V(A)|-s=1$ or $|V(D)|-s=1$ and dually that if $\mathfrak{T}$ contains a $D$-sided triple then $|V(A)|-s=1$ or $|V(D)|-s=1$.

Now we will prove that in the case that $\mathfrak{T}$ does not contain an $A$-sided triple nor a $D$-sided triple then also $|V(A)|-s=1$ or $|V(D)|-s=1$ holds. We choose the graph $J$ as above and remember that if $\varphi$ is an embedding from $H$ into $J$ then $\varphi(w) \in V(S)$. This implies that $\left(\varphi(A)-(A-S), \varphi^{-1} \mid(\varphi(A)-(A-S))\right.$, identity) would be a $D$-sided triple in $\mathfrak{T}$. Dually, there is no embedding $\varphi$ 
from $G$ into $J$. Hence we have proven that $|V(A)|-s=1$ or that dually $|V(D)|-s=1$. Assume that $|V(D)|-s=1$.

In order to complete the proof of the present lemma it suffices to see that there is a $D$-sided triple $(S, \alpha, \delta)$. This is so because we have already shown that $|V(D)-V(\delta(S))|=1$ and it follows from the definition of $D$-sided triple that then the vertex in $V(D)-V(\delta(S))$ is not adjacent to any vertex in $V(\delta(S))$. In order to prove that there exists such a $D$-sided triple choose the triple $(S, \alpha, \delta) \in \mathfrak{T}$ so that the number of vertices of $\delta(S)$ adjacent to the single vertex of $D-\delta(S)$ is minimal. Denote the single vertex of $D-\delta(S)$ by $v$. We wish to prove that $v$ is an isolated vertex of $D$. Assume not. Let then the graph $J \in \operatorname{amalg}(S, \alpha, \delta)$ be such that no vertex of $A-S$ is adjacent to $v$ in $J$. (For ease of argument we assume again that the embeddings $\alpha, \delta$, $\alpha_{0}, \delta_{0}$ are identity maps.) We will prove that $J \in \operatorname{Forb}(G, H)$.

If $\varphi$ would be an embedding from $H$ into $J$, then $\varphi(w) \notin V(A-S)$ as above and $\varphi(w) \neq v$, otherwise $v$ would be an isolated vertex of $D$. If $\varphi(w) \in V(S)$, the triple $\left(\varphi(A)-(A-S), \varphi^{-1} \mid(\varphi(A)-(A-S))\right.$, idetnity) $\in \mathfrak{T}$ would be $D$-sided. If $\varphi$ would be an embedding from $G$ into $J$ observe first that $v \in V(\varphi(D))$ because there is no embedding from $D$ into $A$. This implies immediately that $\varphi(u) \neq v$. It is also true that $\varphi(u) \notin A-S$ because the vertices of $A-S$ are not adjacent to $v \in V(\varphi(D))$. If $\varphi(u) \in V(S)$, $\varphi(u)$ would have to be adjacent to $v \in V(\varphi(D))$. Hence, and also because there are no adjacencies between $v$ and vertices of $A-S$, the triple $(\varphi(D)-$ $v$, identity, $\left.\varphi^{-1}\right) \in \mathfrak{T}$ would disprove the minimality of the degree of $v$ in $D$. (The vertex $\varphi^{-1}(v)$ has smaller degree in $D$ than the vertex $v$. To see this let $X$ be the set of vertices adjacent to $v$ and $Y$ be the set of vertices adjacent to $\varphi^{-1}(v)$. Then the vertex $\varphi(u) \in X-Y$ is adjacent to $v$ but no vertex in $Y-X$ is adjacent to $\varphi^{-1}(v)$.) We conclude that $D$ contains the isolated vertex $v$ and that there is an embedding $\delta$ from $D-v$ into $A$.

Let $A$ and $M$ be graphs and $u, v$, and $w$ be three vertices not in $A$ or $M$. $D, H$ and $G$ are given by $D=v+M, G=u \oplus D$ and $H=w+A$. Most of the following lemmas will discuss this situation. We will always assume that $G \in \operatorname{Forb}(H), H \in \operatorname{Forb}(G)$ and $A \in \operatorname{Forb}(D)$.

Lemma 9. Let $A$ and $M$ be graphs with $|V(A)|>|V(M)|=m>1$ such that $A \nsubseteq P_{2}, M$ consists of a set of pairwise not adjacent vertices, and $A$ can be embedded into $P_{2 m}$, the path on $2 m+1$ vertices. Let $u, v$, and $w$ be three vertices not in $A$ or $M . D, H$, and $G$ are given by $D=v+M, G=u \oplus D$, and $H=w+A$. Then there is a graph $J \in \operatorname{Forb}(G, H)$ into which both graphs $A$ and $D$ can be embedded.

Proof. We first consider the case that $m \geq 3$. The graph $A$ does not contain a vertex with degree $\geq 3$. Let $J$ be the graph with vertex set $V(J)=\left\{x_{i}: i \in\right.$ $m+1\} \cup\{y, z\}$ and edge set $E(J)=\left\{\left\{y, x_{i}\right\}: i \in m+1\right.$ and $\left.i \neq m\right\} \cup$ $\left\{z, x_{i}\right\}: i \in m$. The graph $J$ is connected and $D$ can be embedded into $J$. The graph $G$ cannot be embedded into $J$ because $J$ does not contain a vertex of degree $m+1$. We prove next that $N$ cannot be embedded into $J$. Because $|V(H)| \geq m+2$ and $|V(J)|=m+3$ it follows that if the graph $H$ can be embedded into $J$ then $H$ is either isomorphic to $J$ or $|V(H)|=m+2$. The graph $H$ is not isomorphic to $J$ because $J$ does not contain an isolated vertex. Hence if $H$ can be embedded into $J$ then $H$ is isomorphic to $J-r$ for some 
vertex $r \in V(J)$ such that $J-r$ has an isolated vertex. This implies that $r=y$ or $r=z$ and we can assume without loss of generality that $r=y$. The only isolated vertex of $J-y$ is $x_{0}$ and hence $A$ is isomorphic to $J-y-x_{0}$, which is not possible because $z$ as a vertex of $J-y-x_{0}$ has degree $m \geq 3$.

For the remaining cases $m=2$ and $G$ is a star with four vertices. Hence $D$ consists of three isolated vertices and $A$ can be embedded into the path $P_{4}$ having five vertices. We will prove that there is a graph $J \in \operatorname{Forb}(G, H)$ into which both graphs $A$ and $D$ can be embedded. If $A$ contains three pairwise nonadjacent vertices then $D$ can be embedded into $A$ and hence we can put $J=A$. We can now assume that $A$ does not contain three pairwise nonadjacent vertices. Hence $A$ contains at least one edge, otherwise $m=2 \geq|V(A)|$. If $A$ contains exactly one edge and no other vertex then $m=2 \geq|V(A)|$. Hence $A$ contains only one vertex not in that edge and we may assume that $A=K_{2}+K_{1}$. Put $J=P_{2}+K_{1}$. If $A$ contains exactly two edges which are nonadjacent we may assume that $A=K_{2}+K_{2}$. Put $J=P_{2}+K_{2}$. If $A$ contains exactly two edges which are adjacent then $A \cong P_{2}$ contrary to our assumptions. The graph $A \neq P_{4}$, because $P_{4}$ contains three pairwise nonadjacent vertices. Hence if $A$ contains at least three edges then $A \cong P_{3}$. In this case put $J=C_{6}$, the circuit with six vertices.

Lemma 10. Let $A$ and $M$ be graphs with $|V(A)|>|V(M)|>1$ such that, if $M$ consists of a set of pairwise not adjacent vertices, then $A \not P_{2}$. Let $u$, $v$, and $w$ be three vertices not in $A$ or $M$. The graphs $D, H$, and $G$ are given by $D=v+M, G=u \oplus D$ and $H=w+A$. Then, if there is no graph $J \in \operatorname{Forb}(G, H)$ into which both graphs $A$ and $D$ can be embedded, there is $a$ connected graph $R \in \operatorname{Forb}(G)$ into which $D$ can be embedded but $A$ cannot be embedded.

Proof. Observe that if a graph $R \in \operatorname{Forb}(G, H)$ is connected and $D$ can be embedded into $R$ then $R \in \operatorname{Forb}(G)$ is a connected graph into which $D$ can be embedded but $A$ cannot be embedded. Otherwise $J=R \in \operatorname{Forb}(G, H)$ would be a graph into which both graphs $A$ and $D$ can be embedded. Hence we have to find a connected graph $R \in \operatorname{Forb}(G, H)$ into which $D$ can be embedded. Assume first that $G$ is not a star. In that case, let $R$ be the graph with vertex set $V(R)=V(G)$. The edges of $R$ are such that $R \mid V(D)=D$ and such that $u$ is adjacent to exactly one vertex of every connected component of $D$. If $G$ is not a star we have removed edges from $G$ to obtain $R$. Hence $G$ cannot be embedded into $R$. The graph $R$ is connected and $D$ can be embedded into $R$. It remains to prove that $H$ cannot be embedded into $R$. We deduce from $|V(H)|=|V(A)|+1 \geq|V(M)|+1+1=|V(G)|=|V(R)|$ implies that if $H$ can be embedded into $R$, then $H$ and $R$ are isomorphic; this is impossible because $H$ has an isolated vertex, and $R$ is connected.

If $G$ is a star with $m=|V(M)| \geq 2$ we consider the path $P_{2 m}$ with $2 m+1$ vertices. The path $P_{2 m} \in \operatorname{Forb}(G)$ is connected and $D$ can be embedded into $P_{2 m}$. It follows now from Lemma 9 that $A$ cannot be embedded into $P_{2 m}$.

Lemma 11. Let $A$ and $M$ be graphs with $|V(A)|>|V(M)|>1$ and $R \in$ Forb $(G)$ be a connected graph. Let $u, v$, and $w$ be three vertices not in $A$ or $M$. The graphs $D, H$, and $G$ are given by $D=v+M, G=u \oplus D$ and $H=w+A$ and assume that none of the two graphs $G$ and $H$ can be embedded into the other. If there is no graph $J \in \operatorname{Forb}(G, H)$ into which both 
graphs $A$ and $D$ can be embedded, if $D$ can be embedded into $R$ but $A$ cannot be embedded into $R$, and if $\operatorname{Forb}(G, H)$ is Ramsey, then the graph $A$ is an $M$-tree.

Proof. Note that $D$ cannot be embedded into $A$, otherwise

$$
J=A \in \operatorname{Forb}(G, H)
$$

embeds both graphs $D$ and $A$. The graph $A$ is in $\operatorname{Forb}(G)$ because $A$ can be embedded into $H$. The graph $A$ is in $\operatorname{Forb}(H)$ because $|V(A)|<|V(H)|$.

We prove first that if $A=A_{1}+A_{2}$ is disconnected with $A_{1}$ a connected component of $A$ with a minimal number of vertices, then there is no embedding from $A$ into $M+A_{1}$. Assume for a contradiction, that there is an embedding $\beta$ from $A$ into $M+A_{1}$. The graph $A_{1}$ is a smallest connected component of $A$. Hence, only a component of $A$ isomorphic to $A_{1}$ can be mapped by $\beta$ into $A_{1}$. This implies that $\beta$ embeds a subgraph of $A$ which is isomorphic to $A_{2}$ into $M$, and hence $M$ embeds $A_{2}$. Let $A^{\prime}=w+A_{1}$ and $B^{\prime}=A_{2}$, then $\operatorname{Forb}(H, G)=\operatorname{Forb}\left(A^{\prime}+B^{\prime}, u \oplus D\right)$. We deduce from Lemma 8, that either $B^{\prime}=A_{2}$ has only one vertex or there is a graph $J \in \operatorname{Forb}(G, H)$ such that both graphs $A^{\prime}$ and $D$ can be embedded into $J$. If there is such a graph $J \in \operatorname{Forb}(G, H)$, then also $A_{2}$ can be embedded into $J$ because $D$ embeds $M$ which in turn embeds $A_{2}$ and $u$ can be mapped to any vertex. Hence, $J$ embeds every connected component of $H$ and every dual component of $G$. Applying Lemma 2 and the fact that $\operatorname{Forb}(G, H)$ has the Ramsey property, we arrive at the contradiction that $H$ or $G$ in $\operatorname{Forb}(H, G)$. Hence $A_{2}$ has only one vertex and $A_{1}$ is a smallest connected component of $A$, hence $A_{1}$ too has only one vertex. But this is not possible because $|V(A)|>|V(M)|>1$. We conclude that there is no embedding from $A$ into $M+A_{1}$.

We prove next that the graph $A$ is connected. Assume to the contrary that $A=A_{1}+A_{2}$ where $A_{1}$ is a connected component of $A$ with a minimal number of vertices. We can assume without loss of generality that $V(A) \cap V(M)=\varnothing$. Let $J=A_{1}+\left(A_{2} \oplus M\right)$. We will prove that $J \in \operatorname{Forb}(G, H)$. This is contrary to the assumptions, because it embeds $D$ and $A$. Hence we can then conclude that $A$ is connected.

Assume that there is an embedding $\alpha$ from $G$ into $J$. The graph $G$ is connected and hence $\alpha$ maps $G$ into $A_{1}$ or $\alpha$ maps $G$ into $A_{2} \oplus M$. Because the graph $G$ cannot be embedded into $A_{1}$, otherwise $D$ could be embedded into $A_{1}$ and hence into $A$, we conclude that $\alpha$ embeds $G$ into $A_{2} \oplus M$. If $\alpha(v) \in V(M)$ then $\alpha$ cannot embed any vertex of $M$ into $A_{2}$, because $v$ is not adjacent to any vertex in $M$. The embedding $\alpha$ is a one to one function and hence it cannot embed $M$ together with $v$ into $M$. If $\alpha(v) \in V\left(A_{2}\right)$ then $\alpha$ embeds every vertex of $M$ into $A_{2}$ and hence $\alpha$ would embed $D$ into $A$. Therefore $J \in \operatorname{Forb}(G)$. Assume next that $\alpha$ is an embedding from $H$ into $J$. If $\alpha$ maps $w$ into $A_{2}$, then $\alpha$ embeds $A$ into $\left(A_{2}-\alpha(w)\right)+A_{1}$, which is not possible because $A$ contains more vertices than there are in the graph $\left(A_{2}-\alpha(w)\right)+A_{1}$. If $\alpha$ maps $w$ into $M$, then $\alpha$ embeds $A$ into $M+A_{1}$ which by the previous paragraph is not possible. Hence $\alpha$ maps $w$ into $A_{1}$. The graph $A_{1}$ is a smallest connected component of $A$ and hence $\alpha$ embeds every connected component of $A$ into $A_{2} \oplus M$. This means that $\alpha$ embeds $A$ into $A_{2} \oplus M$. We will argue that this is impossible. If $\alpha$ maps any vertex of $A_{1}$ into $M$ then $\alpha$ embeds $A_{2}$ into $M$, hence $A_{1}$ into $M$ as well and 
hence $A$ into $D$. If $\alpha$ maps any vertex of $A_{1}$ into $A_{2}$ then $\alpha$ embeds $A_{2}$ into $A_{2}$, and hence $A_{1}$ into $A_{2}$ as well. We finally conclude that the graph $A$ is connected.

Let $z \in V(R)$ be a vertex. Assume that there are $r$ subsets of $V(R)$ which are attached to $z$. Let $H$ be a $|V(M)|$-uniform hypergraph with chromatic number larger than $r$ and girth larger than $|V(A)|+5$. Let $L_{1}$ be a graph constructed from the hypergraph $H$ by embedding $M$ into the edges of $H$ and $L=z_{0} \oplus L_{1}$. Then according to Lemma 5 , the graph $L \in \operatorname{Forb}(G)$ and according to Lemma 6 , and the graphs $L$ and $R$ cannot be disjointly amalgamated on $z_{0} \simeq z$. The graph $G$ is connected and because $L \in \operatorname{Forb}(G)$ and $R \in \operatorname{Forb}(G)$ also $L+R \in \operatorname{Forb}(G)$. If $L+R \notin \mathscr{N}_{G}$, that is if $L+R$ is chromatically unbounded, then by the corollary to Theorem 5 , the graph $L$ couid be disjointly amalgamated with $R$ on $z \simeq z_{0}$. We conclude that $L+R \in \mathscr{N}_{G}$. There must be an embedding from $H=w+A$ into $L+R$ because otherwise $L+R \in \operatorname{Forb}(G, H)$ which is Ramsey and hence we would obtain $L+R \notin \mathscr{N}_{G}$ which we just proved to be impossible. In particular there is then an embedding from $A$ into $L+R$. The graph $A$ is connected and cannot be embedded into $R$. Hence, there is an embedding $\alpha$ from $A$ into $L$. We will identify $A$ with the image of $\alpha$. Remember that for every hyperedge $E$ of $H$ there exists an embedding $\varphi_{E}$ from $L \mid E$ to $M$. Let $\mathfrak{S}_{1}=\left\{V(A) \cap \varphi_{E}^{-1}(V(M)): E\right.$ is a hyperedge of $H$ \}. Then $\mathfrak{S}_{1}$ satisfies:

(i) For every $s \in \mathfrak{S}_{1}$ there is an embedding from $A \mid S$ into $M$.

(ii) The hypergraph with vertex set $V(A)$ and edge set $S$ contains no circuits.

Observe also that if $a, b \in V(A)$ are two nonadjacent vertices then there is an $S \in \mathfrak{S}_{1}$ with $a, b \in S$. Hence, the set $\mathfrak{S}=\{S \subseteq V(A): A \mid S$ is a dual block of $A\}$ has the property that for each $S \in \mathfrak{S}$ there is a hyperedge $E$ of $H$ such that $S \subseteq E$. This means that for every $s \in \mathfrak{S}_{1}$ there is an embedding from $A \mid S$ into $M$. Hence $A$ is an $M$-tree. (In other words the graph $L$ is obviously an $M$-tree which contains the graph $A$ as an induced subgraph. The assertion of the lemma follows because every induced subgraph of an $M$-tree is an $M$-tree.)

\subsection{The fiRst MAIN LEMMA FOR CASE 2}

Lemma 12. Let $A$ and $M$ with $|V(A)|>|V(M)|>1$ be graphs such that there is an embedding from $M$ into $A$. There also exists an embedding $\delta$ from $M-p$ into $A$ for some vertex $p \in V(M)$ and there is a vertex $a \in V(A-\delta(M-p))$ such that no vertex of $\delta(M-p)$ is adjacent to the vertex $a$. For $u, v$, and $w$, three vertices not in $A$ or $M$, the graphs $D, H$, and $G$ are given by $D=v+M, G=u \oplus D$, and $H=w+A$. The graphs $A$ and $M$ are elements of $\operatorname{Forb}(G, H)$ and $A$ is an $M$-tree. Then either $M$ is complete, or there exists a graph $J \in \operatorname{Forb}(G, H)$ such that both graphs $A$ and $D$ can be embedded into $J$, or $G \cong K_{1} \oplus\left(K_{1}+K_{1}+K_{1}\right)$ and $H \cong K_{1}+P_{2}$.

Proof. In order to simplify the notation we will assume that the embedding $\delta$ is the identity function. $M-p$ is then an induced subgraph of $A$ and the vertex $a$ is not adjacent to any of the vertices of $M-p$. A vertex $x \in V(A)$ is called central if there exists a vertex $y \in V(M)$ and an embedding $\alpha$ from $M-y$ into $A-x$ such that $x$ is not adjacent to any of the vertices of $\alpha(M-y)$. Observe that the vertex $a$ is central and that we may assume that $a$ is a central vertex 
having minimal degree among all central vertices of $A$. If $D$ can be embedded into $A$ then the graph $J=A$ is in $\operatorname{Forb}(G, H)$ such that both graphs $A$ and $D$ can be embedded into $J$. Hence we can assume that there is no embedding from $D$ into $A$.

Let $m$ be the largest number of vertices in a dual block of $M$ and $C$ a dual block of $M$ with $|V(C)|=m$ which has a minimal number $e$ of edges. We will assume that $M$ is not complete and hence in particular that $m>1$ holds. Put $\mathfrak{S}=\{S \subseteq V(A): A \mid S$ is a dual block of $A\}$. Observe that for every embedding $\delta$ from $C$ into $A$ there exists one and only one element $S \in \mathfrak{S}$ such that $V(\delta(C)) \subseteq S$. Because $A$ is an $M$-tree and because $C$ is a dual block of $M$ with a maximal number of vertices, $\delta(C)$ is a dual block of $A$. This implies that the hypergraph formed by the copies of $C$ in $A$ does not contain a circuit. No vertex of $M-p$ is adjacent to the vertex $a$. Hence every vertex of $M-p$ is in some dual block of $A$ which contains $a$.

Let $\mathscr{B}$ be the set of dual blocks of $A$ which contain $a$. Let $X$ be a dual block of $M$. Observe that either the vertex $p \in V(X)$ or $p \notin V(X)$. If $p \notin V(X)$ then there exists a unique dual block $B(X)=B \in \mathscr{B}$ such that $\mathscr{V}(X) \subseteq V(B)$. If $X$ is a dual block of $M$ which contains the vertex $p$, then $\bar{X}=\bar{M} \mid(V(X-p) \cup\{p\})$ is two-connected. The vertex $a$ is not adjacent to any of the vertices of $X-p$ and hence also $\bar{A} \mid(V(X-p) \cup\{a\})$ is two-connected. This means that there is also in this case a unique dual block $B(X) \in \mathscr{B}$ such that $V(x-p) \subseteq V(B)$ holds. If $X$ is a dual block of $M$ which contains $m$ vertices and the vertex $p \notin V(X)$, then $V(X) \subseteq V(B(X))$. Now then, $|V(B(X))| \geq|V(X)|+1=m+1$ because $B(X)$ contains the vertex $a$, which is not a vertex of $X$. This is not possible because the graph $A$ is an $M$ tree and hence there is an embedding from $B(X)$ into $M$ which implies that $|V(B(X))| \leq m$. We conclude that if $X$ is a dual block of $M$ which contains $m$ vertices, then $p \in V(X), V(B(X))=V(X-p) \cup\{a\}$ and $B(X)$ is isomorphic to some dual block of $M$ containing $m$ vertices. In particular it follows that $p \in$ $V(C)$ holds and by the same token all dual blocks of $M$ which are isomorphic to $C$ share the vertex $p$. Also because the vertex $a$ is not adjacent to any of the vertices in $X-p$, the number of edges of $B(X)$ is less than or equal to the number of edges of $X$. If the vertex $p$ is adjacent to any of the vertices in $X-p$, then the number of edges of $B(X)$ is strictly smaller than the number of edges of $X$. From the minimality of the number of edges of $C$ amongst all $m$-element dual blocks of $M$, it follows that the vertex $p$ is not adjacent to any of the vertices in $C-p$. Observe that therefore $C$ is isomorphic to $A \mid(V(C-p) \cup\{a\})$, that is $C$ is isomorphic to $B(C)$. Note that the above argument did not use the fact that $a$ has minimal degree amongst all of the central vertices of $A$. We have therefore established the following:

Proposition 1. If $x$ is a central vertex of $A$ and the vertex $y \in V(M)$ and the embedding $\delta: M-y \rightarrow A-x$ are such that no vertex of $\delta(M-y)$ is adjacent to $x$, then $y \in V(C)$ and $y$ is not adjacent to any vertex of $C-y$. Also, if $F=A \mid(V(\delta(C-y)) \cup\{x\})$, then $F$ is an isomorphic copy of $C$ in $A$.

In particular, if $m=2$, then any two vertices of $M-p$ are adjacent. Otherwise if the vertices $x, y \in V(M-p)$ are not adjacent, then $A \mid\{a, x, y\} \cong \overline{K_{3}}$ and hence $A$ and therefore $M$ would have a block containing three vertices. This means that if $m=2$ then $\bar{C} \cong K_{2}$ and $\bar{M}$ is the sum of a possibly empty 
set $T$ of isolated vertices with a star and $\bar{A}$ is a forest. We denote the set of endpoints of this star in $\bar{M}$ by $S$ and note that $p$ is the center of this star. If we put $l=|S|$ then the degree of $p$ in $\bar{M}$ is $l$. Let $R=S \cup T \cup\{a\}$. Note that $S \cup T$ induces a complete subgraph of $M$ and hence that $A \mid(S \cup T)=M-p$ is a complete graph.

Case 1: $|V(A)|=|V(M)|+1$. We consider the case $|V(M)|=2$ first. If $M$ is not complete then $M$ consists of two isolated vertices and $G \cong K_{1} \oplus$ $\left(K_{1}+K_{1}+K_{1}\right)$ is a star having four vertices. Clearly $m=2$ holds, and because $\bar{A}$ is a forest, not complete, and $|V(A)|=3$, either $A \cong P_{2}$ or $\bar{A} \cong P_{2}$. If $A \cong P_{2}$ then we have arrived at one of the possible conclusions of Lemma 12 and so assume that $\bar{A} \cong P_{2}$. Let $J=P_{2}+K_{1}$. It is easy to see that both graphs $D$ and $A$ can be embedded into $J$ and that $J \in \operatorname{Forb}(G, H)$. For the remainder of Case 1 we will assume that $|V(M)| \geq 3$.

Assume first that the vertex $z \in\{z\}=V(A)-V(M-p)-\{a\}$ is not adjacent to all the other vertices of $A$. Observe that no vertex in $A-z$ is adjacent to all the other vertices of $A$ because $a$ is not adjacent to any vertex in $V(M-p)=V(A-z-a)$. Let $b \notin V(A)$ be a vertex and $\beta$ an embedding from $M$ into $A$. The graph $J$ is defined with vertex set $V(J)=V(A) \cup\{b\}$ and edge set such that $J \mid V(A)=A$ and $b$ is an endpoint of $J$ adjacent only to the vertex $x \in V(A)-V(\beta(M))=\{x\}$. Clearly, $A$ can be embedded into $J$ and $J-x$ is isomorphic to $D$. The graph $G$ cannot be embedded into $J$ because otherwise $G$ would be isomorphic to $J$ but no vertex of $J$ is adjacent to all the other vertices of $J$. There is no embedding $\alpha$ from $H$ into $J$ because $H$ would be isomorphic to $J$ with $\alpha(w)$ an isolated vertex of $J$. But neither $b$ nor $x$ are isolated vertices of $J$ and hence $\alpha(w) \in V(A)-x$ and $A$ is isomorphic to $J-\alpha(w)$. This is not possible because $J-\alpha(w)$ has one more edge than $A$. For the remainder of Case 1 we will assume that the vertex $z \in\{z\}=V(A)-V(M-p)-\{a\}$ is adjacent to all the other vertices of $A$.

Assume next that there is a vertex $y \in V(A-z-a)$ such that $A-y$ is isomorphic to $M$. Let $b$ be a vertex not in $A$. Let $J$ be the graph with vertex set $V(J)=V(A) \cup\{b\}$. The edges of $J$ are such that $J \mid V(A)=A$ and $b$ is an endpoint of $J$ only adjacent to $y$. Clearly, $A$ can be embedded into $J$ and $J \mid(V(A-y) \cup\{b\})$ is isomorphic to $D$. The graph $G$ cannot be embedded into $J$, because no vertex of $J$ has degree $|V(M)|+1$, while the vertex $u \in V(G)$ does have degree $|V(M)|+1$. (The vertex $y$ is not adjacent to $a$ and every other vertex $A$ is not adjacent to $b$.) The graph $J$ has the same number of vertices as $H$ and hence if $H$ could be embedded into $J, H$ and $J$ would be isomorphic. But $J$ is connected, because $z$ is adjacent to all the other vertices of $A$, while $H$ is not connected. Hence $J \in \operatorname{Forb}(G, H)$.

Next we consider the situation that the vertex $p$ is an isolated vertex of $M$. Let $b$ be a vertex not in $V(A) \cup\{p\}$. Let $J$ be the graph with vertex set $V(J)=V(A) \cup\{b, p\}$. The edges of $J$ are such that $J \mid V(A)=A$ and $b$ and $p$ are endpoints of $J$ both adjacent only to $a$. Observe that the graph $A$ can be embedded into $J$ and $J \mid(V(J)-z-a)$ is isomorphic to $D$. The graph $G$ cannot be embedded into $J$ because no vertex of $J$ has a degree larger than or equal to $|V(M)|+1$ while $u$ has degree $|V(M)|+1$. The vertex $a$ has degree three and if $|V(M)|+1=3$, then $|V(M)|=2$.

Next we prove that $J \in \operatorname{Forb}(H)$. Assume to the contrary that $\alpha$ is an 
embedding from $H$ into $J$. If $\alpha(w)=p$ then $A$ would have to be isomorphic to $J-p-a$. But in $J-p-a$ the vertex $b$ is isolated while $A$ does not contain an isolated vertex. Similarly $\alpha(w) \neq b$. The degree of $z$ is larger than one and hence $\alpha(w) \neq z$. (The vertex $z$ is adjacent to all other vertices of $A$.) Similarly, the degree of $a$ is three and hence $\alpha(w) \neq a$. This leaves $\alpha(w) \in V(A-a-z)$. Then $\alpha(A)$ is equal to $J-\alpha(w)-z$. But if $|V(M)|>2$ then $A$ has at least one more edge than $J-\alpha(w)-z$. Hence $J \in \operatorname{Forb}(G, H)$.

We know that there is an embedding $\alpha$ from $M$ into $A$. If $V(\alpha(M))=$ $V(M-p) \cup\{a\}$ then, because the vertex $a$ is not adjacent to any vertex of $M-p$, also $p$ cannot be adjacent to any vertex of $M-p$, otherwise $M$ would contain more edges than $A \mid(V(M-p) \cup\{a\})=\alpha(M)$. This means that the vertex $p$ is an isolated vertex of $M$, a situation which we have discussed in the previous paragraph. If $\alpha(M)$ contains both vertices $a$ and $z \in\{z\}=V(A)-$ $V(M-p)-\{a\}$, then $y \in\{y\}=V(A)-V(\alpha(M))$ is an element of $V(A-z-a)$ such that $A-y$ is isomorphic to $M$. This too is then a situation which we have already discussed. Hence we may assume that $V(\alpha(M))=V(M-p) \cup\{z\}$. This implies that the vertex $p$ is adjacent to every other vertex of $M-p$, because we have assumed that the vertex $z$ is adjacent to every vertex of $M-p$. But this is in contradiction to Proposition 1 according to which the vertex $p$ is not adjacent to any of the vertices in $C-p$.

Case 2: $m=2$. Let $P=\{x \in V(A)-R: \exists y \in S$ such that $x$ is not adjacent to $y$ \}. Note that in $\bar{A}$, the set $P$ is the set of neighbors of the vertices in $S$ which are not in $R$, that is the neighbors of $S$ which are not equal to the vertex $a$. Let $\Delta: V(A) \rightarrow$ red, blue $\}$ be a colouring of $A$ with two colours such that for all $x \in R \cup P, \Delta(x)=$ red and if $x$ and $y$ are two nonadjacent vertices of $A$ with $\Delta(x)=\Delta(y)$, then $\{x, y\} \subseteq R \cup P$. Amongst all of such colourings $\Delta$ we first minimize the difference between the number of red and blue vertices of $V(A)-R-P$ and then maximize the number of blue vertices of $V(A)-R-P$. Let $b \notin V(A) \cup\{p\}$ be a vertex and $J$ a graph with vertex set $V(J)=V(A) \cup\{p\} \cup\{b\}$. The edge set of $J$ is such that $J \mid V(A)=A$ and $J \mid V(M)=M$. The vertex $x \in V(J)$ is adjacent to $b$ if and only if $x \in V(A)-R-P$ and $\Delta(x)=$ blue, or $x \in P \cup\{a\}$. The vertex $x \in V(J)$ is adjacent to $p$ if and only if $x \in V(A)-R-P$ and $\Delta(x)=$ red, or $x \in P \cup T \cup\{a\}$. Note that $p$ is not adjacent to $b$, the set of vertices adjacent to both vertices $p$ and $b$ is $P \cup\{a\}$, the set of vertices of $V(A)$ not adjacent to any one of the vertices $p$ and $b$ is $S$, and if the vertices $x$ and $y$ of $A$ are not adjacent then at least one of $x$ and $y$ is adjacent to $p$ and one of $x$ and $y$ is adjacent to $b$. (Every vertex in $S$ which is not adjacent to either $p$ or $b$ is only not adjacent to such vertices of $A$ which are adjacent to both vertices $p$ and $b$.) It follows that the graph $A$ can be embedded into $J$ and $J \mid(S \cup T \cup\{p\} \cup\{b\})$ is isomorphic to $D$.

We will prove that $J \in \operatorname{Forb}(G)$. Assume to the contrary that $\alpha$ is an embedding from $G$ into $J$. If $l=1$ then $M$ contains two nonadjacent vertices $p$ and some other vertex, say $q$, the graph $G$ contains as an induced subgraph a star having four vertices and $D$ contains the three pairwise nonadjacent vertices $p, q$, and $v$. The graph $A$ is a forest and hence at most two of the three vertices $\alpha(v), \alpha(p), \alpha(q)$, say $\alpha(p)$ and $\alpha(q)$, are in $A$. But then one of $\alpha(p)$ and $\alpha(q)$ is adjacent to $p$ and one of $\alpha(p)$ and $\alpha(q)$ is adjacent to $b$. 
Hence only one of the three vertices $\alpha(v), \alpha(p), \alpha(q)$, say $\alpha(q)$, is in $A$. Then, without loss of generality $\alpha(v)=b$ and $\alpha(p)=p$. The only vertices of $J$ not adjacent to either of the vertices $b$ and $p$ are the vertices of $S$. Hence $\alpha(q) \in S=\{\alpha(q)\}$. The only vertices of $J$ adjacent to both vertices $b$ and $p$ are the vertices in $P \cup\{a\}$. Hence $\alpha(u) \in P \cup\{a\}$. But this is impossible for no vertex of $P \cup\{a\}$ is adjacent to $\alpha(q)$ because $l=|S|=1$. We assume now that $l>1$.

Remember again that $\bar{A}$ is a forest. Hence if $\alpha(v) \in V(A)$ and $\alpha(p) \in V(A)$ then no vertex of $S$ can be in $A$. This means that $l=2$ and both of the vertices in $S$ are mapped by $\alpha$ into the set $\{b, p\}$. But this is impossible because at least one vertex of any two nonadjacent vertices of $A$ is adjacent to $p$ or $b$ and $\alpha(p)$ and $\alpha(v)$ are not adjacent. Hence if $\alpha(v) \in V(A)$, then $\alpha(p)=b$ or $\alpha(p)=p$. For $x \in S, \alpha(p)$ is not adjacent to $\alpha(v)$ and not adjacent to $\alpha(x)$. Also $\alpha(v)$ and $\alpha(x)$ are not adjacent. Both $p$ and $b$ are adjacent to at least one vertex of every pair of nonadjacent vertices of $A$. Hence $\alpha(x)=\{p, b\}$. We conclude that $l=1$ and hence using the previous paragraph that $\alpha(v) \notin V(A)$. Similarly then $\alpha(p) \notin V(A)$. Hence $\{\alpha(v), a(p)\}=\{b, p\}$ holds. The only vertices of $J$ not adjacent to either vertex $v$ or $b$ are the vertices of $S$. Hence $\alpha(S)=S$. Then $\alpha(u)$ is adjacent to both vertices $p$ and $b$ and hence $\alpha(u) \in P \cup\{a\}$. This establishes the desired contradiction because every vertex in $P \cup\{a\}$ is not adjacent to at least one vertex of $S$.

We will prove next that $J \in \operatorname{Forb}(H)$. Assume to the contrary that $\alpha$ is an embedding from $H$ into $J$. The graph $H$ is isomorphic to $J-x$ for some vertex $x \in V(J)$ and $H$ contains the isolated vertex $w$ and hence in $J$ the vertex $\alpha(w)$ has degree at most one and is only adjacent to the vertex $x$. We will investigate all of the possible values of $\alpha(w)$ in $V(J)$.

If $\alpha(w)=p$ then $P=T=\varnothing$ and no vertex of $A-a$ is adjacent to $p$. The fact that $T=\varnothing$ means that $p$ is an isolated vertex of $M$. Let then $N$ be the set of vertices in $A-R$ which are not adjacent to the vertex $a$. All vertices in $N$ are coloured blue by $\Delta$. Because the vertex $p$ has degree one in $J$, no vertex of $A-R-N$ is coloured red by $\Delta$. Hence if $N=\varnothing$, then $A \mid(S \cup\{a\})$ is a connected component of $\bar{A}$. If there is no red vertex in $V(A)-S-a$, then $V(A)-R$ contains at most one vertex and hence $|V(A)| \leq|V(M)|+1$. If $N \neq \varnothing$, then $A-R-N=\varnothing$ because the vertices in $N$ are coloured blue and hence by minimizing the difference between the red and the blue vertices of $A-R-P$, at least one vertex of $A-R-N$ would have to be coloured red and thus be adjacent to the vertex $p$. Note that if $\alpha(w)=p$, then $A \cong J-p-a$. We obtain $J-p-a$ from $A$, by removing the vertex $a$ and adding the vertex $b$. But $a$ is not adjacent to $|S|+|N|$ vertices while the vertex $b$ is not adjacent to $|S|$ vertices and hence $A$ has fewer edges than $J-p-a$. Hence $\alpha(w) \neq p$.

If the vertex $b$ is only adjacent to the vertex $a$, then $V(A)-R=\varnothing$, because of the maximizing condition for the number of blue vertices, and we would have that $|V(A)|=|V(M)|$ holds, which is contrary to the assumption that $|V(A)|>|V(M)|$. Hence $\alpha(w) \neq b$.

The vertex $a$ has degree at least two in $J$. If there are at least three vertices in $A$ which are not adjacent to $a$ then every vertex of $A$ has at least degree two. (The graph $\bar{A}$ is a tree and hence any three such vertices form a triangle in $A$ and every other vertex of $A-a$ is not adjacent to at most one of those 
three vertices.) Therefore there are at most two vertices of $A$ not adjacent to $a$. If there are exactly two such vertices $z$ and $y$ then either both of them or exactly one of them is an element of $S$ because the vertices $s$ and $y$ are adjacent and every vertex not in $S$ is adjacent to $p$ or $b$.

Assume that both vertices $s$ and $y$ are elements of $S$; then $V(M-p)=$ $\{z, y\}$. Hence $M-p$ consists of two adjacent vertices and hence every vertex which is in $A$ not adjacent to at least two other vertices is central. (Because $\bar{A}$ is a tree such two vertices must be adjacent.) If in $V(A-z-y)$ there is a vertex adjacent to $z$ and a vertex (possibly the same) adjacent to $y$, then every vertex of $A$ has in $J$ a degree of at least two. We may therefore assume that no vertex of $V(A-y-z)$ is adjacent to $z$. If there are at least two such vertices in $A-a-y$, then $z$ is central but has smaller degree than $a$. This implies that $\bar{A}$, and therefore also $A$, is a path of length three. The only vertex of $J$ with degree less than two is $z$. Hence $A$ must be isomorphic to $J-z-y$. But $J-z-y$ is not a path of length three because $a$ is adjacent to three vertices of this graph.

If only one of the two vertices $z$ and $y$, say $z$, is in $S$ then $V(M)=\{z, p\}$ and hence every vertex of $A$ which is not adjacent to at least one other vertex of $A$ is central. It follows from the minimality of the degree of $a$ as a central vertex that the degree of every vertex of $\bar{A}$, and in particular of $z$, is at most two. Hence if $z$ is to have degree less than two then $|V(A)| \leq 4$. From Case 1 we get that $|V(A)|-2 \geq|V(M)|>1$ and hence $|V(A)|=4$. We are now in the situation that $y$ is not adjacent to $a$ which is not adjacent to $z$ which is not adjacent to some other vertex and $\bar{A}$ is a forest. Hence $\bar{A}$, and therefore $A$, is equal to $P_{3}$. Then only $z$ has degree at most one in $J$ and hence $\alpha(w)=z$ which implies $A \cong J-z-y$. This is impossible because $a$ has in the graph $J-z-y$ degree three.

If there is exactly one vertex $x$ of $A$ which is not adjacent to $a$, then $V(M)=\{x, p\}$ and the vertices $x$ and $p$ are not adjacent. Hence every vertex of $A$ not adjacent to at least one other vertex of $A$ is central. If a vertex $y$ of $A$ is not adjacent to at least two vertices of $A$, then $y$ is central but has smaller degree than the vertex $a$. Thus every vertex of $A$ is not adjacent to at most one other vertex of $A$. This means that $\bar{A}$ is the sum of edges and singletons. If $\bar{A}$ is the sum of at least two edges, then every vertex of $A$ has at least degree two. If $\bar{A}$ is the sum of at least three paths, then every vertex of $A$ has at least degree two. Hence $|V(A)| \leq 3$. Because we can assume that $|V(A)| \geq|V(M)|+2$ this is not possible. Hence $J \in \operatorname{Forb}(H)$.

Observe that we have now established the lemma if $m=2$. We assume therefore from now on that $m>2$. Cases 3 and 4 will be used in the proof of Case 5, which establishes the lemma in the case that $m>2$.

Case 3: $\bar{M}$ is a triangle and $\bar{A}$ consists of a triangle together with two endpoints attached at different vertices of the triangle. Let $V(A)=\{a, b, c, d, e\}$ and assume that the vertices $a, b$ and $c$ are pairwise nonadjacent. Assume that $d$ is adjacent to $b$ and $c$ but not to $a$, while $e$ is adjacent to $c$ and $a$ but not to $b$. Let $q \notin V(A)$ and $J$ be a graph with vertex set $V(J)=V(A) \cup\{q\}$. The edges of $J$ are such that $J \mid V(A)=A$ and $x \in V(A)$ is adjacent to $q$ if and only if $x \in\{d, e\}$. Clearly $A$ can be embedded into $J$ and $J \mid\{a, b, c, q\}$ is isomorphic to $D \cong \overline{K_{4}}$. The vertex $u$ of $G$ is adjacent to four pairwise 
nonadjacent vertices while $J$ does not contain such a vertex. If $H$ could be embedded into $J, H$ and $J$ would have to be isomorphic, but $J$ does not contain an isolated vertex. Hence $J \in \operatorname{Forb}(G, H)$.

Case 4: Every copy of $C$ in $A$ contains the vertex $a$, every vertex of $A-M-a$ is in a copy of $C$, for every copy $\alpha(C)$ of $C$ in $A,|V(\alpha(C))-V(M)-\{a\}| \leq 1$ holds and the vertex $p$ is an isolated vertex of $M$. Because $p$ is an isolated vertex of $M$, there is an automorphism of $G$ which switches $v$ with $p$ and is the identity on every other vertex of $G$. Let $b$ be a vertex not in $V(A) \cup\{p\}$. Let $J$ be the graph with vertex set $V(J)=V(A) \cup\{b, p\}$. The edges of $J$ are such that $J|V(A)=A, J| V(M)=M$ and $a$ is adjacent to both vertices $b$ and $p$. No vertex of $M-p$ is adjacent to either $p$ or $b$. Each vertex of $A-(M-p)-a$ is adjacent to exactly one of the vertices $b$ and $p$ in such a way that the degrees of $b$ and $p$ are as equal as possible. The vertices $b$ and $p$ are not adjacent. Clearly, $A$ can be embedded into $J$ and $J \mid(V(M) \cup\{b\})$ is isomorphic to $D$. We will prove that $J \in \operatorname{Forb}(G, H)$.

Relying on Case 1, we will assume that $|V(A)| \geq|V(M)|+2$. Note that the graph $A$ consists of copies of $C$ which pairwise have exactly the vertex $a$ in common. If $\alpha(C)$ and $\beta(C)$ are two different copies of $C$ in $A$, then every vertex of $\alpha(C)-a$ is adjacent to every vertex of $\beta(C)-a$ and also either $V(\alpha(C)) \subseteq V(M-p) \cup\{a\}$ or $|V(\alpha(C))-V(M)-\{a\}|=1$ holds. Let $C_{0}, C_{1}, \ldots, C_{r-1}$ be the copies of $C$ in $A$ with, for all $i \in r, V\left(C_{i}\right) \subseteq V(M-$ $p) \cup\{a\}$ and let $D_{0}, D_{1}, \ldots, D_{s-1}$ be the other copies of $C$ in $A$. Let $d_{i}$ be the unique element in the set $V\left(D_{i}\right)-V(M-p)-\{a\}$. Observe that $V(M-p)=$ $\bigcup_{i \in r} V\left(C_{i}\right) \cup \bigcup_{i \in s} V\left(D_{i}-d_{i}\right)$ and $V(A)=V(M-p) \cup\{a\} \cup\left\{d_{i}: i \in s\right\}$. Because $|V(A)| \geq|V(M)|+2$, we get that $s \geq 2$. The vertices $V(C-p)$ are in at most one of the dual blocks of $A$. Otherwise $\overline{C-p}$ would be disconnected and hence $\bar{C}$ not two-connected, hence $\geq 1$. Because $m \geq 3$ we get $\left|V\left(D_{i}-d_{i}-a\right)\right| \geq 1$ for all $i \in s$. Those facts imply that $|V(M)| \geq m+2(m-2) \geq 2 m-1 \geq m+2$.

Assume for a contradiction that $\alpha$ is an embedding from $G$ into $J$. Observe first that it is not possible that $\alpha(v) \in V(A)-a$, because there is some copy $C^{\prime}$ of $C$ such that $\alpha(v) \in V\left(C^{\prime}-a\right)$. The only vertices of $J$ to which $\alpha(v)$ is possibly not adjacent are the vertices of $C^{\prime}-\alpha(v)$ and the vertices $p$ and $b$. But $\left|V\left(C^{\prime}-\alpha(v)\right) \cup\{p, b\}\right|=m+1$. On the other hand $\alpha(v)$ is not adjacent to every vertex in $\alpha(M)$ and $|V(M)| \geq m+2$. Because $v$ and $p$ are exchangeable, we also get that $\alpha(p) \notin V(A)-a$. But this means that neither $\alpha(p)=a$ nor $\alpha(v)=a$. For assume without loss of generality that $\alpha(v)=a$. Then $\alpha(p) \in V(J-a)$ and hence because $\alpha(p) \notin V(A)-a$, we get using the previous argument that $\alpha(p) \in\{p, b\}$. This is not possible since $p$ and $v$ are not adjacent to each other but both vertices $p$ and $b$ are adjacent to $a$. Hence, $\{\alpha(p), \alpha(v)\}=\{p, b\}$. Both of the vertices $p$ and $v$ are not adjacent to any vertex in $C-p$ and thus $V(\alpha(C-p)) \subseteq V(M-p)$. The vertex $u$ is adjacent to both vertices $p$ and $v$, and $a$ is the only vertex of $J$ adjacent to both vertices $p$ and $b$. Hence $\alpha(u)=a$. But this is a contradiction because the vertex $\alpha(u)=a$ is not adjacent to any vertex of $V(\alpha(C-p)) \subseteq V(M-p)$.

Assume next that there is an embedding $\alpha$ from $H$ into $J$. The vertex $w$ is an isolated vertex of $H$ and hence $\alpha(w)$ must be a vertex of degree one of $J$. The vertex $a$ has degree two. The graph $A$ has at least two dual blocks, otherwise $|V(A)|=|V(M)|$. Also, because $m>2$, every dual block of $A$ 
contains at least three vertices. Hence, every vertex of $A-a$ has degree at least two. The only vertices of $J$ which might have degree one are therefore $b$ and $p$. Assume without loss of generality that the degree of $b$ is less than or equal to the degree of $p$. The degree of $b$ is one only if $|V(A)|=|V(M)|+1$. This situation has been dealt with in Case 1 .

Case 5: $m \geq 3$. Because $A$ is an $M$-tree, it follows that the hypergraph induced by the copies of $C$ in $A$ does not contain a circuit. Hence, there exists a colouring $\Delta: V(A) \rightarrow\{$ red, blue $\}$ such that every vertex in $V(M-p) \cup\{a\}$ is red, every copy of $C$ in $A$ contains at least as many red vertices as blue vertices and if $\Delta$ is constant on the vertices of a copy of $C$ in $A$, then this copy is an induced subgraph of $A \mid(V(M-p) \cup\{a\})$. Any such colouring $\Delta$ of the vertices of $A$ will be called a good colouring. Of course, such a good colouring $\Delta$ of $A$ need not be unique because not every element of $A$ is necessarily in a copy of $C$, the hypergraph induced by the copies of $C$ in $A$ need not be connected and the colouring of the vertices of a single copy of $C$ need not be unique. For each such colouring $\Delta$ there is a graph $J=J(\Delta)$ which we will construct as follows. First choose a new vertex $b \notin V(A) \cup\{p\}$.

The graph $J$ has the vertex set $V(J)=V(A) \cup V(M) \cup\{b\}=V(A) \cup\{p\} \cup\{b\}$. The edges of $J$ are such that $J|V(A)=A, J| V(M)=M$. The vertex $x \in$ $V(J-M)$ is adjacent to $p$ if and only if $x \neq b$ and $\Delta(x)=$ red. The vertex $x \in V(J)$ is adjacent to $b$ if and only if $\Delta(x)=$ blue or $x=a$. It follows that the vertex $a$ is adjacent to both vertices $p$ and $b$. Note that every copy $C_{0}$ of $C$ in $A$ contains at least one vertex adjacent to $p$ and at least one vertex adjacent to $b$. If $a$ is a vertex of $C_{0}$ this is clearly the case. If $a$ is not a vertex of $C_{0}$, then $C_{0}$ contains at most one vertex which is also a vertex of $M$. Otherwise, the hypergraph given by $\mathfrak{S}$, the dual blocks of $A$, would contain a circuit. We conclude that $\left|V\left(C_{0}\right)-V(M)\right| \geq 2$. By the definition of $\Delta$ there is a red vertex in $V\left(C_{0}\right)-V(M)$ which is adjacent to $p$ and there is a blue vertex in $V\left(C_{0}\right) \cap V(A-(M-p)-a)$ which is adjacent to $b$. Clearly there is an embedding from $A$ into $J$ and $J \mid(V(M) \cup\{b\})$ is isomorphic to $D$.

We wish to argue that if $|V(A)| \geq|V(M)|+2$ and all the conditions of Case 4 are not satisfied, then there is a good colouring $\Delta$ of $A$ such that in $J=J(\Delta)$ both of the vertices $p$ and $b$ have degree at least two. We will therefore investigate in order each of the conditions posed in Case 4 and show for each such condition that if it is violated then there is a good colouring $\Delta$ such that both vertices $p$ and $b$ have degree at least two in $J(\Delta)$. Remember that both vertices $p$ and $b$ are adjacent to the vertex $a$.

Assume that there is a copy $\alpha(C)$ of $C$ in $A$ such that the vertex $a \notin$ $V(\alpha(C))$. Then $|V(\alpha(C)) \cap(V(M) \cup\{a\})| \leq 1$ because the dual blocks of $A$ do not form a circuit and the hypergraph of dual blocks of $A$ restricted to $V(M-p) \cup\{a\}$ is a star with center $a$. If $\Delta$ is then any good colouring of $A$, at least one vertex of $V(\alpha(C))-(V(M) \cup\{a\})$ will be coloured red and some other vertex blue. This means that in $J=J(\Delta)$ both vertices $p$ and $b$ have degree at least two.

Assume that there is a vertex $x \in V(A-M-a)$ which is not in any copy of $C$ in $A$. Let $y$ be some other vertex in $V(A-M-a)$ and $\Delta$ a good colouring of $A$. (There must be such a vertex $y \neq x$ otherwise $|V(A)| \leq|V(M)|+1$.) If $\Delta(x) \neq \Delta(y)$, then both vertices $p$ and $b$ have degree at least two in $J(\Delta)$. If $\Delta(x)=\Delta(y)=$ red change $\Delta$ to $\Delta^{\prime}$ by changing the colour of the vertex $x$ from 
red to blue. Observe that $\Delta^{\prime}$ is again a good colouring. If $\Delta(x)=\Delta(y)=$ blue change $\Delta$ to $\Delta^{\prime}$ by changing the colour of the vertex $x$ from blue to red. Observe that $\Delta^{\prime}$ is again a good colouring.

If there is a copy $\alpha(C)$ of $C$ in $A$ such that for $N=V(\alpha(C))-V(M)-\{a\}$, $|N| \geq 2$ holds. We may also assume that every copy of $C$ in $A$ contains the vertex $a$ because if that last condition is violated we have already shown that then there is a colouring $\Delta$ such that the vertices $p$ and $q$ have at least degree two in $J(\Delta)$. Let $\Delta$ be a good colouring of $A$. Observe that if not all of the vertices of $N$ receive the same colour then the degree of $p$ and $b$ in $J(\Delta)$ is at least two. If all of the vertices in $N$ are coloured red, then choose a vertex $x$ in $N$ and change the colouring $\Delta$ to the colouring $\Delta^{\prime}$ by changing the colour of the vertex $x$ from red to blue. Observe that $\Delta^{\prime}$ is a good colouring of $A$. If all of the vertices in $N$ are coloured blue, then choose a vertex $x$ in $N$ and change the colouring $\Delta$ to the colouring $\Delta^{\prime}$ by changing the colour of the vertex $x$ from blue to red. Observe that $\Delta^{\prime}$ is a good colouring of $A$.

If $p$ is not an isolated vertex of $M$, then the vertex $p$ has degree at least two in $J$ because $p$ is also adjacent to the vertex $a$. To see that the vertex $b$ has degree at least two in $J$ we have to prove that it is not possible that the colouring $\Delta$ which colours every vertex of $A$ with the colour red is the unique good colouring of $A$. If this where the case, choose a vertex $y$ in $V(A-M-a)$ and change the colouring $\Delta$ to the colouring $\Delta^{\prime}$ by changing the colour of the vertex $y$ from red to blue. It is easy to see that $\Delta^{\prime}$ is a good colouring of $A$ and that in $J\left(\Delta^{\prime}\right)$ both of the vertices $p$ and $b$ have a degree of at least two.

We conclude that we may assume that the vertices $p$ and $b$ of the graph $J=J(\Delta)$ have degree at least two.

We will prove that $J \in \operatorname{Forb}(G)$. Assume to the contrary that $\alpha$ is an embedding from $G$ into $J$. We argue first that it is not possible that both $\alpha(v) \in V(A)$ and also $\alpha(M)$ contains at most one of the two vertices $p$ and $b$. Note that $\alpha(M)$ then contains exactly one of the two vertices $p$ and $b$ because $D$ cannot be embedded into $A$. Let $r \in\{p, b\} \cap V(\alpha(M))$. No vertex of $\alpha(M)$ is adjacent to the vertex $\alpha(v)$, hence in particular no vertex of $\alpha(M)-r$ is adjacent to $\alpha(v)$. This means that $\alpha(v)$ is a central vertex of $A$ and hence according to Proposition $1, r$ is not adjacent to any of the vertices of $\alpha(C)-r$ and $r$ is not adjacent to the vertex $\alpha(v)$, because $r \in V(\alpha(M))$. According to Proposition 1, $A \mid(V(\alpha(C)-r \cup \alpha(v))$ is an isomorphic copy of $C$. But this is not possible because the vertex $r \in\{p, b\}$ and every copy of $C$ in $A$ contains one vertex adjacent to $p$ and one vertex adjacent to $b$.

Next we investigate the case $\alpha(u)=a$. Then $V(\alpha(D)) \subseteq V(J-(M-p)-a)$. If $\alpha(v)=p$ then $b \in V(\alpha(C))$ because every copy of $C$ in $A$ contains a vertex adjacent to $p$. Every vertex $x$ of $\alpha(C)-b$ is in $V(A-(M-p)-a)$ and is not adjacent to $p$. Hence $x$ is adjacent to $b$. The graph $C$ then contains a vertex adjacent to every other vertex of $C$ in contradiction to the fact that $C$ is a dual block of $A$. Similarly $\alpha(v) \neq b$. If $\alpha(v) \in V(A-(M-p)-a)$ then $\alpha(v)$ is adjacent to $p$ or to $b$ and hence $\alpha(M)$ contains at most one of the two vertices $p$ and $b$ which is not possible according to the previous paragraph. The vertex $\alpha(v) \notin V(M-p)$ because $\alpha(v)$ is adjacent to $\alpha(u)=a$. We conclude that $\alpha(u) \neq a$.

If $\alpha(u)=b$, then $\alpha$ would embed $D$ into $A$ because $b$ and $p$ are not 
adjacent. Similarly $\alpha(u) \neq p$. Hence $\alpha(u) \in V(A-a)$ and because no vertex of $A-a$ is adjacent to both vertices $b$ and $p, V(\alpha(D))$ contains exactly one of the two vertices $b$ or $p$. Also, $\alpha(v) \neq b$ because then $\alpha$ would embed $M$ and hence $C$ into $A$ and every copy of $C$ in $A$ contains at least one vertex adjacent to $b$. Similarly $\alpha(v) \neq p$. Hence $\alpha(v) \in V(A)$ and $\alpha(M)$ contains exactly one of the two vertices $b$ and $p$ which, as we saw above, is impossible. Hence $J \in \operatorname{Forb}(G)$.

We prove next that $J \in \operatorname{Forb}(H)$. Assume to the contrary that $\alpha$ is an embedding from $H$ into $J$. The number of vertices of $J$ is exactly one more than the number of vertices of $H$. Hence, if there is an embedding from $H$ into $J$ then there exists a vertex $x \in V(J)$ such that $H$ is isomorphic to $J-x$. The graph $H$ contains the isolated vertex $w$ which means that $J$ contains the vertex $\alpha(w)$ with degree at most one. Using Case 1, we can assume that $|V(A)| \geq|V(M)|+2$. Both vertices $p$ and $b$ have degree at least two. Hence $\alpha(w) \in V(A)$. Let $F=V(C-p) \cup\{a\}$. According to Proposition $1, F$ is a copy of $C$ in $A$ and no vertex of $C-p=F-a$ is adjacent to $p$. Assume that $y \in V(A)$ is a vertex which has degree at most one in $A$. If $y \notin V(F)$, then because $|V(F)| \geq 3$ and the degree of $y$ is at most one, $\bar{A} \mid(V(F) \cup\{y\})$ is two-connected. But this is not possible because $A$ is an $M$-tree and $|V(F) \cup\{y\}| \geq m+1$. Hence $y \in V(F)$. We conclude that $\alpha(w) \in V(F)$.

The vertex $\alpha(w) \neq a$ because the vertex $a$ has degree at least two in $J$ and hence $\alpha(w) \in V(C-p)$. If $\alpha(w)$ is a vertex of degree zero in $A$, then because $\alpha$ embeds $A$ into $J-\alpha(w)$ and $|V(A)|=|V(J-\alpha(w))|-1, J-\alpha(w)$ contains a vertex $y=\alpha(\alpha(w))$ of degree at most one. Both vertices $p$ and $b$ are not adjacent to the vertex $\alpha(w) \in V(C-p)$, hence have degree at least two in $J-\alpha(w)$, and hence $y \in V(A)$. Of course then $y \in F$ because $y$ has degree at most one in $A$ and is therefore not adjacent with at least two vertices of $F$ putting it into the same dual block as the vertices of $F$. The vertices $y$ and $\alpha(w)$ are together in only one dual block of $A$ and this block is $F$. Every vertex of $A$ is in some common dual block with $\alpha(w)$. Every vertex of $A$ except possibly one is in some common dual block with $y$. The only dual block of $A$ containing $y$ which has a point in common with a dual block containing $\alpha(w)$ is $F$. Hence $|V(A)| \leq|V(F)|+1 \leq m+1 \leq|V(M)|+1$. We conclude that $\alpha(w)$ has degree one in $A$.

Assume that $\alpha(w)$ is only adjacent to the vertex $x \in V(A)$ and $\alpha(w) \in$ $C-p$. If $x \in V(F)$, every dual block of $A$ contains $\alpha(w)$. This means that $F$ is the only dual block of $A$ containing $a$ and hence $F-a \cong M-p$. Because $p$ is an isolated vertex of $C$ we get that $F \cong M$ holds. Hence $\alpha(w)$ is a central vertex, for $M-x$ instead of $M-p$, of $A$. Because $|V(A)|>|V(M)|$ there is at least one other dual block containing $\alpha(w)$ and every vertex in it is adjacent to $a$, in contradiction to the assumption that $a$ has minimal degree amongst all central vertices of $A$. Hence, $x \notin V(F)$.

If $x \notin V(D)$ there is at most one dual block which does not contain $\alpha(w)$ and the only vertices it contains are $x$ and possibly also $a$. All the other dual blocks of $A$ contain $\alpha(w)$. If $M-p=J \mid(V(F-a) \cup\{x\})$, then $a$ is not adjacent to $x$ and because $|V(A)|>|V(M)|+1$, there are at least two vertices in dual blocks of $A$ containing $\alpha(w)$ and adjacent to $a$. Hence $\alpha(w)$ 
would be a central vertex of smaller degree than $a$. This implies that $M \cong F$ and hence $\alpha(w)$ is a central vertex. Because $|V(A)|>|V(M)|+1$ there is at least one vertex in a dual block of $A$ containing $\alpha(w)$ and adjacent to $a$. Hence $x$ is not adjacent to $a$ by the minimality of the degree of $a$ as a central vertex. The vertices of $F-a$ are not adjacent to $b$ and not adjacent to $p$. Also, $V(F-\alpha(w)) \cup\{p, b\} \subseteq V(\alpha(A))$, because the vertices $\alpha(w)$ and $x$ are not in $V(A)$ and $|V(A)|=|V(J-\alpha(w)-x)|$. If $m \geq 4$, the complement of the graph $J \mid(V(F-\alpha(w)) \cup\{p, b\})$ is two-connected and contains $m+1$ vertices which is impossible because $A$ is an $M$-tree. It follows that $m=3$. Hence $\bar{M}$ is a triangle and $\bar{A}$ consists of a triangle together with two endpoints attached at different vertices of the triangle. (This is so because every vertex of $A-F-x$ is adjacent to $a$ but not to $\alpha(w)$. Hence, because of the minimality of the degree of $a$ as a central vertex, there is at most one such vertex.) This implies that we arrived at a situation dealt with by Case 3 . We conclude that $J \in \operatorname{Forb}(G, H)$.

\subsection{The SECOND MAIN LEMMA FOR CASE 2}

Lemma 13. Let $A$ and $M$ with $|V(A)|>|V(M)|>1$ be graphs such that there is an embedding $\gamma$ from $M$ into $A$. For $u, v$, and $w$, three vertices not in $A$ or $M$, the graphs $D, H$, and $G$ are given by $D=v+M, G=u \oplus D$, and $H=w+A$. The graphs $A$ and $M$ are elements of $\operatorname{Forb}(G, H)$ and $A$ is an $M$ tree. Then either $M$ is complete, or there exists a graph $J \in \operatorname{Forb}(G, H)$ such that both graphs $A$ and $D$ can be embedded into $J$, or $G \cong K_{1} \oplus\left(K_{1}+K_{1}+K_{1}\right)$ and $H \cong K_{1}+P_{2}$.

Proof. If $M$ contains an isolated vertex $p$, then for $a=\gamma(p)$ and for $\delta=$ $\gamma \mid(M-p)$ we can apply Lemma 12 . Hence we will assume that $M$ does not contain an isolated vertex. If $|V(M)|=2$ and $M$ is not complete then $M \cong K_{2}$ and hence $M$ contains an isolated vertex. We assume therefore from now on that $|V(M)| \geq 3$. We can assume that there is no embedding from $A$ into $D$ because then we could choose $J=D$ and that there is no embedding from $D$ into $A$ because we could then choose $J=A$. Let $\mathfrak{S}$ denote the set of dual blocks of $A$. That is $S \in \mathfrak{S}$ if and only if $S \subseteq V(A), \overline{J \mid S}$ is two-connected and if $S \subset T \subseteq A$ then $\overline{J \mid T}$ is not two-connected. Those dual blocks form a hypergraph on $A$ which does not contain a circuit. In particular any two elements of $\mathfrak{S}$ have at most one vertex in common.

We will first discuss three special cases for the graphs $A$ and $M$ and then a colouring of hypergraphs which we will use to colour the vertices of the hypergraph of dual blocks $\mathfrak{S}$ of $A$. Certain properties of this colouring will be utilised in the main part of the proof of Lemma 13.

Case 1: Every vertex of $M$ has degree one if the number $|V(M)|$ is even and at least four if the set $V(M) \subseteq V(A)$, and if the set $S \in \mathfrak{S}$ has the property that $|S|=|V(M)|$ then $S=V(M)$. Then $M$ consists of a single dual block. Let $a \in V(A)-V(M)$. If $a$ is not adjacent to two different vertices of $M$ then $\overline{J \mid(V(M) \cup\{a\})}$ is two-connected and hence $V(M) \cup\{a\}$ is a dual block of $A$. This is a contradiction to the fact that $A$ is an $M$-tree. If there is a vertex in $V(A)-V(M)$ which is not adjacent to $a$ we denote this vertex by $y$. The vertex $a$ is adjacent to all vertices of $M-y$. Hence there are two adjacent vertices $c, d \in V(M)$ such that both vertices $c$ and $d$ are adjacent 
to $a$. Let $r, b$ be two different vertices with $r, b \notin V(A)$. We let $J$ be the graph with vertex set $V(J)=V(A) \cup\{r, b\}$. The edges of $J$ are such that $J \mid V(A)=A$, the vertex $x \in V(A)$ is adjacent to $r$ if and only if $x=c$, the vertex $x \in V(A)$ is adjacent to $b$ if and only if $x=d$ and the vertices $r, b$ are not adjacent. Clearly $A$ can be embedded into $J$ and $D$ is isomorphic to $J \mid(V(M-c) \cup\{r, b\})$.

We prove first that $J \in \operatorname{Forb}(G)$. Assume to the contrary that $\alpha$ is an embedding from $G$ into $J$. The vertex $\alpha(u) \neq r$ and the vertex $\alpha(u) \neq b$ because $u$ has degree at least five and both vertices $r$ and $b$ have degree one. If $\alpha(u) \in V(A)-\{c, d\}$ then $V(\alpha(D)) \subseteq V(A)$ because $\alpha(u)$ would then not be adjacent to either $r$ or $b$. We assume therefore without loss of generality that $\alpha(u)=c$. Every vertex of $V(\alpha(M))$ is adjacent to $c$ and hence $V(\alpha(M)) \subseteq V(A-c) \cup\{r\}$. The vertex $r \notin V(\alpha(M))$ because $r$ has degree zero in $J-c$ and $M$ does not contain an isolated vertex. The only vertex of $M$ adjacent to $c$ is $d$ and hence $V(\alpha(M)) \cap V(M) \subseteq\{d\}$. Then for some $S \in \mathfrak{S}, V(\alpha(M))=S$ with $S \neq V(M)$, a contradiction. Hence $J \in \operatorname{Forb}(G)$.

We prove next that $J \in \operatorname{Forb}(H)$. Assume to the contrary that $\alpha$ is an embedding from $H$ into $J$. In the graph $H$ the vertex $w$ is an isolated vertex, not adjacent to any vertex of $A$ and $|V(J)|=|V(H)|+1$, which implies that the degree of $\alpha(w)$ in $J$ is at most one. If $\alpha(w)=r$, then $V(\alpha(A))=$ $V(A-c) \cup\{b\}$. The vertex $c$ is adjacent to $d$ and the vertex $a$ in $A$. The vertex $b$ is adjacent in $V(\alpha(A))=V(A-c) \cup\{b\}$ only to $d$. Hence $A$ would contain more edges than $V(\alpha(A))=V(A-c) \cup\{b\}$. We conclude similarly that $\alpha(w) \neq b$. To every vertex $z \in V(A)-V(M)$ there is at most one vertex in $V(M)$ which is not adjacent to $z$. Hence every vertex in $V(A)-V(M)$ has at least degree three. If the vertex $a$ is not adjacent to the vertex $y \in V(M)$, the degree of every vertex in $V(M-y)$ is at least two. This leaves $\alpha(w)=y$. Denote by $z$ the vertex which is adjacent to $y$ in $M$. Then $V(\alpha(A))=$ $V(A-y-z) \cup\{r, b\}$. This is not possible because the only possible vertex of $A$ which has degree one is $y$, while in $V(\alpha(A))=V(A-y-z) \cup\{r, b\}$ both vertices $r$ and $b$ have degree one.

Case 2: The graph $M$ contains a vertex $z$ which is not adjacent to all vertices in $M$. The graph $A$ contains the graph $M$ as an induced subgraph and every vertex $x \in V(A)-V(M)$ is adjacent to all vertices in $V(A-z)$ but not to the vertex $z$. Because $|V(A)|>|V(M)|$ we can choose a vertex $y \in V(A)-V(M)$. Let $b$ be a vertex such that $b \notin V(A)$. The graph $J$ with vertex set $V(J)=$ $V(A) \cup\{b\}$ is defined to be such that $J \mid V(A)=A$ and that the set of vertices in $J$ adjacent to $b$ is the set $V(A)-V(M)$. The identity map is an embedding from $A$ into $J$ and $J \mid(V(M) \cup\{b\})$ is an embedding from $D$ into $J$. In order to finish the proof of Case 2 it will be sufficient to show that $J \in \operatorname{Forb}(G, H)$.

Assume for a contradiction that there is an embedding $\alpha$ from $G$ into $J$. The vertex $b$ is only adjacent to the vertices in the set $V(A)-V(M)$ and hence if $\alpha(u) \notin V(A)-V(M)$ then $V(\alpha(D)) \subseteq V(A)$ and $D$ could be embedded into $A$. Hence $\alpha(u) \in V(A)-V(M)$. If $\alpha(v)=b$ then $\alpha(M)=M$ because all vertices in $V(A)-V(M)$ are adjacent to the vertex $b$. It is not possible that $\alpha(M)=M$ and $\alpha(u) \in V(A)-V(M)$ because no vertex in $V(A)-$ $V(M)$ is adjacent to the vertex $z$. If $\alpha(v) \in V(A)-V(M)$ then $V(M)=$ $\{z\}$ in contradiction to $|V(M)|>1$. Hence $\alpha(v) \in V(M)-\{z\}$. Every vertex of $\alpha(M)$ is adjacent to $\alpha(u)$ and not adjacent to $\alpha(v)$. This implies 
that $V(\alpha(M)) \subseteq V(M-z-\alpha(v)) \cup\{b\}$ which is a contradiction because $|V(M-z-\alpha(v)) \cup\{b\}|<|V(M)|$.

Next we will assume for a contradiction that $\alpha$ is an embedding from $H$ into $J$. Note that $|V(H)|=|V(J)|$ and hence $J \cong H$ which implies that $J$ has an isolated vertex because $H$ contains the isolated vertex $w$. The set $V(A)-V(M) \neq \varnothing$ because otherwise $|V(A)|=|V(M)|$. Every vertex $x \in$ $V(A)-V(M)$ is adjacent to $b$ and every vertex of $A$ except $z$ to the vertices in $V(A)-V(M)$. This implies that $\alpha(w)=z$ which is in contradiction to our general assumption that the graph $M$ does not have an isolated vertex.

The graph $M$ is called a tussel if $\mathfrak{S}$ contains an element $S$ with at least three vertices and every other element $T$ of $\mathfrak{S}$ contains exactly two vertices and $T \cap S \neq \varnothing$. If $M$ is a tussel we call the largest dual block the centerblock of the tussel and will usually denote the subgraph of $M$ induced by the centerblock by $C$. If $M$ is a tussel then for every $x \in V(M)-V(C)$ there is a vertex $f(x) \in V(M)$ which is not adjacent to $x$ and for all vertices $x \in V(M)-V(C)$, $f(x) \in V(C)$.

Case 3: The graph $\bar{M}$ is a tussel and the subgraph $C$ induced by the centerblock does not contain any edges. Let $f(x)$ be the function which associates with every vertex $x \in V(M)-V(C)$ the unique vertex not adjacent to $x$. Note that the function $f$ is not constants for otherwise $M$ would have an isolated vertex. This implies in particular that $V(M)-V(C)$ contains at least two elements. We will assume that the embedding $\gamma$ from $M$ into $A$ is the identity map. Choose a vertex $c \in V(C)$ such that it has maximal degree in $M$ for all vertices of $C$. Denote the set of vertices of $M$ which is adjacent to $c$ by $N$. It follows from the fact that $f$ is not constant and from the choice of $c$ that there are at least two vertices $x \in V(M)-V(C)$ with $f(x) \neq c$ and hence with $x$ and $c$ not being adjacent. Hence $|N| \geq 2$. Let $X$ be the set of all vertices $x \in V(A)$ such that there is a set $S \in \mathfrak{S}$ with $x \in S$ and $|S \cap V(C-c)|=1$. Note that $N \subseteq X$ and hence $|X| \geq 2$.

Because no dual block of $M$ contains an edge and $A$ is an $M$-tree it follows that the subgraphs of $A$ induced by the elements of $\mathfrak{S}$ do not contain an edge. Let $\Lambda$ be a colouring of the set of vertices of $A$ with two colours, say red and blue, such that $\Lambda(c)=$ blue, all vertices of $V(M-c)$ are red, every set $S \in \mathfrak{S}$ contains at most one blue vertex and if $\Lambda$ is constant to red on some $S \in \mathfrak{S}$ then $S \subseteq V(M)$. Such a colouring exists because the hypergraph of dual blocks of $A$ does not contain a circuit. If $S \in \mathfrak{S}$ and $S \subseteq V(M)$ then either $S=V(C)$ and hence contains a blue vertex or $|S|=2$. Hence every $S \in \mathfrak{S}$ with $|S| \geq 3$ contains a blue vertex and in particular every $S \in \mathfrak{S}$ with $|S|=|V(C)|$ contains a blue vertex.

Choose two vertices $r$ and $b$ with $\{r, b\} \cap V(A)=\varnothing$. The graph $J$ is defined to have vertex set $V(A) \cup\{r, b\}$ and the edges of $J$ are such that $J \mid V(A)=A$, the vertices $r$ and $b$ are not adjacent and a vertex $x \in V(A)$ is adjacent to $b$ if and only if it is blue. The vertex $r$ is adjacent to a vertex $x$ in $A-C$ if and only if $x$ is red or $x \in X$. No vertex in $V(C)$ is adjacent to $r$. Note that the vertex $r$ is adjacent to a vertex $x \in V(M)$ if and only if $x \in N$. Hence $r$ is adjacent to a vertex $x$ in $M$ if and only if $x$ is adjacent to $c$. The blue vertices of $X$ are the only vertices of $V(J)$ adjacent to both vertices 
$r$ and $b$. The identity map embeds $A$ into $J$ and $J \mid(V(M-c) \cup\{r, b\})$ is isomorphic to the graph $D$.

Note that every $S \in \mathfrak{S}$ with $|S|=|V(M)|$ contains a vertex which is adjacent to $b$. Let $\alpha$ be an embedding from $M$ into $A$. We will show that $V(\alpha(M))$ contains a vertex which is adjacent to $r$. All but one vertex of $\alpha(C)$ are red and $|V(\alpha(C))| \geq 3$ and hence $\alpha(C)$ contains a vertex adjacent to $r$ if $\alpha(C) \neq C$. If $\alpha(C)=C$ then for a vertex $x \in V(M)-V(C)$ which is adjacent to $c$ the vertex $\alpha(x) \in S$ for some $S \in \mathfrak{S}$ with $|S \cap V(C)-c|=1$ and hence $\alpha(x)$ is adjacent to $r$.

In order to complete the proof for Case 3 it remains to show that $J \in$ $\operatorname{Forb}(G, H)$. Assume for a contradiction that there is an embedding $\alpha$ from $G$ into $J$. First we investigate the value of $\alpha$ at $u$. The vertex $u$ is adjacent to every other vertex of $G$. If $\alpha(u)=b$ then because $r$ and $b$ are not adjacent the map $\alpha$ would embed $D$ into $A$. If $\alpha(u)=r$ then because $r$ and $b$ are not adjacent the map $\alpha$ would embed $D$ into $A$. Hence $\alpha(u) \in V(A)$.

Then we investigate the value of $\alpha$ at $v$. The vertex $v$ is not adjacent to any vertex in $M$. Hence $\{v\} \in V(C)$ is a set of independent vertices. Let $T=\{v\} \cup V(C)$. Because $A$ is an $M$-tree it follows that $\alpha(T) \nsubseteq V(A)$ and hence at least one of $r$ and $b$ in $\alpha(T)$. The set $\alpha(T) \cap V(A)$ contains at least $|V(C)|-1$ vertices and hence there is a unique $S \in \mathfrak{S}$ with $\alpha(T) \cap V(A) \subseteq S$. If $S \neq V(C)$ then $r \notin \alpha(T)$ because then $r$ is adjacent with all but one vertex of $S$ and hence with a vertex in $\alpha(T) \cap V(A)$. (If $S$ has nonempty intersection with $V(C-c)$ then $r$ is adjacent with all vertices of $S$ which are not in $V(C)$. If $S$ does not have any vertices in $V(C)$ then $r$ is adjacent with all vertices of $S$ except the blue vertex. If $S \cap V(C)=\{c\}$ then because not both vertices $b$ and $c$ are in $\alpha(T)$ there is a red vertex in $(\alpha(T) \cap V(A))-c$ which is adjacent to $b$.) If $r \notin \alpha(T)$ it follows that $\alpha(T) \cap V(A)=S$ and contains therefore a vertex adjacent to $b$. We conclude that $S=V(C)$.

If both vertices $r$ and $b$ are elements of $\alpha(M)$ then $\alpha(u) \in X$ and hence $a(u)$ is not adjacent to some vertex $y \in V(C-c)$. This implies that $\alpha(T)=$ $(V(C-y) \cup\{r, b\})$ which is impossible because the vertices $b$ and $c$ are adjacent. If $b$ is an element of $\alpha(T)$ then $\alpha(T)=V(C) \cup\{r\}$ which again is impossible because $b$ and $c$ are adjacent. Hence $r \in \alpha(T)$ and $b \notin \alpha(T)$. If $\alpha(v)=r$ then $V(\alpha(M)) \subseteq V(A)$ which is impossible because the vertex $r$ is adjacent to some vertex in every copy of $M$ in $A$. Hence $\alpha(v) \in V(A)$. We can then apply Lemma 12 with $\alpha(v)=a, \delta=\alpha$ and $p=\alpha^{-1}(r)$.

Hence $J \in \operatorname{Forb}(G)$. We prove next that $J \in \operatorname{Forb}(H)$.

Assume for a contradiction that there is an embedding $\alpha$ from $H$ into $J$. We will investigate the image of the vertex $w$ under the mapping $\alpha$. The vertex $w$ is isolated in $H$ and the graph $J$ has exactly one more vertex as does the graph $H$. Hence $\alpha(w)$ has in $J$ either degree zero or degree one. Because $X$ contains at least two elements the degree of $r$ and the degree of $b$ is at least two. Because $V(C)$ contains at least three elements every vertex in $V(A-C)$ is adjacent to at least two vertices in $C$. The vertex $c$ is adjacent to $b$ and to the vertices in $X$. This implies that $\alpha(w) \in V(C-c)$. The vertex $\alpha(w)$ is adjacent to at least one of the vertices in $X$. Say $\alpha(w)$ is adjacent to the vertex $d \in X$. Hence we arrive at the situation that every element of $\mathfrak{S}$ except one contains $\alpha(w)$ and the one element of $\mathfrak{S}$ which does not contain $\alpha(w)$ contains the vertex $d$ and exactly one other vertex, say $e \in V(C)$. The vertex 
$d \in X$ is the only vertex adjacent to $\alpha(w)$ and it has degree $|V(A)|-1$. The vertex $e$ is not adjacent to the vertex $d$ and has degree $|V(A)|-|V(C)|$. Each element of $\mathfrak{S}-V(C)$ contains exactly one vertex of $X$.

It follows that $A \cong J-\alpha(w)-d=\alpha(A)$ and that therefore the graph $J-\alpha(w)-d$ contains a vertex of degree one. If $X$ would contain at least three vertices then every vertex in $\alpha(A)$ would have degree at least two. Hence $X$ consists of exactly two vertices, the vertex $d$ and one other vertex, say $x$. Let $S$ be the element in $\mathfrak{S}$ such that $x \in S$; then $\mathfrak{S}=\{S, V(C),\{d, e\}\}$. The vertex $d \in V(A)$ is not adjacent to exactly one other vertex, the vertex $e$, of $A$. The only candidate for such a vertex in $J-\alpha(w)-d=\alpha(A)$ is the vertex $x$ and if this is the case the set $S$ contains exactly three vertices. Let $y \in S$ with $S=\{\alpha(w), x, y\}$. It follows that $a(d)=x$ and that $\alpha(e)=y$. This is impossible because the vertex $e$ has degree two in $A$ and the vertex $y$ is adjacent to $r$ and to at least two vertices in $V(C-\alpha(w))$.

Colouring. If $M$ is a tussel we choose a vertex $c$ in the centerblock $C$ of $M$ such that $c$ has maximal degree in $C$ amongst all vertices of $C$ and then if there are several choices we choose amongst all those vertices a vertex which has largest degree within $M$. If $M$ is not a tussel we choose the vertex $c$ as follows. If $C$ is a dual block of $M$ with a maximal number of vertices and $c \in V(C)$ a vertex such that $c$ has maximal degree as a vertex of $M$ amongst all of the vertices of $C$, we denote the degree of $c$ in $M$ by $v(C)$. We choose the maximal dual block $C$ of $M$ so that $v(C)$ is also maximal, and $c \in V(C)$ such that the degree of $c$ is $v(C)$. We denote by $N$ the set of all vertices in $M$ adjacent to the vertex $c$. Note that $|V(C)|>1$ because otherwise the graph $M$ would be complete. Let $M_{C}$ be the dual connected component of $M$ which contains $C .\left(\overline{M_{C}}\right.$ is connected, contains the vertices of $C$ and is maximal under these conditions.) Let $W$ be a graph which is isomorphic to the graph $M-M_{C}$ and let $W_{1}$ be the largest dual connected component of $A$ which can be embedded into $W$. We will assume that $V\left(W_{1}\right) \subseteq V(W)$ and that $\left(V(W)-V\left(W_{1}\right)\right) \cap V(A)=\varnothing$. Let $A_{1}$ be the graph with vertex set $V(A) \cup V(W)$ and the edges of $A_{1}$ are such that $A_{1}\left|V(A)=A, A_{1}\right| V(W)=W$ and $\bar{W}$ is a connected component of $\bar{A}_{1}$. That is, every vertex of $W$ is adjacent with every vertex in $A_{1}-W$. Let $B$ be the graph $A_{1}-W$. Note that then $A=B \oplus W$ and that $A=B \oplus W_{1}$. In order to simplify notation we will assume that the embedding $\gamma$ from $M$ into $A$ is the identity map on $M_{C}$ and that $M-M_{C}=W$. Then $V(M)=V\left(M_{C}\right) \cup V(W)$.

Note that $A_{1}$ is an $M$-tree. We will denote from now on by $\mathfrak{S}$ the set of dual blocks of $A_{1}$.

Let $\Lambda:\left(A_{1}\right) \rightarrow$ red, blue $\}$ be a colouring of the vertices of $V\left(A_{1}\right)$ with the colours red and blue such that the following colouring properties hold.

(i) The vertex $c$ is coloured blue.

(ii) All vertices of the graph $M-c$ are coloured red.

(iii) If the colouring $\Lambda$ is constant on an element $S \in \mathfrak{S}$ then all of the vertices of $S$ are coloured red and $S \subseteq V\left(M_{C}\right)$.

Such a colouring $\Lambda$ exists because the hypergraph with edge set $\mathfrak{S}$ does not contain a circuit. (There is no conflict because no path of hyperedges leads from $M_{C}$ to $W$ and $M_{C}$ is connected in the hypergraph with edge set $\mathfrak{S}$.

We will use the colouring $\Lambda$ to construct the graph $J_{1} \in \operatorname{Forb}(G, H)$. Choose 
two vertices $r$ and $b$ such that $\{r, b\} \cap V\left(A_{1}\right)=\varnothing$. Let $J_{1}$ be the graph with vertex set $V\left(J_{1}\right)=V\left(A_{1}\right) \cup\{r, b\}$. The edges of $J_{1}$ are such that for $J_{1} \mid V\left(A_{1}\right)=A_{1}$ the vertices $r$ and $b$ are not adjacent. The vertex $r$ is adjacent to the vertex $x \in V\left(A_{1}\right)$ if and only if $x \in N$ or $x \in V\left(A_{1}-M_{C}\right)$ and $\Lambda(x)=$ red. The vertex $b$ is adjacent to the vertex $x \in V\left(A_{1}\right)$ if and only if $\Lambda(x)=$ blue. Note that $V(W) \subseteq N$.

Claim 1. If $M$ is not a tussel which does not have any edges in the centerblock and $\beta$ is an embedding from $M$ into $A_{1}$ then at least one vertex of $V(\beta(M))$ is adjacent to $r$.

Proof of claim. Assume to the contrary that $r$ is not adjacent to any vertex in $V(\beta(M))$. This is only possible if every vertex in $V(\beta(M))$ is either blue or an element of $V(M)-N$. Note that $V(M)-N \subseteq C$.

We first consider the case that $|V(C)|=2$, say $V(C)=\{c, x\}$. Assume that $T$ is a dual block of $M$. If $\beta(T)$ does not have a vertex in common with $V(C)$ then it contains a vertex adjacent to $r$. If $\beta(T)$ has exactly one vertex in common with $V(C)$ then this vertex is $x$ because otherwise $\beta(T)$ contains a red vertex adjacent to $r$. The last possibility is that $\beta(T)=V(C)$. In any case, for every dual block $T$ of $M, \beta(T)$ contains the vertex $x$. It follows that the vertex $x$ is isolated in $\beta(M)$. Hence we can assume that $|V(C)| \geq 3$.

If $T$ is a dual block of $M$ and $\beta(T)$ does not have a vertex in common with $C$ then $\beta(T)$ contains a vertex adjacent to $r$. Hence $\beta(T)=V(C)$ or it contains exactly one vertex of $V(C)$. If $|V(\beta(T))| \geq 3$ and $\beta(T)$ contains exactly one vertex of $V(C)$ then it contains a red vertex adjacent to $r$. Hence all dual blocks $T$ of $M$ such that $\beta(T)$ contains exactly one vertex of $V(C)$ have at most two elements. Because $|V(C)| \geq 3$ it follows that $\beta(V(C))=V(C)$. We conclude that $M$ is a tussel. If $C$ contains an edge then $N \cap V(C) \neq \varnothing$ and then $r$ is adjacent to some vertex of $V(C)$ and hence to a vertex of $\beta(M)$. We arrive at the situation discussed in Case 3.

Claim 2. If $\beta$ is an embedding from $M$ into $A_{1}$ then at least one vertex of $V(\beta(M))$ is adjacent to $b$.

Proof of claim. Assume for a contradiction that $b$ is not adjacent to any vertex in $V(\beta(M))$. The vertex $b$ is adjacent to every blue vertex in $V(A)$. Hence all of the vertices in $V(\beta(M))$ are red. Let $\mathfrak{T}$ be the set of all dual blocks $T$ of $M$ such that $M \mid T \cong C$. Then for every $T \in \mathfrak{T}$ the dual block $\beta(T) \in \mathfrak{T}$ contains only red vertices and hence $V(\beta(T)) \subseteq V(M)$ and hence $V(\beta(T)) \in \mathfrak{T}$. It follows that the map $\beta$ maps injectively $\mathfrak{T}$ to $\mathfrak{T}$. This implies that there is a $T \in \mathfrak{T}$ such that $\beta(T)=V(C)$. The vertex $c \in V(C)$ is adjacent to the vertex $b$.

Proof of Lemma 13. We will assume from now on that the graphs $M$ and $A$ are not as in Case 1 or Case 2 or Case 3. The identity map embeds $A$ into the graph $J_{1}$ and $J_{1} \mid(V(M-c) \cup\{r, b\})$ is isomorphic to $D$. In order to finish the proof of Lemma 13 it is sufficient to show that $J \in \operatorname{Forb}(G, H)$. We will in the first part of the proof assume that there is an embedding from $G$ into $J$ and show that this assumption leads to a contradiction. Then in the second part we will assume that there is an embedding from $H$ into $J$ and again show that this assumption leads to a contradiction. 
Part 1. There is no embedding from $G$ into $J_{1}$.

Let us assume to the contrary that $\beta$ is an embedding from $G$ into $J_{1}$. If $\beta(u)=b$, then $\beta$ would embed $D$ into $V(B \oplus W)$ because $b$ and $r$ are not adjacent. Because $D$ contains an isolated vertex either all of $\beta(D)$ is in $W$ or in $B$. Because $W$ is too small $\beta$ embeds $D$ into $B$ and hence into $A$. Similarly we get that $\beta(u) \neq r$. Hence $\beta(u) \in V\left(A_{1}\right)$. Because no vertex of $V\left(A_{1}\right)$ is adjacent to both vertices $r$ and $b, V(\beta(D))$ contains exactly one vertex $x \in\{r, b\}$. The vertex $\beta(v) \neq x$ because $\beta$ would then embed $M$ into $A_{1}$ which is not possible because very copy of $M$ in $A_{1}$ contains at least one vertex adjacent to $x$ (Claims 1 and 2). Hence $\beta(v) \in V(B) \oplus W$. If $\beta(v) \in V(W)$ then $\beta(D) \subseteq V(W) \cup\{x\}$ which is not possible. Hence $\beta(v) \in V(B)$. Then $V(\beta(M)) \subseteq V(B) \cup\{x\} \subseteq V(A) \cup\{x\}$. We are then in a situation to apply Lemma 12 with $a=\beta(v), \delta=\beta$ and $p=\beta^{-1}(x)$.

Part 2. There is no embedding from $H$ into $J_{1}$.

Assume to the contrary that $\beta$ is an embedding from $H$ into $J_{1}$.

Let $X=V(\beta(A)) \cap V(W)$. It follows from the choice of $W_{1}$ and the construction of $J_{1}$ that $\left|V\left(W_{1}\right)\right|-1 \leq|X| \leq\left|V\left(W_{1}\right)\right|$. We first investigate the possibility that $|X|=\left|V\left(W_{1}\right)\right|-1$. It follows then that $|V(B) \cap V(\beta(A))| \geq 1$. Otherwise because $|V(A)|>|V(M)|$ we get that $V(\beta(A))=X \cup\{r, b\}$ and hence $\beta(w) \in V(B)$ because $r$ is adjacent to every vertex in $V(W)$. If $\beta(w) \in V(B)$ then $X=\varnothing$ which implies $2=|V(A)|>|V(M)|>2$. We conclude that $|V(B) \cap V(\beta(A))| \geq 1$ and hence that $\beta(w) \notin V(W)$. This in turn implies that $V(\beta(H))=X \cup V(B) \cup\{r, b\}$.

If $x \neq \varnothing$ then no vertex in $X \cup V(B)$ has degree zero in $\beta(H)$ and because $b$ is adjacent to $c$ it follows that $\beta(w)=r$. This is not possible because $r$ is adjacent to every vertex in $X$. Hence $X=\varnothing$.

Then $W_{1}=\{z\}$ for some $z \in V(W)$ and $A=B \oplus z$ and $V(\beta(H))=$ $V(B) \cup\{r, b\}$. If $\beta(w) \in\{r, b\}$ then $\beta(w)=r$ because $b$ is adjacent to $c$ and hence we obtain $A \cong J_{1} \mid(V(B) \cup\{r\})$. This is impossible because $r$ is not adjacent to all vertices in $B$ while $z$ is adjacent to all vertices of $B$. Hence $\beta(w) \in V(B)$ and $\{r, b\} \subseteq \beta(A)$. The vertex $z$ is adjacent to all vertices of $A$ and hence if $\beta(z) \in V(B)$ then $\beta(z)$ is adjacent to both vertices $r$ and $b$ but there is no such vertex in $V(B)$. It is also impossible that $\beta(z) \in\{r, b\}$ because the degree of $z$ in $A$ is $|V(B)|$ while the degree of $b$ and the degree of $r$ in $J$ is at most $|V(B)|-1$.

We are left with the possibility that $|X|=\left|V\left(W_{1}\right)\right|$. Assume that $X \neq \varnothing$. If $\beta(w) \in V(W)$ then $r \notin V(\beta(A))$ and $V(B) \cap V(\beta(A))=\varnothing$. Hence $V(\beta(A)) \subseteq\{b\} \cup V\left(W_{1}\right)$ which is not possible because $b$ is not adjacent to any vertex of $W_{1}$ and $A=B \oplus W_{1}$. Therefore $\beta(w) \notin V(W)$. It is also not possible that $\beta(w) \in V(B) \cup\{r\}$ because all those vertices are adjacent to the vertices in $X$. Hence $\beta(w)=b$ and $V(\beta(A))=X \cup V(B-c) \cup\{r\}$. This implies that there is no blue vertex in $B-c$ and hence that $M_{C}=B$. This is impossible because $|V(A)|>|V(M)|$. We conclude that $0=|X|=\left|W_{1}\right|$ and hence that $B=A$.

Let $J=J_{1} \mid(V(A) \cup\{r, b\})$. We have proven that if there is an embedding from $H$ into $J_{1}$ then there is an embedding from $H$ into $J$. It is therefore sufficient to prove that $H$ cannot be embedded into $J$. We will assume for a contradiction that $\beta$ is an embedding from $H$ into $J$. There is an embedding $\gamma$ from $M$ into $A$ and in order to simplify notation we will assume from now on that $\gamma$ is the identity map. 
Note that the graph $A$ does not contain an isolated vertex. Assume that $a$ is such an isolated vertex. Then $a \notin V(M) \subseteq V(A)$ otherwise $M$ would have an isolated vertex and if $a \in V(A)-V(M)$ then for any vertex $p \in V(M)$ the identity map is an embedding from $M-p$ into $A$ and $a$ a vertex not adjacent to any vertex in $M-p$. This would allow us to apply Lemma 12 . We conclude further that also the graph $J$ does not have an isolated vertex. Because $b$ is adjacent to $c$ and if the vertex $r$ is isolated in $J$ then $c$ would be an isolated vertex of $M$. Because $|V(J)|=|V(H)|+1$ and $w$ is an isolated vertex of $H$ it follows that $\beta(w)$ has degree at most one and because the graph $J$ does not contain an isolated vertex it follows that $\beta(w)$ has degree exactly one. In the sequel we will one after another discuss the possibilities $\beta(w)=b, \beta(w)=r$ and $\beta(w) \in V(A)$.

Possibility 1: $\beta(w)=b$.

Assume that there exists an embedding $\beta$ from $H$ into $J$ with $\beta(w)=b$. Then according to the previous paragraph the vertex $\beta(w)=b$ must have degree one. Hence in order to prove that $\beta(w) \neq b$ it is sufficient to prove that the degree of $b$ is larger than one, a fact which we will use later on again. If the degree of $b$ is one then the only vertex coloured blue by $\Delta$ is the vertex $c$. This is only possible if $V(A)=V(M)$ in contradiction to the assumption that $|V(A)|>|V(M)|$.

Possibility 2: $\beta(w)=r$.

This is a more difficult case and we will use the next six paragraphs to deal with it. Assume for a contradiction that $\beta(w)=r$. If $V(\beta(A))=V(A)$, then the vertex $r \in V(J)$ would be isolated and therefore $c$ would be isolated in $M$. Hence $V(\beta(A))=V(A-d) \cup\{b\}$ for some vertex $d \in V(M)$ adjacent to $r$. The vertex $r$ is adjacent to the vertex $d$ but to no other vertex of $A$. The vertex $c$ is then adjacent to the vertex $d$ and to no other vertex of $M$. Hence every vertex $x$ of a dual block $C_{1}$ of $M$ with $\left|V\left(C_{1}\right)\right|=|V(C)|$ has degree one in $M$. This follows from the choice of $C$ and $c$ and the fact that $M$ does not contain an isolated vertex.

If $M$ is a tussel then $c$ is adjacent to the vertex $d \in V(C)$. (Otherwise $C$ would not contain an edge and we would be in the situation of Case 3.) Hence every other dual block $S$ of $M$ contains $c$. Because $c$ is blue the other vertices of $S$ are red and adjacent or $r$. This implies that $M=C$, all other elements of $\mathfrak{S}$ have size at most two and contain exactly one vertex in $M-c$.

In this situation define the graph $I$ as follows. The vertex set of $I$ is $V(A) \cup$ $\{b\}$. The edges of $I$ are such that $I \mid V(A)=A$ and $V(A)-V(M)$ is the set of vertices adjacent to $b$. The identity map embeds $A$ into $I$ and $I \mid(V(M) \cup\{b\})$ is isomorphic to $D$. The graph $G$ cannot be embedded into $I$, for assume that $\alpha$ is an embedding from $G$ into $I$. If $\alpha(u)=b$ then $D$ can be embedded into $A$. If $\alpha(u) \in M$ the $\alpha(u)$ not adjacent to $b$ and hence $D$ can be embedded into $A$. Hence $\alpha(u) \in V(A)-V(M)$. If $\alpha(v)=b$ then there are only $V(M)-1$ vertices adjacent to $\alpha(u)$ and not to $\alpha(v)$. If $\alpha(v) \in V(A)$ we can apply Lemma 12. If $H$ is embedded into $I$ then it is isomorphic to $I$ which is impossible because $H$ has fewer edges than $I$.

Next we consider the case that $M$ is not a tussel. If $d \notin V(C)$, then $d \in V(S)$ for some dual block $S \neq C$ of $M$. If $c \in V(S)$, then of course $V(C) \cap V(S)=\{c\}$, and we choose a vertex $x \neq c$ with $x \in V(C)$. The 
vertex $x \notin S$ and because $\bar{S}$ is two-connected it contains at least two more vertices besides $c$ and $d$ as the vertices $c$ and $d$ are adjacent. The vertex $x$ is adjacent to all the vertices of $S-c$. Because the degree in $M$ of any vertex of $C$ is one we obtain that $c \notin V(S)$ holds. If then $V(C) \cap V(S) \neq \varnothing$, assume that $\{y\}=V(C) \cap V(S)$. The vertex $c$ is adjacent to every vertex of $S-y$ and hence $V(S)=\{y, d\}$. Hence, every dual block of $M$ which is not equal to $S$ contains the vertex $c$, otherwise $c$ would be adjacent to yet another vertex different from $d$. Further, there is at most one other dual block $T$ of $M$ besides $S$ and $C$, and $T$ contains exactly two vertices, namely $c$ and one other vertex, say $z$. If $T$ would contain one more vertex besides $c$ and $z$ the vertex $y$ would have at least degree two in $M$ and because $y \in V(C)$ this would be in contradiction to the choice of the vertex $c$. For just this same reason there does not exist another block of $M$ different from $T$ containing $c$. Note that $z \neq d$, otherwise the set $\{z=d, c, y\}$ would be part of a dual block intersecting $C$ in two vertices. If $C$ contains a third vertex $x$ and $c \neq x \neq y$, then $x$ would be adjacent to $d$ and $z$. Hence $|V(C)|=2$. This implies then that $z$ is adjacent to $d$ and $y$ and contained in a dual block of $M$ with a maximal number of vertices which means that $S$ and $C$ are the only two dual blocks of $M$. No vertex $x \in V(C)-y$ is adjacent to $y$, because $x$ is already adjacent to $d$. The vertex $y$ is not adjacent to $d$ because $S$ is a dual block of $M$. Hence $y$ is an isolated vertex of $M$.

We are left with the possibility that $V(C) \cap V(S)=\varnothing$ and hence $V(S)=\{d\}$ because if $V(C) \cap V(S)=\varnothing$ every vertex of $C$ is adjacent to every vertex of $S$. Then $d$ is an isolated vertex of $\bar{M}$, the graph $M$ contains only the two dual blocks $S$ and $C$ and no two vertices of $C$ are adjacent. For every $S \in \mathfrak{S}$ with $|S| \geq 2$, there is at most one vertex $x \in S$ with $\Delta(x)=$ blue. Hence if $|V(C)|>2, S \in \mathfrak{S}$ and $|S|=|V(C)|$, then $S=V(C)$. If $|V(C)|=2$, every element of $S$ consists of a pair of nonadjacent vertices. The graph $M$ consists of three vertices, one of them adjacent to the other two which are not adjacent. Denote one of the two vertices of $M$ which are not isolated in $\bar{M}$ by $p$. If $S, T \in \mathfrak{S}$ with $S \cap T \neq \varnothing$, say $S \cap T=\{a\}$, then $M-p$ is embedded into $A$ in such a way that $a$ is not adjacent to any vertex of the image. Then we apply Lemma 12 . Hence no two different elements of $\mathfrak{S}$ have a vertex in common. Because $r$ is only adjacent to the vertex $d$ of $A, \mathfrak{S}$ has then only one element, namely $C$. Then $d$ is a vertex of $A$ which is adjacent to the unique set of $|V(C)|=2$ pairwise not adjacent vertices of $A$. If $J \mid(V(A-d) \cup\{b\})$ is isomorphic to $A$, the vertex $b$ must be adjacent to a set of $|V(C)|=2$ pairwise not adjacent vertices. Otherwise $J \mid(V(A-d) \cup\{b\})$ would contain fewer vertices which are adjacent to a set of two pairwise not adjacent vertices than $A$.

Because $b$ is not adjacent to every vertex of $C$, it follows finally that $d \in$ $V(C)$ and hence every dual block of $M$ contains $c$. Then if $y \in V(M)-V(C)$, $d$ would be adjacent to $y$ and to $c$. Hence $M=C$. This implies that every vertex of $M$ has degree one, the number $|V(M)|$ is even and at least four. If $S \in \mathfrak{S}$ with $|S|=|V(M)|$ and $S \neq V(C)$ then there is at least one vertex $x \in S-V(C)$ with $\Delta(x)=$ red and hence adjacent to $r$. We conclude that there is no $S \in \mathfrak{S}$ with $|S|=|V(M)|$ and $S \neq V(C)$. But this is the situation dealt with in Case 1. 
Possibility 3: $\beta(w) \in V(A)$.

Remember that $\beta(w)$ has degree one in $J$. If $\beta(w)$ is adjacent to $r$ or $b$ then $\beta(w)$ would be an isolated vertex of $A$. That $A$ has an isolated vertex is a situation which we have already discarded. Hence $\beta(w)$ is adjacent to some vertex $d$ of $A$ and then $\beta(w)$ is not adjacent to $r$ or $b$. Hence $\beta(w) \in V(C-c)-N$. If the vertex $d \notin V(M)$ then the vertex $\beta(w)$ would be isolated in $M$. Hence both vertices $\beta(w)$ and $d$ are vertices of $M$ and $V(\beta(A))=V(A-\beta(w)-d) \cup\{r, b\}$ and hence $A \cong J \mid(V(A-\beta(w)-d) \cup\{r, b\})$.

We will prove in this paragraph that $\{\beta(w), d\} \subseteq V(C)$. For if not then $\overline{C-\beta(w)-d}$ is connected because $\bar{C}$ is two-connected. In $C$, The vertex $b$ is only adjacent to $c$ and $r$ is not adjacent to $c$ and not to $b$. Hence $\beta(A) \mid(V(C-\beta(w)-d) \cup\{r, b\})$ is a dual block of $\beta(A)$ with more vertices than $C$, in contradiction to being an $M$-tree.

Because the vertex $\beta(w)$ is only adjacent to $d$, every vertex of $A$ except possibly $d$ is in a dual block which also contains $\beta(w)$. Hence we are in the situation that every dual block of $A$ contains the vertex $\beta(w)$, the set $\{\beta(w), d\} \subseteq V(C)$ and $\beta(w)$ is not adjacent to $c$. For the remainder of the proof we will investigate the value of $\beta(\beta(w))$, that is whether it is possible that $\beta(\beta(w))=b$ or that $\beta(\beta(w))=r$ or that $\beta(\beta(w)) \in V(A)$. Note that the degree of $\beta(\beta(w))$ in $\beta(A)=J \mid((V(A)-d-\beta(w)) \cup\{r, b\})$ is one.

Possibility 3.1: $\beta(w) \in V(A)$ and $\beta(\beta(w))=b$.

If $\beta(\beta(w))=b$ then because $b$ is not adjacent to $\beta(w)$ or $d$, the valence of $b$ in $J$ is the same as the valence of $b$ in $\beta(A)$. The valence of $\beta(\beta(w))$ in $\beta(A)$ is one and hence the valence of $b$ in $J$ is one, a situation which we discussed earlier.

Possibility 3.2: $\beta(w) \in V(A)$ and $\beta(\beta(w)) \in r$.

Assume first that $r$ is not adjacent to $d$. Then because $r$ is not adjacent to $\beta(w)$ as $c$ is not adjacent to $\beta(w)$, the degree of $r$ in $J$ is the same as the degree of $r$ in $\beta(A)$. Hence the degree of $r$ in $J$ is one. Because $c$ is not isolated in $M$ the vertex $r$ is adjacent to the unique neighbour of $c$ in $M$. Because $d$ has degree one in $C$ there is no vertex $x \in V(M)-V(C)$ otherwise $d$ would have to be adjacent with this vertex $x$ (they are not in a common dual block of $A$ ), and hence have valence at least two in $M$ in contradiction to the choice of $c$ (in both cases $M$ a tussel and $M$ not a tussel). This implies that $C=M$ consists of a set of pairwise disjoint edges together with a set of isolated vertices. Every dual block $S$ of $A$ different from $C$ has at most two vertices, otherwise one of its vertices different from $\beta(w)$ would be coloured red and hence be adjacent to $r$ which then would have a larger degree than one in $\beta(A)$. Hence we arrive at a situation discussed in Case 2.

We consider next the case that $\beta(\beta(w))=r$ and that $r$ is adjacent to $d$. Then because $r$ is not adjacent to $\beta(w)$ and the degree of $\beta(\beta(w))$ is one in $\beta(A)$, the degree of $r$ in $J$ is two, it is adjacent to $d$ and to one other vertex, say $y$. Because $r$ is adjacent to $d$ also $c$ is adjacent to $d$. Hence $d$ has degree at least two in $C$. There is no vertex $x \in V(M)-V(C)$ because $d$ would have to be adjacent with this vertex $x$ (they are not in a common dual block of $A$ ), and hence have valence at least three in $M$. Due to the choice of $c$ also $c$ and hence $r$ would be adjacent to at least three vertices of $M$ (again in both cases). This would imply that $r$ has at least degree two in $\beta(A)$. Hence 
$C=M$. Every dual block $S$ of $A$ different from $C$ has at most two vertices, otherwise one of its vertices different from $\beta(w)$ would be coloured red and therefore be adjacent to $r$ which then would have a larger degree than two in $\beta(A)$. Hence we arrive at a situation discussed in Case 2 .

Possibility 3.3: $\beta(w) \in V(A)$ and $\beta(\beta(w)) \in V(A)$.

The graph $\beta(A)=J \mid((V(A)-\beta(w)-d) \cup\{r, b\})$ and hence if a vertex $x$ in $V(A)-\beta(w)-d$ has degree at least three in $J$ then $\beta(\beta(w)) \neq x$. Every vertex $x \in V(A)-V(C)$ is adjacent to $b$ or to $r$ and is also adjacent to $c$ and to $d$. Hence $\beta(\beta(w)) \in V(C)-\beta(w)-d$. It follows then that $\beta(\beta(w))$ is adjacent to every vertex $x \in V(A)-V(C)$ and because $V(A)-V(C) \subseteq V(\beta(A))$ and the degree of $\beta(\beta(w))$ is one that there is exactly one vertex in the set $V(A)-V(C)$. (There is at least such a vertex because $|V(M)|<|V(A)|$.) This implies that we are again in a situation already discussed in Case 2.

\subsection{The CASE IN WHICH $M$ IS A COMPLETE GRAPH}

Lemma 14. Let $M$ be a complete graph on $m \geq 3$ vertices and $A$ the complete graph on a vertices with $m+1 \leq a \leq 2 m-2$. For $u, v$, and $w$, three vertices not in $A$ or $M$, the graphs $D, H$, and $G$ are given by $D=v+M, G=u \oplus D$, and $H=w+A$. Then there exists a graph $J \in \operatorname{Forb}(G, H)$ such that both graphs $A$ and $D$ can be embedded into $J$.

Proof. Let $L \subseteq V(A)$ with $|L|=m-1$ and $r, b$ two vertices with $\{r, b\} \nsubseteq$ $V(A)$. The graph $J$ has as vertex set the set $V(J)=V(A) \cup\{r, b\}$. The vertices of $J$ are such that $J \mid V(A)=A$ and $r$ are $b$ are not adjacent. The vertex $x \in V(A)$ is adjacent to $r$ if and only if $x \in L$, and $x \in V(A)$ is adjacent to $b$ if and only if $x \in V(A)-L$. Clearly $A$ can be embedded into $J$ and $J \mid(L \cup\{r, b\})$ is isomorphic to $D$.

We prove that $J \in \operatorname{Forb}(G)$. Assume to the contrary that there is an embedding $\alpha$ from $G$ into $J$. The vertex $\alpha(v) \notin V(A)$ because to every vertex $y$ of $A$ there is only one vertex of $J$ not adjacent to $y$, but $M$ contains at least three vertices and no vertex of $M$ is adjacent to $v$. Assume that $\alpha(v)=r$. Then $V(\alpha(M)) \subseteq V(A-L) \cup\{b\}$. But $|V(A-L) \cup\{b\}| \leq 2 m-2-(m-1)+1=m$. Hence $V(\alpha(M))=V(A-L) \cup\{b\}$, which implies that $\alpha(u)$ is adjacent to both vertices $r$ and $b$. This is a contradiction because there is no vertex in the graph $J$ adjacent to both vertices $r$ and $b$. If $\alpha(v)=b$, then $V(\alpha(M)) \subseteq L \cup\{r\}$. But $|L \cup\{r\}|=m$. Hence $V(\alpha(M))=L \cup\{r\}$, which implies that $\alpha(u)$ is adjacent to both vertices $r$ and $b$. This is a contradiction because there is no vertex in the graph $J$ adjacent to both vertices $r$ and $b$. Hence $J \in \operatorname{Forb}(G)$.

We prove next that $J \in \operatorname{Forb}(H) .|V(J)|=V(H)+1=a+2$ and $w \in V(H)$ is not adjacent to $a$ vertices of $H$. Hence, the degree of $\alpha(w)$ in $J$ is at most one. The $J$-degree of a vertex $x \in V(A)$ is $2 m-3+1>1$, the $J$-degree of $r$ is $m-1>1$ and the degree of $b$ in $J$ is $a-(m-1) \geq m+1-m(m-1)=2$. Hence $J \in \operatorname{Forb}(G)$.

Lemma 15. Let $M$ be the complete graph on $m \geq 2$ vertices and $A$ the complete graph on $a \geq 2 m$ vertices. For $u, v$, and $w$, three vertices not in $A$ or $M$, the graphs $D, H$, and $G$ are given by $D=v+M, G=u \oplus D$ and $H=w+A$. Then the family $\operatorname{Forb}(G, H)$ of graphs does not have the Ramsey property.

Proof. We first prove the following statement: If $T$ is a graph in $\operatorname{Forb}(G, H)$ 
and $C$ is a complete subgraph of $T$ with $|V(C)| \geq 2 m-1$ then every vertex in the connected component of $T$ which contains $C$ is adjacent to at least $|V(C)|-(m-1)$ vertices of $C$.

Let $T \in \operatorname{Forb}(G, H)$ and $C$ be a complete subgraph of $T$ with $|V(C)| \geq$ $2 m-1$. Let $x \in V(T-C)$ be adjacent to the vertex $z \in V(C)$, and $y \in V(T)$ be adjacent to $x$. If $L \subseteq V(C-z)$ with $|L|=m$, then $T \mid(L \cup\{z, x\})$ is isomorphic to $G$ unless $x$ is adjacent to at least one vertex of $L$. Hence $x$ is not adjacent to at most $m-1$ vertices of $C$. Let $S=\{c \in V(C): c$ is adjacent to $x\}$. Then $|S| \geq 2 m-1-(m-1)=m$. Let $R \subseteq S$ with $|R|=m$. Then $T \mid(R \cup\{x, y\})$ is isomorphic to $G$ unless $y$ is adjacent to some vertex in $R$. Hence $y$ must be adjacent to at least $|V(C)|-(m-1)$ vertices of $C$. We conclude that every vertex in the connected component of $T$ which contains $C$ is adjacent to at least $|V(C)|-(m-1)$ vertices of $C$.

Let $s=a(m-1), r=\left(\begin{array}{c}s \\ m-1\end{array}\right)$ and $U$ be the graph $U=K_{a-1}+K_{a-1}$ with $U_{1}$ and $U_{2}$ the two-connected components of $U$. Clearly $U \in \operatorname{Forb}(G, H)$ holds. We will prove that there is no graph $T \in \operatorname{Forb}(G, H)$ with $T \rightarrow(U)_{r+s}^{1}$. Assume to the contrary that there is such a graph $T \in \operatorname{Forb}(G, H)$ with $T \rightarrow$ $(U)_{r+s}^{1}$. We will assume first that $T$ contains a complete subgraph $C$ with $|V(C)| \geq a(m-1)+1$. Clearly then because $H$ cannot be embedded into $T$, $T$ is connected. Hence every vertex of $T$ is adjacent to at least $|V(C)|-(m-1)$ vertices of $C$. But then, if $S \subseteq V(T-C)$ and $|S| \leq a$, there is at least one vertex in $V(C)$ which is adjacent to all the vertices of $S$. We colour now the vertices of $T$ with two colours. All vertices of $V(C)$ are coloured red and all vertices of $V(T-C)$ are coloured blue. Clearly, $U$ cannot be embedded into $C$. If $\alpha$ is an emledding from $U$ into $V(T-C)$ let $x \in V\left(\alpha\left(U_{2}\right)\right)$ and $S=V\left(\alpha\left(U_{1}\right)\right) \cup\{x\} .|S|=a$ and hence there is a vertex $z \in V(C)$ which is adjacent to all vertices of $S$. This is a contradiction because $T \mid\left(V\left(U_{1}\right) \cup\{z, x\}\right)$ contains a subgraph which is isomorphic to $G$. Hence $T \nrightarrow(U)_{2}^{1}$. The graph $T$ does therefore not contain a complete subgraph $C$ with $|V(C)| \geq a(m-1)+1$.

Let $T_{0}, T_{1}, \ldots, T_{l-1}$ be the connected components of $T$. For $i \in l$ let $C_{i}$ be a maximal complete subgraph of $T_{i}$. If $\left|V\left(C_{i}\right)\right| \geq 2 m-1$ we let $\gamma_{i}$ be an injection from the $\left(\left|V\left(C_{i}\right)\right|-(m-1)\right)$-subsets of $V\left(C_{i}\right)$ to $r$. The embedding $\delta: V(T) \rightarrow r+s$ is a colouring of the vertices of $T$ with $r$ colours given as follows. If for some $i \in l,\left|V\left(C_{i}\right)\right|<2 m-1$ we colour the vertices of $T_{i}$ with the number 0 . If for $i \in l,\left|V\left(C_{i}\right)\right| \geq 2 m-1$ we associate with each vertex $x \in V\left(T_{i}-C_{i}\right)$ a subset $S_{x}$ of $V\left(C_{i}\right)$ with $\left|S_{x}\right|=\left|V\left(C_{i}\right)\right|-(m-1)$ and such that every vertex of $S_{x}$ is adjacent to $x$. Then we put $\delta(x)=\gamma_{i}\left(S_{x}\right) . \delta \mid V\left(C_{i}\right)$ is an injection from $V\left(C_{i}\right)$ into the numbers from $r$ to $r+s-1$. We will have proven that $T \nrightarrow(U)_{r+s}^{1}$ if for each $j \in r+s, U$ cannot be embedded into $\delta^{-1}(j)$. If $r \leq j \leq r+s-1, \delta^{-1}(j)$ consists of a set of pairwise not adjacent vertices. Assume that for some $j \in r, \delta^{-1}(j)$ contains a complete graph $W$ on $a-1$ vertices. Let $W$ be a subgraph of the connected component $T_{i}$. Clearly then $\left|V\left(C_{i}\right)\right| \geq 2 m-1$ because $a \geq 2 m$ by assumption. For all pairs $x, y$ of vertices in $V(W), S_{x}=S_{y}$. Let $S=S_{x}$ for some $x \in V(W)$. Then $T \mid(V(W) \cup S)$ is a complete graph with $|V(W) \cup S|=a-1+\left|V\left(C_{i}\right)\right|-(m-1)>\left|V\left(C_{i}\right)\right|$ vertices. Because $C_{i}$ is the largest complete subgraph of $T_{i}$, no complete subgraph with $a-1$ vertices can be embedded into $\delta^{-1}(j)$. Hence $U$ cannot be embedded into $\delta^{-1}(j)$. 
Lemma 16. Let $M$ be the complete graph on $m \geq 4$ vertices and $A$ the complete graph on a vertices with $a=2 m-1$. For $u, v$, and $w$ three vertices not in $A$ or $M$, the graphs $D, H$, and $G$ are given by $D=v+M, G=u \oplus D$, and $H=w+A$. Then there exists a graph $J \in \operatorname{Forb}(G, H)$ such that both graphs $A$ and $D$ can be embedded into $J$.

Proof. Let $V(A)=\left\{y_{i}: i \in m-1\right\} \cup\left\{z_{i}: i \in m-1\right\} \cup\{b\}$ and $V(M)=$ $\left\{x_{i}: i \in m\right\}$. We assume that $V(A) \cap V(M)=\varnothing$. The graph $J$ will have vertex set $V(J)=V(A) \cup V(M)$. The edges of $J$ are such that $J \mid V(A)=A$ and $J \mid V(M)=M$. For $i \in m-1$ the vertex $x_{i}$ is adjacent to $p \in V(A)$ if and only if $p \in V\left(A-b-y_{i}-z_{i}\right)$. The vertex $x_{m-1}$ is adjacent to $p \in V(A)$ if and only if $p \in V(A-b)$. Note that $A$ can be embedded into $J$ and $J \mid(V(M) \cup\{b\})$ is isomorphic to $D$. Assume that there is an embedding $\alpha$ from $G$ into $J$. Then $\alpha(v) \notin V(A-b)$ because there is then only one vertex not adjacent to $\alpha(v)$ while $v$ is not adjacent to at least four vertices. If $\alpha(v) \in V(M)$, then there are at most three vertices in $J$ not adjacent to $\alpha(v)$. If $\alpha(v)=b$, then $\alpha(M)=M$ because $b$ is adjacent to every vertex of $A-b$. But then $\alpha(u) \in V(A-b)$ and no vertex in $V(A-b)$ is adjacent to all vertices of $M$. Hence $J \in \operatorname{Forb}(G)$. Assume next that there is an embedding $\alpha$ from $H$ into $J .|V(J)|=|V(H)|+m-1$ and $w$ is an isolated vertex of $H$. Hence $\alpha(w)$ has at most degree $m-1$ in $J$. Every vertex of $J$ has at least degree $m$. Hence $J \in \operatorname{Forb}(G, H)$.

Lemma 17. Let $M$ be the complete graph on $m=3$ vertices and $A$ the complete graph on $a=2 m-1=5$ vertices. For $u, v$, and $w$, three vertices not in $A$ or $M$, the graphs $D, H$, and $G$ are given by $D=v+M, G=u \oplus D$, and $H=w+A$. Then the family $\operatorname{Forb}(G, H)$ of graphs does not have the Ramsey property or there exists a graph $J \in \operatorname{Forb}(G, H)$ such that both graphs $A$ and $D$ can be embedded into $J$.

Proof. Let $R$ be the graph with vertex set $V(R)=C \cup\{m, e, d\}$ where $C=$ $\left\{c_{i}: i \in 6\right\}$. In $R$, the vertex $m$ is adjacent to the vertex $x$, if and only if $m \in C$. For $i>j, c_{i}$ is adjacent to $c_{j}$ if and only if $i-j \equiv 1 \bmod 6$ or if $i=4$ and $j=1$ or if $i=5$ and $j=2$. The vertex $e$ is adjacent to the vertex $c_{i}$ if and only if $i \in\{0,1,3,5\}$. The vertex $d$ is adjacent to the vertex $c_{i}$ if and only if $i \in\{0,2,3,4\}$. The vertices $e$ and $d$ are adjacent. We claim that the graph $R$ has the following four properties:

P1: $R \in \operatorname{Forb}\left(K_{4}\right)$.

P2: $R \in \operatorname{Forb}\left(K_{3}+K_{1}\right)=\operatorname{Forb}(D)$.

P3: If $S \subseteq V(R)$ has the property that for all embeddings $\alpha$ from $K_{3}$ into $R, S \cap V\left(\alpha\left(K_{3}\right)\right) \neq \varnothing$, then $S$ contains two adjacent vertices.

P4: The graph $R$ has 23 degrees.

Note first that $R \mid C$ does not contain a triangle. Hence, the vertex $m$ is not contained in any complete graph on four vertices. Similarly $e$ and $d$ are not contained in any complete graph on four vertices unless they are together in the same complete graph on four vertices. But the set of vertices adjacent to both vertices $e$ and $d$ is $\left\{c_{0}, c_{3}\right\}$ and the vertex $c_{0}$ is not adjacent to the vertex $c_{3}$. This proves $\mathrm{P} 1$. Let for $x \in V(R), N(x)=\{y: y \neq x\}$ and $y$ is not adjacent to $x$. In order to establish $\mathrm{P} 2$ we have to prove that for all vertices $x$ of $R, R \mid N(x)$ does not contain a triangle. $|N(m)|=2$ and for all other vertices of $R,|N(x)|=3$. We verify by vertex by vertex inspection 
that for every vertex $x \in V(R-m), N(x)$ contains two nonadjacent vertices. In order to establish $\mathrm{P} 3$, we have to prove that for every maximal independent subset $S$ of $V(R)$ there is a triangle in $R$ which has no vertex in $S$. If $m \in S$ then either $e$ or $d$ are in $S$ but not both. If $e \in S, R \mid\left\{d, c_{3}, c_{2}\right\}$ is a triangle disjoined from $S$. If $d \in S, R \mid\left\{e, c_{0}, c_{1}\right\}$ is a triangle disjointed from $S$. Hence $m \notin S$. If $e \in S$, then $d \notin S$, hence $S=\left\{e, c_{2}, c_{4}\right\}$. Then $R \mid\left\{c_{0}, c_{1}, m\right\}$ is a triangle disjoined from $S$. If $d \in S$, then $e \notin S$, hence $S=\left\{d, c_{1}, c_{5}\right\}$. Then $R \mid\left\{c_{2}, c_{3}, m\right\}$ is a triangle disjoined from $S$. This leaves $S \subseteq C$. If $S=\left\{c_{0}, c_{3}\right\}$, then $R \mid\left\{c_{1}, c_{2}, m\right\}$ is a triangle disjoined from $S$. Hence $S$ does not contain both vertices $c_{0}$ and $c_{3}$. Therefore, if $c_{0} \in S$, then $S=\left\{c_{0}, c_{4}, c_{2}\right\}$ and $R \mid\left\{e, d, c_{3}\right\}$ is a triangle disjoined from $S$. If $c_{3} \in S$, then $S=\left\{c_{3}, c_{1}, c_{5}\right\}$ and $R \mid\left\{e, d, c_{0}\right\}$ is a triangle disjoined from $S$. Hence neither $c_{0}$ nor $c_{3}$ is in $S$. But the set $\left\{c_{1}, c_{2}, c_{4}, c_{5}\right\}$ of vertices does not contain a maximal independent set of vertices of $R$ as a subset.

Let $T$ be the graph $R \oplus K_{1}$ and denote the vertex of $T$ adjacent to all the other vertices of $T$ by $z$. Note that $T \in \operatorname{Forb}\left(G, K_{5}\right)$. Clearly $T \in \operatorname{Forb}\left(K_{5}\right)$ because $R \in \operatorname{Forb}\left(K_{4}\right)$. If $\alpha$ is an embedding from $G$ into $T$, then $z \in$ $V(\alpha(G))$ because $R \in \operatorname{Forb}\left(K_{4}\right)$. Then $\alpha(u)=z$ because $G$ contains only one vertex adjacent to all the other vertices of $G$. This implies that $V(\alpha(D)) \subseteq V(R)$ in contradiction to property $\mathbf{P} 2$ of $R$. Let $L_{1}$ be a graph which does not contain a triangle and has chromatic number 24 . Let $L$ be the graph $L_{1} \oplus K_{1}$ and denote the vertex of $L$ adjacent to all the other vertices of $L$ by $y$. Assume also that $V(L) \cup V(T)=\varnothing$. Note that $L \in \operatorname{Forb}\left(G, K_{5}\right)$ because $L$ does not contain a compLete graph on four vertices.

Let $N$ be the graph $N=T+L+D$. Clearly then $N \in \operatorname{Forb}\left(G, K_{5}\right)$ and hence $N \in \operatorname{Forb}(G, H)$. Let us assume that $\operatorname{Forb}(G, H)$ has the Ramsey property. It follows then from Theorem 5 that $N$ can be disjointly amalgamated with $N$ on the pair $z$ and $y$ of vertices to yield the graph $J$. In doing so the graph $T$ will be disjointly amalgamated with $L$ on the pair $z$ and $y$ of vertices into a graph $B \in \operatorname{Forb}(G, H)$. We claim now that $B$ contains the graph $K_{5} \cong A$ as a subgraph. If $Q \subseteq V(R)$ with $R \mid Q \cong K_{3}$ and $x$ is a vertex of $L_{1}$, then $B \mid(Q \cup\{z=y, x\}) \cong G$ unless $x$ is adjacent to some vertex of $Q$. This means that for every vertex $x \in L_{1}$ the set $S_{x} \subseteq V(R)$ of vertices of $R$ adjacent to $x$ has a nonempty intersection with the vertices of every triangle of $R$. Hence it follows from property P3 that for every vertex $x \in L_{1}$ there are two adjacent vertices $x_{1}$ and $x_{2}$ of $R$ which are adjacent to $x$. The chromatic number of $L_{1}$ is 24 and $R$ has only 23 edges. Hence there are two adjacent vertices $k$ and $l$ of $L_{1}$ such that $\left\{k_{1}, k_{2}\right\}=\left\{l_{1}, l_{2}\right\}$. Then $B \mid\left\{z=y, k_{1}, k_{2}, k, l\right\} \cong K_{5}$. By construction the graph $D$ can be embedded into the graph $J$. Then $A$ and $D$ can both be embedded into the graph $J \in \operatorname{Forb}(G, H)$.

Lemma 18. Let $M \cong K_{1}$ and $A$ be a graph. For $u, v$, and $w$ three vertices not in $A$ or $M$, the graphs $D, H$, and $G$ are given by $D=v+M, G=u \oplus D$, and $H=w+A$. Then the family $\operatorname{Forb}(G, H)$ of graphs has the Ramsey property if and only if $A \cong K_{2}$.

Proof. If $a=|V(A)|=1$ then $H$ can be embedded into $G$ and hence we assume that $a=|V(A)| \geq 2$. If $A$ is not a complete graph then $D$ can be embedded into $A$. We assume now that $A$ is complete. Observe that if the 
graph $G \cong P_{2}$ cannot be embedded into a graph $R$, then the adjacency relation on $R$ is transitive. Hence $R$ is the sum (+) of complete graphs. If also $H$ cannot be embedded into $R$ and $R$ is not a complete graph, then the complete subgraphs of $R$ contain at most $|V(A)|-1$ vertices. Hence if $a=|V(A)| \geq 3$, then there is no graph $B \in \operatorname{Forb}(G, H)$ such that $B \rightarrow\left(K_{a-1}+K_{a-1}\right)_{2}^{1}$. For $\operatorname{Forb}(G, H)$ is then not Ramsey. If $a=2$ then $H \cong \bar{G} \cong \overline{P_{2}}$. Hence the elements of $\operatorname{Forb}(G, H)$ are then either complete graphs or complements of complete graphs. $\operatorname{Forb}(G, H)$ then has the Ramsey property.

\section{REFERENCES}

1. J. Nešetril and V. Rödl, Partitions of vertices, Comment. Math. Univ. Carolin. 17 (1976), 675-681.

2. F. Harary, Graph theory, Addison-Wesley Ser. in Math., Addison-Wesley, 1969.

3. B. Bollobás, Graph theory, An Introductory Course, Springer-Verlag, 1979.

4. V. Rödl and N. Sauer, The Ramsey property for families of graphs which exclude a given graph, Canad. J. Math. 44 (1992), 1050-1060.

5. V. Rödl, N. Sauer, and X. Zhu, Ramsey families which exclude a graph, Yellow series, no. 708, Dept. of Math., Univ of Calgary.

6. N. Sauer and X. Zhu, Graphs which do not embed a given graph and the Ramsey property, Sets, Graphs and Numbers, Colloq. Math. Soc. János Bolyai, vol. 60, North-Holland, 1991, pp. 631-636.

7. P. Erdös and A. Hajnal, On chromatic number of graphs and set systems, Acta Math. Acad. Sci. Hungar. 17 (1966), 61-99.

8. R. Fraïssé, Theory of relations, North-Holland, 1986.

9. J. Nešetril and V. Rödl, Partitions of finite relational and set systems, J. Combin. Theory Ser. A 22 (1977), 289-312.

10. A Gyárfás, On Ramsey covering numbers, Infinite and Finite Sets, Colloq. Math. Soc. János Bolyai, vol. 10, North-Holland, 1975, pp. 801-816.

11. D. P. Sumner, Subtrees of a graph and chromatic number, The Theory of Applications of Graphs (Gary Chartrand, ed.), Wiley, 1981, pp. 557-576.

12. A Gyárfás, Problems from the world surrounding perfect graphs, Zastos. Mat. 19 (1985), 413-441.

13. H. Kierstead and S. Penrice, Recent results on a conjecture of Gyárfás, preprint.

14. __ Radius two trees specify $\chi$-bounded classes, J. Graph Theory (to appear).

15. N. Sauer, Vertex partition problems, Combinatorics, Paul Erdős is Eighty, Vol. I, Kesthely, Hungary, 1993, pp. 361-377.

Department of Mathematics, University of Calgary, Calgary, Alberta, Canada T2N

E-mail address: nsauer@acs.ucalgary.ca 